\title{
Dynamical Mean-Field Theory for Markovian Open Quantum Many-Body Systems
}

\author{
Orazio Scarlatella $\odot,{ }^{1,2,3, *}$ Aashish A. Clerk, ${ }^{4}$ Rosario Fazio, ${ }^{5, *}$ and Marco Schiró ${ }^{1, \dagger, 8}$ \\ ${ }^{1}$ JEIP, USR 3573 CNRS, Collége de France, PSL Research University, \\ 11, place Marcelin Berthelot, 75231 Paris Cedex 05, France \\ ${ }^{2}$ Clarendon Laboratory, University of Oxford, Parks Road, Oxford OX1 3PU, United Kingdom \\ ${ }^{3}$ Institut de Physique Théorique, Université Paris Saclay, CNRS, CEA, F-91191 Gif-sur-Yvette, France \\ ${ }^{4}$ Pritzker School of Molecular Engineering, University of Chicago, \\ 5640 South Ellis Avenue, Chicago, Illinois 60637, USA \\ ${ }^{5}$ The Abdus Salam International Centre for Theoretical Physics, Strada Costiera 11, I-34151 Trieste, Italy
}

(Received 5 August 2020; revised 5 April 2021; accepted 17 May 2021; published 22 July 2021; corrected 16 September 2021)

A number of experimental platforms relevant for quantum simulations, ranging from arrays of superconducting circuits hosting correlated states of light to ultracold atoms in optical lattices in the presence of controlled dissipative processes, are described as open quantum many-body systems. Their theoretical understanding is hampered by the exponential scaling of their Hilbert space and by their intrinsic nonequilibrium nature, limiting the applicability of many traditional approaches. In this work, we extend the nonequilibrium bosonic dynamical mean-field theory (DMFT) to Markovian open quantum systems. Within DMFT, a Lindblad master equation describing a lattice of dissipative bosonic particles is mapped onto an impurity problem describing a single site embedded in its Markovian environment and coupled to a self-consistent field and to a non-Markovian bath, where the latter accounts for fluctuations beyond Gutzwiller mean-field theory due to the finite lattice connectivity. We develop a nonperturbative approach to solve this bosonic impurity problem, which dresses the impurity, featuring Markovian dissipative channels, with the non-Markovian bath, in a self-consistent scheme based on a resummation of noncrossing diagrams. As a first application of our approach, we address the steady state of a drivendissipative Bose-Hubbard model with two-body losses and incoherent pump. We show that DMFT captures hopping-induced dissipative processes, completely missed in Gutzwiller mean-field theory, which crucially determine the properties of the normal phase, including the redistribution of steady-state populations, the suppression of local gain, and the emergence of a stationary quantum-Zeno regime. We argue that these processes compete with coherent hopping to determine the phase transition toward a nonequilibrium superfluid, leading to a strong renormalization of the phase boundary at finite connectivity. We show that this transition occurs as a finite-frequency instability, leading to an oscillating-in-time order parameter, that we connect with a quantum many-body synchronization transition of an array of quantum van der Pol oscillators.

DOI: 10.1103/PhysRevX.11.031018

\footnotetext{
* On leave from Dipartimento di Fisica, Università di Napoli "Federico II," Monte S. Angelo, I-80126 Napoli, Italy.

${ }^{\dagger}$ On leave from Institut de Physique Théorique, Université Paris Saclay, CNRS, CEA, F-91191 Gif-sur-Yvette, France.

${ }^{*}$ Corresponding author. orazio.scarlatella@gmail.com

${ }^{\S}$ Corresponding author. marco.schiro@college-de-france.fr

Published by the American Physical Society under the terms of the Creative Commons Attribution 4.0 International license. Further distribution of this work must maintain attribution to the author(s) and the published article's title, journal citation, and DOI.
}

\author{
Subject Areas: Condensed Matter Physics, \\ Quantum Physics, \\ Strongly Correlated Materials
}

\section{INTRODUCTION}

Developments in quantum science and quantum engineering have brought forth a variety of platforms to store, control, and process information at genuine quantum levels. Examples include trapped atoms and ions [1], quantum cavity-QED systems [2], superconducting qubits [3], or quantum optomechanical systems [4]. These architectures are of great relevance not only for quantum technologies but also for the quantum simulation of emergent collective many-body phenomena. We now have several experimental quantum simulators worldwide running on a variety of architectures, from ultracold atoms in optical lattices [5], Rydberg gases [6], trapped ions [7], and coupled cavity arrays [8]. Such simulators have led to significant advances 
in our understanding of quantum many-body phases and offer us an opportunity to address deep unanswered questions concerning the behavior of quantum matter in novel unexplored regimes, particularly far away from thermal equilibrium.

Many of these systems are typically characterized by excitations with a finite lifetime, due to unavoidable losses, dephasing, and decoherence processes originating from their coupling to external environments and, therefore, feature an intrinsic nonequilibrium nature. Arrays of circuit QED cavities, for example, are emerging as a unique platform for the quantum simulation of driven-dissipative quantum many-body systems [8-11], where the balance of drive and loss processes and the presence of strong nonlinearities allows one to reach interesting nonequilibrium stationary states. Experiments have recently started to show a variety of dissipative quantum phases and phase transitions [12-15], including the recent experimental realization of a dissipatively stabilized Mott insulator [16]. On a different front, recent advances with ultracold atoms in optical lattices allow the engineering of controlled dissipative processes, such as correlated losses [17,18] or heating due to spontaneous emission $[19,20]$, and to study the resulting quantum many-body dynamics over long timescales. These experimental settings offer fresh new inputs for quantum simulation of strongly correlated driven-dissipative quantum many-body systems, at the interface between quantum optics and condensed matter physics [21-26].

From a theoretical standpoint, these experimental platforms can be well described as open Markovian quantum systems, of either fermionic or bosonic particles or quantum spins, evolving through a Lindblad master equation for the many-body density matrix $\rho$ [27]:

$$
\partial_{t} \rho=-i[H, \rho]+\sum_{\alpha}\left(L_{\alpha} \rho L_{\alpha}^{\dagger}-\frac{1}{2}\left\{L_{\alpha}^{\dagger} L_{\alpha}, \rho\right\}\right) .
$$

The crucial aspect of this problem lies in the interplay between unitary dynamics and the dissipative evolution controlled by jump operators $L_{\alpha}$, out of which nontrivial stationary states can be engineered [28-31]. Open Markovian quantum many-body systems are predicted to display a broad range of new transient dynamical phenomena [32-35] and dissipative quantum phase transitions [36-41].

Solving the Lindblad equation for many-body systems is extremely challenging. Exact numerical solutions based on the diagonalization of the Lindbladian superoperator, or direct time evolution, are limited to very small systems, and only slightly larger systems can be addressed with quantum trajectories [42]. For one-dimensional systems, an efficient representation of the problem in the language of matrix product operator is possible $[43,44]$ and has been successfully used in the recent past [45]; however, its extension to higher-dimensional systems poses problems, although some recent results have been obtained [46-48]. As a result, a number of theoretical approaches have been developed in recent years to tackle driven-dissipative lattice systems [47,49-59]. Driven-dissipative correlated bosons, such as those described by Bose-Hubbard and related models, are particularly challenging to tackle numerically due to the unbounded Hilbert space.

A powerful nonperturbative approach to quantum lattice models, based on the limit of large lattice connectivity $z$ $[60,61]$, is provided by the dynamical mean-field theory (DMFT) of correlated electrons [62] and bosons [63-65]. When applied to equilibrium lattice bosons, DMFT captures, nonperturbatively, the $1 / z$ corrections to Gutzwiller mean-field theory through the solution of a quantum impurity model. Originally introduced for equilibrium problems, DMFT has been successfully applied to a variety of nonequilibrium fermionic problems with or without dissipation [66], including Markovian fermions [67], and to study unitary dynamics of correlated bosons [68].

In this work, we introduce a new approach to Markovian bosonic quantum many-body systems. Specifically, we extend the bosonic nonequilibrium DMFT [68] to systems evolving under the Lindblad master equation (1) and combine it with a strong coupling impurity solver tailored for correlated dissipative processes described by manybody jump operators. In the large connectivity limit, DMFT maps the Lindblad dynamics (1) onto a dissipative quantum impurity model describing a single interacting bosonic mode, subject to many-body Markovian dissipation, coupled to a coherent field and a non-Markovian environment, both to be determined self-consistently. The non-Markovian DMFT bath describes the effect of hopping processes from neighboring sites, which are completely missed by Gutzwiller mean-field theory. We show that these processes are particularly interesting in open quantum systems, since they not only introduce coherent effects but also provide new dissipative channels. Solving the resulting quantum impurity model in the presence of multiple manybody jump operators remains highly nontrivial, and stateof-the-art impurity solvers for nonequilibrium DMFT cannot be efficiently used in this setting. Here, we build upon our recent work on Markovian impurity models [69] to develop and benchmark a DMFT solver for drivendissipative bosonic systems. This approach is based on a superoperator hybridization expansion and resummation of an infinite class of diagrams known as noncrossing approximation (NCA).

As first application of the DMFT+NCA method, we study a Bose-Hubbard model subject to two-particle losses and single-particle incoherent pumping. This model is directly relevant for recent experiments with ultracold bosonic atoms in optical lattices under controlled dissipation $[18,20]$ as well as for arrays of superconducting circuits [16]. We predict the emergence of a dissipative phase transition from a normal to a superfluid phase, where above a critical hopping or pump strength the system 
spontaneously develops a coherent field oscillating in time, and discuss the effect of finite-connectivity corrections to Gutzwiller mean-field theory. We highlight how this transition can be naturally interpreted as the onset of many-body synchronization in an array of quantum Van der Pol oscillators, a phenomenon which recently attracted lots of attention [70-82]. We show that DMFT allows one to predict results which are qualitatively missed by Gutzwiller mean-field theory, including the redistribution of steady-state population and the suppression of gain due to hopping processes, a stationary quantum-Zeno regime, and a new competition between coherent and dissipative processes toward symmetry breaking. These results reflect in large quantitative corrections to the Gutzwiller meanfield phase diagram.

The paper is organized as follows. In the next section II, we summarize the main concepts and results of this work, which are presented in more detail in the rest of the paper. In Sec. III, we introduce DMFT in more detail and discuss its physical content. In Sec. IV, we discuss two methods to solve the quantum impurity problem: a strong coupling perturbative approach and a more powerful selfconsistent NCA method. In Sec. V, we present our results for an interacting Bose-Hubbard driven-dissipative lattice problem. Section VI is devoted to conclusions. In the Appendixes, we provide technical details on the derivation of DMFT (Appendix A), a nontrivial consistency check on the NCA (Appendix B), and various analytical results quoted in the main text (Appendixes $\mathrm{C}-\mathrm{G}$ ).

\section{SUMMARY OF MAIN RESULTS}

In this section, we present a summary of the main results of this work, which are discussed more in detail in the rest of the paper. In particular, in Sec. II A, we discuss the formulation of DMFT for Markovian bosons and of the NCA impurity solver, and, in Sec. II B, we discuss our theoretical approach in relation to prior work on open quantum many-body systems, while, in Sec. II C, we present the application to a driven-dissipative Bose-Hubbard model with two-body losses and a single-particle incoherent pump.

\section{A. Dynamical mean-field theory for open Markovian bosons}

The class of models that we consider describe drivendissipative bosonic particles on a lattice with coordination number $z$. The many-body density matrix of the system evolves according to a Lindblad master equation

$$
\partial_{t} \rho=-i[H, \rho]+\sum_{i \mu}\left(L_{i \mu} \rho L_{i \mu}^{\dagger}-\frac{1}{2}\left\{L_{i \mu}^{\dagger} L_{i \mu}, \rho\right\}\right)
$$

with a set of local jump operators for each lattice site $L_{i \mu}$, accounting for dissipative processes, and with coherent evolution governed by the Hamiltonian

$$
H=-\frac{J}{z} \sum_{\langle i j\rangle}\left(b_{i}^{\dagger} b_{j}+\text { H.c. }\right)+\sum_{i} H_{\mathrm{loc}}\left[b_{i}^{\dagger}, b_{i}\right] .
$$

Here, $\left[b_{i}, b_{i}^{\dagger}\right]=1$ are bosonic modes localized around the lattice site $i$, coupled together by a nearest-neighbor hopping term $J . H_{\mathrm{loc}}\left[b_{i}^{\dagger}, b_{i}\right]$ is a generic local Hamiltonian, which can contain arbitrary local interactions. In order for the problem to remain well defined in the limit of infinite connectivity $z \rightarrow \infty$, to which we compare our DMFT results, we scale the hopping with a $1 / z$ factor which is the correct scaling for bosons $[63,64,83]$ (see also Sec. III A for further details).

The DMFT approach considers the master equation (2) in the limit of large, but finite, lattice connectivity $z \gg 1$. In fact, when the number of neighbors $z$ of a given lattice site is large, statistical and quantum fluctuations induced by the neighboring sites get small and can be treated in an approximate way, while the local, on-site physics is accounted for exactly. As we discuss in detail in Sec. III, in the $z \gg 1$ limit, the Lindblad lattice problem formally maps onto a quantum impurity model describing an interacting Markovian single site, characterized by the same local Hamiltonian $H_{\text {loc }}$ and local jump operators $L_{i \mu}$ entering Eq. (1), coupled to a time-dependent field $\Phi_{\text {eff }}(t)$ acting as a coherent drive and a non-Markovian quantum bath (Fig. 1, top). These take into account the effect of the neighboring sites and have to be determined selfconsistently through the calculation of impurity properties. As a result of the nonequilibrium nature of the problem, the non-Markovian environment is described in terms of two independent bath hybridization functions, the retarded $\Delta^{R}\left(t, t^{\prime}\right)$ and Keldysh $\Delta^{K}\left(t, t^{\prime}\right)$, which in a stationary state encode information about the spectrum and occupation of the single-particle excitations. In a generic nonequilibrium condition, these are independent and not related by the fluctuation-dissipation theorem.

To appreciate the physical content of DMFT, it is instructive to compare it with the widely used Gutzwiller mean-field theory. As we show in Sec. III A, the latter coincides with the $z \rightarrow \infty$ solution of the many-body master equation and corresponds to DMFT when the non-Markovian bath is set to zero. Gutzwiller mean-field theory amounts to decoupling the hopping term in the Hamiltonian (3) or, equivalently, assumes a product-state density matrix over different lattice sites, thus reducing the full many-body problem to a single site coupled to a self-consistent coherent field (Fig. 1, bottom). An obvious shortcoming of the Gutzwiller approach is that it cannot capture any coherent or dissipative processes arising from the coupling to neighboring sites, unless the system is in a broken-symmetry phase with a nonvanishing local order parameter, leading to a finite self-consistent field. The result is a particularly poor description of strongly interacting, yet incoherent, normal phases such as bosonic Mott insulators or 




FIG. 1. Sketch of the DMFT mapping for open Markovian bosonic quantum systems. A lattice of interacting, driven-dissipative bosons coupled by hopping [left; see Eqs. (2) and (3) in the main text] is mapped in the large connectivity limit $z \gg 1$ onto a single-site problem, with interactions, local Markovian drive, and losses, coupled to a self-consistent environment (right, top). This single-site problem includes a coherent drive and a non-Markovian bath [characterized by two independent hybridization functions $\Delta^{R}\left(t, t^{\prime}\right)$ and $\left.\Delta^{K}\left(t, t^{\prime}\right)\right]$ which accounts for fluctuations due to neighboring sites. In the infinite coordination limit $z=\infty$, one recovers the Gutzwiller mean-field theory (right, bottom), where the effect of the lattice is simply encoded in a self-consistent field.

many-body quantum-Zeno phases that we discuss in this work, whose local properties within Gutzwiller are completely independent on the hopping and identical to those of an isolated site. In this perspective, DMFT accounts nonperturbatively, through the solution of a quantum impurity model with a non-Markovian bath $\Delta \sim 1 / z$, for finiteconnectivity corrections to Gutzwiller mean-field theory, thus capturing fluctuations induced by the neighboring sites even in the absence of an order parameter.

Although simplified with respect to the full master equation, the solution of DMFT equations and, in particular, of the quantum impurity problem sketched in Fig. 1 still poses tremendous challenges. In particular, the presence a Markovian environment containing arbitrary, possibly nonlinear, jump operators, in addition to local interactions and the non-Markovian DMFT bath, makes this problem hard to solve efficiently with state-of-the-art approaches for nonequilibrium DMFT. A major result of the present work is the development and benchmark of an impurity solver for Markovian bosonic DMFT based on the superoperator hybridization expansion formulated in Ref. [69] and applied there to a simple noninteracting fermionic resonant level model. As we discuss more in detail in Sec. IV B, this approach fully captures the underlying Markovian dynamics of the impurity problem in Fig. 1 and accounts for the non-Markovian bath $\Delta$ through the resummation of an infinite class of diagrams in the impurity-bath coupling known as NCA.

As we discuss further on in the paper, the self-consistent nature of the NCA we use, as opposed to bare perturbative expansions to which we compare our results, is crucial to fully capture the nontrivial correlations associated to the DMFT bath. We give a more complete picture of the DMFT formalism, including a discussion of the basic equations and of impurity solvers in Secs. III and IV.

\section{B. Relation to prior works}

Here, we wish to relate our approach with respect to previous works on nonequilibrium dissipative many-body systems. In the context of fermionic nonequilibrium DMFT, dissipation at the single-particle level (i.e., tunneling to external metallic leads) has been included before in several works, focusing, for example, on steady-state transport [84-89], Floquet-driven systems [90-92], or photodoping [93]. We note that this type of dissipation is straightforward to handle within DMFT and poses no additional methodological challenges, since it can be included within any impurity solver used for nonequilibrium DMFT in the absence of dissipation. On the other hand, many-body dissipative processes, such as those we focus on here in the Lindblad context or those modeling the coupling between fermions and bosonic baths, are more challenging to handle, since they induce effective interactions. Up to date, these have been included in nonequilibrium DMFT studies of dissipative problems only through perturbative expansions [94-98]. In this respect, our work goes beyond those studies in that all Markovian dissipative couplings (single and many-body) are treated on the same footing and encoded in the local Lindbladian of 
the impurity model, which opens up the possibilities for nonperturbative treatments of those couplings. This strategy is similar in spirit to what is done for Markovian fermionic systems in Ref. [67], where a discretization of the DMFT bath is used to solve the impurity problem with exact diagonalization. Here, instead, we use the NCA impurity solver, which works directly in the thermodynamic limit of an infinite bath and does not introduce any discretization, which would be particularly severe for bosonic problems such as the one we focus on here.

In the context of Markovian quantum many-body systems, there have been recent methodological developments to include correlations beyond mean-field theory. Although a precise comparison with DMFT is beyond the scope of this work, it is worth discussing some of those methods here. The cluster mean-field theory [51], developed for driven-dissipative quantum spin models, is similar to DMFT in that it adds short-range correlations on top of a Gutzwiller mean field, although this addition is achieved through the exact solution of a finite-size cluster rather than through an infinite (noninteracting) bath. The corner-space renormalization method [49] performs a diagonalization of the Lindbladian in a corner of the full Hilbert space, whose size is iteratively increased. As opposed to DMFT, which works in the thermodynamic limit, this method is finite size, which can, however, produce converged results for sizes much larger than brute-force methods [99]. Both those approaches focus naturally on static correlations encoded in the stationary-state density matrix, while DMFT is constructed around the frequency-resolved Green's functions.

\section{Application to a driven-dissipative Bose-Hubbard lattice}

In this work, we apply DMFT to a lattice model of driven-dissipative interacting bosons by specifying the local Hamiltonian and local jump operators entering Eqs. (2) and (3). We consider for the former

$$
H_{\mathrm{loc}}=\omega_{0} b_{i}^{\dagger} b_{i}+\frac{U}{2}\left(b_{i}^{\dagger} b_{i}\right)^{2},
$$

i.e., a characteristic frequency $\omega_{0}$ and on-site Kerr nonlinearity of strength $U$, while for the latter we consider two kinds $(\mu=1,2)$ of jump operators for each lattice site $i$ :

$$
\begin{aligned}
& L_{i 2}=\sqrt{\eta} b_{i} b_{i}, \\
& L_{i 1}=\sqrt{r} \sqrt{\eta} b_{i}^{\dagger} .
\end{aligned}
$$

We emphasize the correlated nature of the dissipative process encoded by $L_{i 2}$ which acts only on states with multiple bosonic occupancy. This term plays a key role for our results. Interestingly, this kind of loss process can be realized both with ultracold atoms $[17,18]$ as well with superconducting circuits [100]. The resulting lattice model [Eqs. (3)-(6)], therefore, describes a driven-dissipative realization of the Bose-Hubbard model [83], whose properties in the presence of incoherent drive and dissipation has been the subject of tremendous attention recently [32,40,54,101-109]. The specific form of dissipation we consider in Eq. (5) is rather unexplored in a many-body context, although few results are available in the literature that we recall briefly here.

The many-body master equation (1), (4)-(6) has a global $\mathrm{U}(1)$ symmetry, associated with the invariance of the Liouvillian with respect to the transformation $b_{i} \rightarrow b_{i} e^{i \theta}$, and is time translational invariant (TTI). In the limit of a large number of bosons per site, one expects a semiclassical description to work. The model reduces then to a discretized version of the Gross-Pitaevskii equation, largely studied in the context of exciton-polariton condensation [36], which predicts a coherent phase of bosons for any $r>0$, independently of $J / U$. This phase, which spontaneously breaks both the $\mathrm{U}(1)$ and TTI symmetry, corresponds to a nonequilibrium superfluid. In the opposite regime of uncoupled sites, $J / U=0$, the steady-state density matrix is known analytically from Refs. [110,111] and describes an incoherent state: It is a statistical mixture of Fock states with $\left\langle b_{i}\right\rangle=0$, as might be expected given the lack of any coherent driving. How those two different phases are connected upon increasing $J / U$, in the quantum regime of few bosons per site and finite lattice connectivity, is one of the main focuses of this paper.

In Fig. 2, we plot the DMFT phase diagram for our BoseHubbard model as a function of $r$ (the dimensionless pumpto-loss ratio) and $J / U$, for different values of the lattice connectivity $z$, together with the Gutzwiller mean-field phase boundary corresponding to the $z=\infty$ limit [112]. For a given fixed value of $z$, we find a critical line $r_{c}(J)$ separating a small-hopping normal phase where the bosons remain incoherent, $\left\langle b_{i}\right\rangle=0$, from a large-hopping phase where the system develops a local order parameter breaking the $\mathrm{U}(1)$ symmetry of the master equation. A first striking result that clearly appears from Fig. 2 is that, upon decreasing the connectivity, i.e., increasing the strength of fluctuations on top of the Gutzwiller mean-field result, the phase diagram changes substantially. In particular, the broken symmetry phase shrinks and moves toward larger values of pump and hopping. Interestingly, the DMFT corrections to the phase diagram turn out to be substantially larger than for equilibrium lattice bosons $[64,65,68]$. The effect of finite-connectivity fluctuations is, however, not only quantitative. As we discuss below, and more extensively in Sec. V, these corrections arise from a qualitatively new physics that is captured by the DMFT NCA description of the normal phase and completely missed by Gutzwiller mean-field theory.

As we discuss in more detail in Secs. VA and VB, the normal phase of our model comes with unique 




FIG. 2. Phase diagram of the Bose-Hubbard model [see Eqs. (3)-(5)] obtained with DMFT and NCA, as a function of pump/loss ratio $r$ and hopping/interaction ratio $J / U$, for different values of the lattice connectivity $z$, compared to the Gutzwiller mean-field one $(z=\infty)$. A critical line $r_{c}(J)$ separates a normal low-hopping phase from a broken symmetry phase where the system develops a local order parameter oscillating in time at finite frequency. Decreasing the lattice connectivity, i.e., increasing quantum fluctuations due to the finite number of neighbors, the ordered phase shrinks and the entire phase boundary is reshaped. In particular, the reentrant behavior of the normal phase found in the mean field disappears at small values of $z$. Parameters: $\eta / U=0.02, d t=0.004, t_{\max }=10$, and $\operatorname{dim}_{H}=10$ (see Sec. V).

nonequilibrium properties, inherited from the local manybody physics of the single-site problem [113]. Above a pump threshold $r_{\mathrm{NDOS}}$, the system develops a negative density of states (NDOS) at positive frequencies, a signature of incipient gain, i.e., energy emission in response to a weak coherent drive. Upon further increasing the pumpto-loss ratio above $r_{\text {inv }}>r_{\text {NDOS }}$, the steady-state reduced density matrix shows population inversion; namely, higherenergy states become more occupied than lower-energy ones. Within Gutzwiller mean-field theory, which describes the normal phase as a product state of single sites, those scales are independent from the hopping $J$. DMFT instead shows that fluctuations due to finite connectivity reshape completely the spectral and distribution properties of the normal phase, leading, in particular, to a suppression of NDOS and population inversion. This suppression arises from hopping-induced losses, a hallmark of the interplay between coherent and dissipative dynamics in our model, which are the key physics captured by DMFT through the non-Markovian bath.

In Sec. V C, we show that an interplay of NDOS and sufficiently strong hopping $J$ controls the true normal phase instability for values of the pump above $r_{c}(J)$, when the system develops full phase coherence and enters the superfluid phase. In particular, we find that the unstable mode is modulated in time and that the system displays a finite-frequency phase transition corresponding to an order parameter which develops undamped oscillations, thus breaking TTI $[109,114,115]$. The large reduction of the normal phase at finite connectivity can be interpreted as an effect due to hopping-induced losses arising from the non-Markovian DMFT bath, which is able to wipe out the NDOS and absorb part of the energy emitted by the system, as we discuss more in detail in Sec. V C 2. This mechanism for the destruction of an ordered phase is of genuine nonequilibrium origin and cannot be understood in terms of an effective heating. Indeed, as we show in Sec. V C 3, while an effective thermalization is captured by DMFT through an effective temperature, this result remains comparable to the Gutzwiller mean-field result up to small values of the connectivity and, therefore, cannot explain by itself the substantial reshape of the phase diagram.

The finite-frequency transition toward an oscillating nonequilibrium superfluid shares similarities with phenomena such as lasing, limit cycles, and synchronization. As we show more in detail in Sec. VD, the driven-dissipative Bose-Hubbard (3)-(5) reduces in the semiclassical limit to an array of coupled classical Van der Pol (VdP) oscillators [116-118], which admits a stable limit cycle phase, a coherent phase with an order parameter oscillating in time at finite frequency, for any finite pump $r>0$ and any coupling $J$. In the quantum regime of few bosons per site, the picture qualitatively changes, and a transition arises as a function of hopping $J$ depicted in Fig. 2. This transition can be interpreted, in light of this analogy, as a many-body quantum synchronization [70-82,119], where above a certain coupling $J$ all quantum $\mathrm{VdP}$ oscillators enter into a collective limit cycle phase.

Finally, in order to highlight the role of hopping-induced dissipative processes and the qualitative differences between DMFT NCA and Gutzwiller, in Sec. VE, we consider the limit of large two-body losses $\eta \gg J$ for our Bose-Hubbard model. We note that this regime is experimentally accessible with ultracold gases in the presence of inelastic collisions $[17,18]$. For large two-body losses, and in the absence of any external pump, perturbation theory shows that one can restrict the Bose-Hubbard dynamics to a subspace made by hard-core bosonic states, the dark states of the local dissipator [120]. Within this quantum-Zeno subspace $[121,122]$, the dominant dissipative processes left are those among neighboring sites, controlled by a hopping-induced loss rate $\Gamma_{\text {eff }}=\left(2(J / z)^{2} / U^{2}+\eta^{2}\right) \eta$. This scale is shown to affect the transient dynamics $[17,120,123]$, featuring a power-law decay toward the trivial zero-density steady state. Here, we use DMFT NCA to show that in the presence of an additional small residual pump $f \ll \eta$ one can stabilize a quantum-Zeno stationary state in which physical properties are controlled by the scale $\Gamma_{\text {eff }}$ (see Sec. V E for a detailed discussion). An example is provided in Fig. 3, where we plot the DMFT NCA steady-state occupation probability of the $n=1$ 


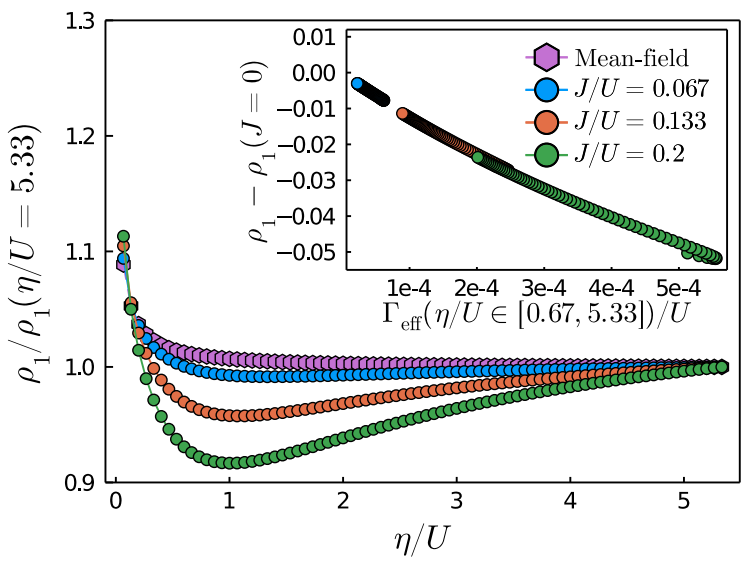

FIG. 3. Emergence of a quantum-Zeno regime at fixed drive rate $f / U=r \eta / U=0.013 z=6, U=15$ and increasing two-particleloss rate $\eta$. Parameters: $t_{\max }=20, d t=0.002$, and $\operatorname{dim}_{H}=6$. Probability of having one boson per site in the steady state $\rho_{1}$ as a function of $\eta / U$, rescaled by its value at $\eta / U=2.67$ for convenience. It follows a nonmonotonic behavior with a minimum at $\eta=U$, which is more pronounced the larger the hopping $J$. This behavior is a manifestation of quantum-Zeno physics, controlled by the hopping-induced loss rate $\Gamma_{\text {eff }}$ as we show clearly in the inset, which is instead completely missed by Gutzwiller.

bosonic state versus $\eta / U$, for different values of $J / U$, and compare it with Gutzwiller results. The latter shows, independently of $J$, a very weak dependence from $\eta$ which would be completely absent if not for the small residual pump. DMFT results instead show a clear nonmonotonic behavior as $J / U$ increases, with a minimum at $\eta \sim U$. This behavior is a signature of the emergence of the Zeno scale $\Gamma_{\text {eff }}$ controlling the physics, as we clearly reveal in the inset, where a scaling collapse is shown. We emphasize that the emergence of a quantumZeno stationary state represents a stringent test for the ability of DMFT of capturing hopping-induced losses, which are instead completely missed by Gutzwiller meanfield theory.

We give a more complete picture of our results for the Bose-Hubbard model in Sec. V.

\section{DYNAMICAL MEAN-FIELD THEORY FOR MARKOVIAN BOSONS}

In this section, we present the formalism of DMFT for Markovian bosons, including the basic self-consistency equations, its formal relation with Gutzwiller mean-field theory, and its physical interpretation, leaving its derivation to Appendix A. The starting point is to cast the Lindblad master equation (2) in the language of nonequilibrium Keldysh field theory, as discussed extensively in the literature [52]. The result is an action written in terms of bosonic coherent fields $\bar{b}_{i \alpha}$ and $b_{i \alpha}$ on each lattice site $i$ and on the upper and lower Keldysh contours, $\alpha= \pm$, which takes the form

$$
\mathcal{S}=\int_{-\infty}^{\infty} d t \sum_{i \alpha} \alpha \bar{b}_{i \alpha} i \partial_{t} b_{i \alpha}-\int_{-\infty}^{\infty} d t i \mathcal{L},
$$

where the Lindbladian $\mathcal{L}$ is given by

$$
\begin{aligned}
\mathcal{L}= & -i\left(H_{+}-H_{-}\right) \\
& +\sum_{\mu, i} \gamma_{\mu}\left(L_{i \mu+} \bar{L}_{i \mu-}-\frac{1}{2} \bar{L}_{i \mu+} L_{i \mu+}-\frac{1}{2} L_{i \mu-} \bar{L}_{i \mu-}\right)
\end{aligned}
$$

with $H_{\alpha}$ and $L_{i \mu \alpha}$ the expectation values of the Hamiltonian (3) and of the jump operators (5), respectively, expressed in terms of creation and annihilation operators belonging to the $\alpha$ contour, taken on bosonic coherent states. The full solution of the Keldysh action in Eq. (7) is, of course, not possible, in general, due to the coupling between many interacting modes and the presence of interaction, drive, and dissipation.

The key idea of DMFT is to write down an effective Keldysh action for the boson field of a single site of the lattice, obtained after integrating out all its neighbors [68]. As we show in Appendix A, in the limit of large lattice connectivity, $z \gg 1$, this effective action has the closed form

$$
\begin{aligned}
\mathcal{S}_{\mathrm{eff}}\left[\mathbf{b}_{\alpha}^{\dagger}, \mathbf{b}_{\alpha}\right]= & S_{\mathrm{loc}}\left[\mathbf{b}_{\alpha}^{\dagger}, \mathbf{b}_{\alpha}\right]+\int_{-\infty}^{\infty} d t \sum_{\alpha= \pm} \alpha \boldsymbol{\Phi}_{\mathrm{eff} \alpha}^{\dagger}(t) \mathbf{b}_{\alpha}(t) \\
& -\frac{1}{2} \int_{-\infty}^{\infty} d t d t^{\prime} \sum_{\alpha, \beta= \pm} \alpha \beta \mathbf{b}_{\alpha}^{\dagger}(t) \Delta^{\alpha \beta}\left(t, t^{\prime}\right) \mathbf{b}_{\beta}\left(t^{\prime}\right),
\end{aligned}
$$

where $\alpha$ and $\beta$ are Keldysh contour indices and we drop the site index from the local boson field for simplicity (we assume translational invariance) and group together creation and annihilation fields into a Nambu field

$$
\mathbf{b}_{\alpha}^{\dagger}=\left(\bar{b}_{\alpha} b_{\alpha}\right), \quad \mathbf{b}_{\alpha}=\left(\begin{array}{c}
b_{\alpha} \\
\bar{b}_{\alpha}
\end{array}\right) .
$$

The above local Keldysh action describes a driven-dissipative quantum impurity model [69]. The first term in Eq. (9), $S_{\mathrm{loc}}\left[\mathbf{b}_{\alpha}^{\dagger}, \mathbf{b}_{\alpha}\right]$, is the local, on-site contribution of the original lattice action (7) and (8) and, therefore, includes interactions, as well as Markovian incoherent drive and dissipation leading to off-diagonal terms in Keldysh space. The second and third terms describe the feedback of the rest of lattice onto the site through its neighbors, in terms of an effective coherent drive $\boldsymbol{\Phi}_{\text {eff } \alpha}^{\dagger}(t)$ and an effective non-Markovian bath with hybridization function $\Delta^{\alpha \beta}\left(t, t^{\prime}\right)$. Both these quantities have to be determined self-consistently; in particular, the effective coherent drive reads 


$$
\boldsymbol{\Phi}_{\mathrm{eff} \alpha}^{\dagger}=J \boldsymbol{\Phi}_{\alpha}^{\dagger}(t)+\int_{-\infty}^{\infty} d t^{\prime} \sum_{\beta= \pm} \beta \boldsymbol{\Phi}_{\beta}^{\dagger}\left(t^{\prime}\right) \Delta^{\beta \alpha}\left(t^{\prime}, t\right)
$$

and has two contributions, the first coming from the average of the bosonic field as in Gutzwiller mean-field theory:

$$
\boldsymbol{\Phi}_{\alpha}^{\dagger}=\left\langle\mathbf{b}_{\alpha}^{\dagger}\right\rangle_{S_{\text {eff }}}
$$

and the second one coming from the non-Markovian bath, a nontrivial finite $z$ correction accounting for the feedback of neighboring sites on the local effective field $[64,65,68]$. This latter term, whose origin is discussed more in detail in Appendix A, plays a key role within DMFT, in particular, for what concerns the corrections to the phase diagram as we discuss in Sec. V C.

The self-consistency relation for the Green's function depends on the specific choice of the lattice. In the following, we use the simplified relation for the Bethe lattice [68]:

$$
\boldsymbol{\Delta}^{\alpha \beta}\left(t, t^{\prime}\right)=\frac{J^{2}}{z} \mathbf{G}^{\alpha \beta}\left(t, t^{\prime}\right),
$$

which directly relates the hybridization function of the nonMarkovian bath to the impurity connected Green's function

$$
\mathbf{G}^{\alpha \beta}\left(t, t^{\prime}\right)=-i\left\langle\mathbf{b}_{\alpha}(t) \mathbf{b}_{\beta}^{\dagger}\left(t^{\prime}\right)\right\rangle_{S_{\mathrm{eff}}}+i \boldsymbol{\Phi}_{\alpha}(t) \boldsymbol{\Phi}_{\beta}^{\dagger}\left(t^{\prime}\right) .
$$

The DMFT solution of the original Markovian lattice problem, thus, requires one to solve the Keldysh action (9), computing, in particular, the impurity Green's function (14) and the average of the bosonic field (12), for given values of the non-Markovian bath $\Delta$ and effective field $\Phi$, and to iterate Eqs. (11)-(13) until self-consistency.

\section{A. Limit of infinite coordination number: Gutzwiller mean-field theory}

It is instructive at this point to take explicitly the limit of infinite coordination number $z \rightarrow \infty$. In this limit, the DMFT effective action (15) becomes completely local in time:

$\mathcal{S}_{\mathrm{eff}}\left[\mathbf{b}_{\alpha}^{\dagger}, \mathbf{b}_{\alpha}\right]^{z=\infty} \stackrel{\infty}{\rightarrow} S_{\mathrm{loc}}\left[\mathbf{b}_{\alpha}^{\dagger}, \mathbf{b}_{\alpha}\right]+\int_{-\infty}^{\infty} \sum_{\alpha} \alpha d t \boldsymbol{\Phi}_{\mathrm{eff} \alpha}^{\dagger}(t) \mathbf{b}_{\alpha}(t)$,

since the non-Markovian bath scales as $1 / z$ [see Eq. (13)] and as such can be unfolded back into a master equation for a single-site density matrix $\rho$, which satisfies

$$
\partial_{t} \rho(t)=i\left[\mathbf{b}^{\dagger} \mathbf{\Phi}(t), \rho\right]+\mathcal{L} \rho(t),
$$

where $\mathcal{L}$ is the local part of the Lindbladian and the feedback from the neighboring sites is carried by
$\boldsymbol{\Phi}(t)=\operatorname{tr}[\mathbf{b} \rho(t)]$. This equation corresponds to a factorized Gutzwiller-like ansatz for the lattice many-body density matrix

$$
\rho_{\text {latt }}(t)=\prod_{i} \rho_{i}(t)
$$

where $i$ is the site index and $\rho_{i}(t) \equiv \rho(t)$ because of translational invariance. In other words, we explicitly show that, as for equilibrium or closed systems $[64,68]$ also for driven-dissipative lattice systems, the infinite-connectivity limit of bosons coincides with Gutzwiller mean-field theory. We note that, when $\boldsymbol{\Phi}=0$, this mean field describes completely uncoupled sites, while DMFT $(z<\infty)$ captures the feedback from neighboring sites through the selfconsistent bath $\Delta$. In the following section, we are going to add some physical intuition on how the DMFT action (9) describes the effect of neighboring sites through a fictitious non-Markovian bath.

\section{B. DMFT effective action in the basis of classical and quantum fields}

We now give a physical interpretation to the various terms entering the DMFT effective action in Eq. (9), in particular, to the non-Markovian term. It is useful to introduce the so-called classical and quantum components of the bosonic field:

$$
\begin{aligned}
& \mathbf{b}_{\mathrm{cl} / q}(t)=\frac{\mathbf{b}_{+} \pm \mathbf{b}_{-}}{\sqrt{2}}, \\
& \mathbf{b}_{\mathrm{cl} / q}^{\dagger}(t)=\frac{\mathbf{b}_{+}^{\dagger} \pm \mathbf{b}_{-}^{\dagger}}{\sqrt{2}},
\end{aligned}
$$

in terms of which we can rewrite the Keldysh action as

$$
\begin{aligned}
\mathcal{S}_{\mathrm{eff}}= & S_{\mathrm{loc}}\left[\mathbf{b}_{\mathrm{cl} / q}^{\dagger}, \mathbf{b}_{\mathrm{cl} / q}\right]+\int_{-\infty}^{\infty} d t \boldsymbol{\Phi}_{\mathrm{effcl}}^{\dagger} \mathbf{b}_{q} \\
& -\frac{1}{2} \int_{-\infty}^{\infty} d t d t^{\prime}\left[\mathbf{b}_{q}^{\dagger}(t) \Delta^{R}\left(t, t^{\prime}\right) \mathbf{b}_{\mathrm{cl}}\left(t^{\prime}\right)+\text { H.c. }\right] \\
& -\frac{1}{2} \int_{-\infty}^{\infty} d t d t^{\prime} \mathbf{b}_{q}^{\dagger}(t) \Delta^{K}\left(t, t^{\prime}\right) \mathbf{b}_{q}^{\dagger}\left(t^{\prime}\right) .
\end{aligned}
$$

In this basis, only two independent combinations of the non-Markovian kernels $\Delta^{\alpha \beta}$ enter, namely, the retarded component $\quad \boldsymbol{\Delta}^{R}\left(t, t^{\prime}\right)=\theta\left(t-t^{\prime}\right)\left[\boldsymbol{\Delta}^{-+}\left(t, t^{\prime}\right)-\boldsymbol{\Delta}^{+-}\left(t, t^{\prime}\right)\right]$, which couples the classical and quantum components of the field and encodes the spectral properties of the bath resulting in a frequency-dependent damping for the bosonic mode and the Keldysh component $\boldsymbol{\Delta}^{K}=$ $\boldsymbol{\Delta}^{-+}\left(t, t^{\prime}\right)+\boldsymbol{\Delta}^{+-}\left(t, t^{\prime}\right)$, encoding the occupation of the 
bath and resulting in a frequency-dependent noise for the bosonic mode. It is worth stressing that the above Keldysh action contains quadratic, non-Markovian terms in the classical and quantum fields as well as nonlinearities and higher powers of the classical and quantum fields included in the local part of the action $S_{\mathrm{loc}}\left[\mathbf{b}_{\mathrm{cl} / q}^{\dagger}, \mathbf{b}_{\mathrm{cl} / q}\right]$. While the structure of Eq. (18) is a generic feature of DMFT, the local part of the action depends on the particular form of local interaction and jump operators.

Finally, we can express also the impurity Green's functions Eq. (14) in this basis to obtain the retarded Green's function and the Keldysh one:

$$
\begin{aligned}
\mathbf{G}^{R}\left(t, t^{\prime}\right) & =-i\left\langle\mathbf{b}_{\mathrm{cl}}(t) \mathbf{b}_{q}^{\dagger}\left(t^{\prime}\right)\right\rangle_{S_{\mathrm{eff}}}+i \boldsymbol{\Phi}_{\mathrm{cl}}(t) \boldsymbol{\Phi}_{q}^{\dagger}\left(t^{\prime}\right), \\
\mathbf{G}^{K}\left(t, t^{\prime}\right) & =-i\left\langle\mathbf{b}_{\mathrm{cl}}(t) \mathbf{b}_{\mathrm{cl}}^{\dagger}\left(t^{\prime}\right)\right\rangle_{S_{\mathrm{eff}}}+i \boldsymbol{\Phi}_{\mathrm{cl}}(t) \boldsymbol{\Phi}_{\mathrm{cl}}^{\dagger}\left(t^{\prime}\right) .
\end{aligned}
$$

Those correlation functions contain crucial physical information about the local physics of the driven-dissipative lattice problem. The retarded Green's function, in particular, encodes information about the local excitation spectrum of the system, and it is known to be a crucial probe for the transition from delocalization to Mott-ness in strongly correlated systems at equilibrium [62]. For open Markovian quantum systems, the retarded Green's function contains, much like for closed equilibrium systems, information on the structure of the excitations on top of the stationary state [113], and it directly probes dissipative phase transitions where those excitations become unstable. Its poles correspond to eigenvalues of the Lindbladian in the singleparticle sector, which come with a characteristic frequency and lifetime, and their (possibly complex) weight. The retarded Green's function has also a directly physical meaning: It describes the linear response of the expectation $\langle b(t)\rangle$ to a weak, classical field $h\left(t^{\prime}\right)$, which couples linearly to $b^{\dagger}$. In the case where $b$ describes a photonic cavity mode, $G^{R}(t)$ can be directly measured by weakly coupling the cavity to an input-output waveguide and measuring the reflection of a weak probe tone (see, e.g., Refs. [124,125]).

The Keldysh Green's function, on the other hand, contains information about how the finite-frequency excitations above the stationary state are populated. In thermal equilibrium, those two functions are not independent but constrained to satisfy the fluctuation-dissipation theorem [126].

\section{Computing lattice quantities}

Solving the DMFT effective action and computing the impurity Green's functions (19) gives direct information on the local properties of the driven-dissipative lattice problem. Furthermore, one can access nonlocal quantities, such as momentum distribution or nonlocal correlation functions, through the lattice Green's functions at momentum $\mathbf{k}$ :
$\mathbf{G}_{\mathbf{k}}^{\alpha \beta}\left(t, t^{\prime}\right)=-i\left\langle\mathbf{b}_{\mathbf{k} \alpha}(t) \mathbf{b}_{\mathbf{k} \beta}^{\dagger}\left(t^{\prime}\right)\right\rangle+i \boldsymbol{\Phi}_{\mathbf{k} \alpha}(t) \boldsymbol{\Phi}_{\mathbf{k} \beta}^{\dagger}\left(t^{\prime}\right)$.

These satisfy a Dyson equation with a lattice self-energy $\boldsymbol{\Sigma}^{\alpha \beta}\left(t, t^{\prime}\right)$, that within DMFT is momentum independent [61,66]:

$\mathbf{G}_{\mathbf{k}}^{\alpha \beta}\left(t, t^{\prime}\right)=\mathbf{g}_{\mathbf{k}}^{\alpha \beta}\left(t, t^{\prime}\right)+\sum_{\gamma \delta} \mathbf{g}_{\mathbf{k}}^{\alpha \gamma} \otimes \mathbf{\Sigma}^{\gamma \delta} \otimes \mathbf{G}_{\mathbf{k}}^{\delta \beta}\left(t, t^{\prime}\right)$,

and coincides with the self-energy of the impurity problem

$\mathbf{G}^{\alpha \beta}\left(t, t^{\prime}\right)=\mathbf{g}^{\alpha \beta}\left(t, t^{\prime}\right)+\sum_{\gamma \delta} \mathbf{g}^{\alpha \gamma} \otimes \boldsymbol{\Sigma}^{\gamma \delta} \otimes \mathbf{G}^{\delta \beta}\left(t, t^{\prime}\right)$,

where in the above equations $\otimes$ indicates time convolutions, $\mathbf{g}^{\alpha \beta}\left(t, t^{\prime}\right)$ are the Green's functions of the quantum impurity problem with no interactions, but including the non-Markovian bath $\boldsymbol{\Delta}$, and $\mathbf{g}_{\mathbf{k}}^{\alpha \beta}\left(t, t^{\prime}\right)$ are the noninteracting lattice Green's functions.

\section{QUANTUM IMPURITY SOLVERS}

The main challenge behind our DMFT approach is to solve the Markovian quantum impurity model described by the Keldysh action (9), computing, in particular, the Green's functions. We stress that this task remains difficult due to the presence of interactions on the impurity site, nonlinear jump operators (such as our two-body losses), and the non-Markovian DMFT bath. While several impurity solvers have been developed in recent years for nonequilibrium DMFT [66], none of them can be efficiently applied in our case (see Sec. II B for a detailed discussion). To make progress, we take explicit advantage of the Markovian structure of the impurity, which allows us to treat nonlinear jump operators as dissipative couplings of a local Lindbladian. This treatment unleashes the possibility of developing strong-coupling impurity solvers for bosonic Markovian problems, which treat exactly the local Lindblad problem and include the effect of the nonMarkovian DMFT bath through perturbative or nonperturbative schemes. We note that for nonequilibrium closed systems these strong-coupling methods represent the current state of the art of DMFT impurity solvers [66]. Here, we develop two such schemes for bosonic Markovian systems, the Hubbard-I approximation and the more powerful noncrossing approximation, that we present below. We comment in Sec. VI on possible methodological extensions.

\section{A. Hubbard-I approximation}

The simplest approximation to solve the impurity problem (9) is based on perturbation theory in the nonMarkovian bath kernel $\boldsymbol{\Delta}$, and its lowest order is known as the Hubbard-I approximation [62,127]. As we see, this approach already gives a hopping dependence of 
correlation functions which goes beyond Gutzwiller meanfield theory but misses important correlations due to the non-Markovian bath. Our DMFT approach is based on the more powerful noncrossing approximation solver which we introduce in the next section, but we use Hubbard-I results for comparison and to motivate the need of a more powerful solver.

For simplicity, we formulate Hubbard-I in the normal phase, where $\boldsymbol{\Phi}=0$ and anomalous correlation functions vanish; thus, we can restrict to the first Nambu component and refer to it with nonbold symbols, e.g., $G^{\alpha \beta}=\mathbf{G}_{11}^{\alpha \beta}$, where $\alpha, \beta= \pm$ are Keldysh indexes. We also focus on the stationary-state regime, where Green's functions depend on time differences and we can move to the frequency domain, i.e., $G^{\alpha \beta}(\omega)$, which is the case we consider in our application in Sec. V.

The impurity Green's function obeys a Dyson equation [see Eq. (22)] in terms of a self-energy $\Sigma^{\alpha \beta}(\omega)$ which contains the effect of interaction, incoherent drive, and dissipation and which is, in general, a functional of the non-Markovian bath kernel $\Delta^{\alpha \beta}(\omega)$. Hubbard-I consists in approximating the impurity self-energy by its value for $\Delta=0$, i.e., in the absence of the bath, when it can be written as

$$
\Sigma^{\alpha \beta}(\omega) \approx\left[g_{0}^{\alpha \beta}(\omega)\right]^{-1}-\left[G_{0}^{\alpha \beta}(\omega)\right]^{-1} .
$$

Here, $G_{0}^{\alpha \beta}(\omega)$ is the Green's function of the impurity site with interaction, incoherent drive, and dissipation but without the bath (the latter condition is indicated by the index 0 ); it can be computed numerically [113]. In contrast, $g_{0}^{\alpha \beta}(\omega)$ corresponds to the Green's function of the impurity site in the absence of the bath and without interactions (lowercase letter), which is known analytically. Plugging this selfenergy back in the Dyson equation (22) and using the self-consistency condition on the Bethe lattice, we obtain a closed matrix equation for the Keldysh components of the local lattice Green's functions:

$$
\left[G^{\alpha \beta}(\omega)\right]^{-1}=\left[G_{0}^{\alpha \beta}(\omega)\right]^{-1}-\frac{J^{2}}{z} G^{\alpha \beta}(\omega) .
$$

The expressions of the retarded and Keldysh components are given explicitly in Appendix E. In the Appendix, we also show that the Hubbard-I approximation, despite introducing a beyond-mean-field hopping dependence of Green's functions, still yields the same phase diagram as mean-field theory, motivating the need for a more powerful solver.

\section{B. Superoperator hybridization expansion and noncrossing approximation}

To go beyond the Hubbard-I approximation, we build upon the method recently formulated in Ref. [69] and applied so far only to a simple toy model fermionic system, to develop a DMFT NCA impurity solver for bosonic
Markovian systems. The idea is to perform a diagrammatic expansion in powers of the non-Markovian bath $\boldsymbol{\Delta}$ and to resum an infinite set of diagrams by solving a selfconsistent Dyson-type equation. We remark that this expansion is carried out around an interacting problem, the single-site Markovian impurity; hence, it is not based on Wick's theorem as in weak-coupling perturbation theories. As such, working directly with Green's functions is not convenient and the more natural formulation is in terms of evolution superoperators, that we denote in the following with a hat [69]. We start by defining the evolution superoperator $\hat{\mathcal{V}}$ of the reduced density matrix of the impurity:

$$
\rho_{\text {imp }}(t)=\hat{\mathcal{V}}(t, 0) \rho_{\text {imp }}(0)
$$

formally obtained by tracing out the bath degrees of freedom. We note that Eq. (25) assumes that at time $t=0$ the non-Markovian bath is not entangled with the impurity site; i.e., in the original lattice problem, the initial condition corresponds to the limit of decoupled sites. Since the bath degrees of freedom are treated as noninteracting, only the single-particle Green's function of the bath enters the reduced dynamics, the hybridization function $\Delta$ introduced in Eq. (9). Expanding the superoperator $\hat{\mathcal{V}}(t, 0)$ in powers of $\boldsymbol{\Delta}$, we obtain a series which can be represented diagrammatically as shown in Fig. 4, where bold solid lines describe the propagator $\hat{\mathcal{V}}(t, 0)$ and dashed lines represent the hybridization function $\boldsymbol{\Delta}$, while simple solid lines represent the bare Markovian evolution superoperator $\hat{\mathcal{V}}_{0}(t, 0)=T \exp \left[\int_{0}^{t} d t^{\prime} \hat{\mathcal{L}}_{\text {eff }}\left(t^{\prime}\right)\right]$, where $T$ is the time ordering and $\hat{\mathcal{L}}_{\text {eff }}(t)=\hat{\mathcal{L}}_{0}+i\left[\hat{\mathbf{b}}^{\dagger} \hat{\boldsymbol{\Phi}}(t), \bullet\right]$ the effective single-site Lindblad superoperator with argument $\bullet$.

This diagrammatic representation allows one to define the self-energy $\hat{S}$ of the series as the sum of one-particleirreducible (1PI) diagrams, which cannot be cut into two disconnected parts by removing a solid line, and, thus, to formally resum the series into the Dyson equation

$$
\hat{\mathcal{V}}\left(t, t^{\prime}\right)=\hat{\mathcal{V}}_{0}\left(t, t^{\prime}\right)+\int_{t^{\prime}}^{t} d t_{1} \int_{t^{\prime}}^{t_{1}} d t_{2} \hat{\mathcal{V}}_{0}\left(t, t_{1}\right) \hat{S}\left(t_{1}, t_{2}\right) \hat{\mathcal{V}}\left(t_{2}, t^{\prime}\right)
$$

(a)

(b)

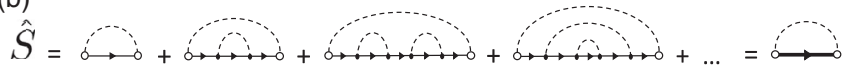

FIG. 4. (a) Diagrammatic representation of the Dyson series in Eq. (26). The bold (thin) solid lines represent the full, nonMarkovian (bare, Markovian) impurity superoperator $\hat{\mathcal{V}}\left(\hat{\mathcal{V}}_{0}\right)$, while the dashed lines correspond to the non-Markovian bath $\boldsymbol{\Delta}$. (b) Expression of the self-energy $\hat{S}$ in the NCA, where only diagrams with noncrossing $\Delta$ lines are retained whose sum can be explicitly evaluated; see Eq. (27). 
We remark that $\hat{\mathcal{V}}_{0}, \hat{\mathcal{V}}$, and $\hat{S}$ here are superoperators and that the self-energy $\hat{S}$ is a functional of the propagator $\hat{\mathcal{V}}$ whose closed form is not known, in general. The resulting series (26) generalizes to the case of Markovian impurities the hybridization expansion obtained for unitary quantum impurity models [128-132]. For the latter, exact resummation techniques based on diagrammatic Monte Carlo methods [133] are employed but generically suffer from the socalled sign problem, especially out of equilibrium, limiting the propagation time. Here, instead, we adopt a selfconsistent approximation for the self-energy $\hat{S}$. This approximation can be written, in general, as a systematic expansion in diagrams with an increasing number of crossing hybridization lines, an approach which is extensively used for unitary quantum impurity models [134-137]. The lowest-order contribution is given by noncrossing diagrams, e.g., in Fig. 4, giving an explicit expression for the NCA self-energy:

$$
\begin{aligned}
\hat{S}\left(t_{1}, t_{2}\right)= & -\frac{i}{2} \sum_{\substack{\alpha \beta \\
a b}} \alpha \beta\left[\Delta_{b a}^{\beta \alpha}\left(t_{1}, t_{2}\right) \hat{b}_{\beta b}^{\dagger} \hat{\mathcal{V}}\left(t_{1}, t_{2}\right) \hat{b}_{\alpha a}\right. \\
& \left.+\Delta_{a b}^{\alpha \beta}\left(t_{2}, t_{1}\right) \hat{b}_{\beta b} \hat{\mathcal{V}}\left(t_{1}, t_{2}\right) \hat{b}_{\alpha a}^{\dagger}\right] .
\end{aligned}
$$

In the above expression, $\alpha, \beta= \pm$ are Keldysh indices and $a, b=\{1,2\}$ are Nambu indices. Thus, $\Delta_{a b}^{\alpha \beta}$ is a given component of the bath hybridization function introduced in Eq. (9), i.e., $\Delta_{a b}^{\alpha \beta}=\left(\Delta^{\alpha \beta}\right)_{a b}$. We also introduce the superoperator analogs of the Nambu fields of Eq. (10), that we define as

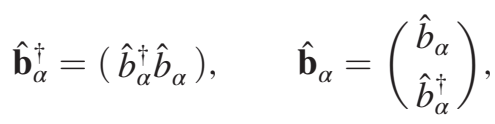

and denote their $a$ Nambu component as $\hat{b}_{\alpha a}$ in Eq. (27). The Keldysh index $\alpha= \pm$ for a superoperator specifies whether it should act from the left or the right of its argument, i.e.,

$$
\hat{b}_{+}=b \bullet, \quad \hat{b}_{-}=\bullet b,
$$

and similarly for $\hat{b}_{\alpha}^{\dagger}$. We notice that the self-energy depends on the full propagator $\hat{\mathcal{V}}$ rather than on the bare one $\hat{\mathcal{V}}_{0}$, thus containing diagrams to all orders in $\boldsymbol{\Delta}$. Corrections to the NCA can be obtained systematically including self-energy diagrams with higher numbers of crossings, although the resulting computational cost increases. In this work, where we focus on the normal phase and its instability, we limit ourselves to the NCA scheme, while we expect that, to access the superfluid phase or for lower values of the connectivity, higher-order corrections would become important. We note, in fact, that self-energy corrections including higher numbers of crossing diagrams come with higher powers of the DMFT bath, which for bosons is of the order of $J^{2} / z$ [see Eq. (13)] and, therefore, subleading at least for large to moderate values of the connectivity.

Once the self-energy $\hat{S}$ is known in closed form, the propagator $\hat{\mathcal{V}}$ can be obtained numerically by solving Eqs. (26) and (27). To use this NCA impurity solver in our DMFT approach, we need to compute the one-particle Green's functions of the impurity [Eq. (14)]. This value can be obtained by taking the functional derivative with respect to $\Delta$ of the partition function $Z=\operatorname{tr}\left[\rho_{\text {imp }}(\infty)\right]$ in Eq. (25) and using the Dyson equation for $\hat{\mathcal{V}}$ (see Appendix G). The final result reads

$$
\begin{aligned}
G_{a b}^{\alpha \beta}\left(t, t^{\prime}\right)= & -i\left\{\operatorname{tr}\left[\hat{b}_{\alpha a} \hat{\mathcal{V}}\left(t, t^{\prime}\right) \hat{b}_{\beta b}^{\dagger} \rho_{\mathrm{imp}}\left(t^{\prime}\right)\right] \theta\left(t-t^{\prime}\right)\right. \\
& \left.+\operatorname{tr}\left[\hat{b}_{\beta b}^{\dagger} \hat{\mathcal{V}}\left(t^{\prime}, t\right) \hat{b}_{\alpha a} \rho_{\mathrm{imp}}(t)\right] \theta\left(t^{\prime}-t\right)\right\} \\
& +i \Phi_{\alpha a}(t) \Phi_{\beta b}^{\dagger}\left(t^{\prime}\right),
\end{aligned}
$$

where as before we write explicitly both the Keldysh indices $\alpha$ and $\beta$ and the Nambu ones $a$ and $b$ and where $\Phi_{\alpha a}(t)=$ $\operatorname{tr}\left[\hat{b}_{\alpha a} \rho_{\text {imp }}(t)\right]$. We notice that this result, which resembles a quantum regression theorem [27] for the non-Markovian map $\hat{\mathcal{V}}\left(t, t^{\prime}\right)$, is valid only within NCA, while including higherorder diagrams into the self-energy leads to further terms which can be interpreted as vertex corrections.

Finally, we conclude by emphasizing that the solver introduced in this section is different from other NCA approaches to quantum impurity models with or without dissipation $[68,96,98,136,138]$, which treat at the noncrossing level all couplings to the baths. Here, by formulating the hybridization expansion at the superoperator level, we are able to fully capture the underlying local Markovian dynamics, resorting to an NCA only for the non-Markovian DMFT bath. This approach introduces several differences with respect to the NCA literature, including the way the Green's functions are evaluated [see Eq. (30)] and in the way the stationary-state theory is constructed, as we discuss further in the next section.

\section{Stationary-state DMFT/NCA}

While the formalism introduced so far allows us to compute the whole transient dynamics, in this section, we show how to directly address the stationary-state properties of the system within our DMFT NCA approach. At stationarity, we expect the local Green's functions (14) and, through the self-consistent condition (13), the bath hybridization function $\Delta^{\alpha \beta}$ to depend only on time differences. We can then solve the NCA Dyson equation (26) for the stationary-state propagator $\hat{\mathcal{V}}\left(t-t^{\prime}\right)$, which also depends only on time differences. This approach allows us to significantly reduce the computational cost for time propagating this equation from $O\left(t_{\max }^{3}\right)$ to $O\left(t_{\max }^{2}\right)$, where $t_{\max }$ is the maximum integration time. 
A complete steady-state DMFT NCA procedure requires one to compute, in addition to the stationary-state propagator, also the steady-state density matrix of the impurity $\rho_{s} \equiv \hat{\mathcal{V}}(\infty, 0) \rho_{\text {imp }}(0)$, which is needed to evaluate the impurity Green's functions [see Eq. (30)]. While, in principle, this constraint would require one to perform the full transient dynamics from an arbitrary initial condition, here we show how to obtain $\rho_{s}$ directly from the stationary-state propagator $\hat{\mathcal{V}}\left(t-t^{\prime}\right)$. We note that for Markovian open quantum systems the stationary-state density matrix can be directly obtained as zero eigenvalue of the Lindblad superoperator generating the dynamics. This argument, however, does not directly apply to the present case, since the DMFT bath makes the map $\hat{\mathcal{V}}\left(t, t^{\prime}\right)$ non-Markovian. A generalized stationarity condition for the non-Markovian map (26) can be obtained [69] by requiring the derivative of Eq. (26) to vanish at long times, i.e., $\lim _{t \rightarrow \infty} \partial_{t} \hat{\mathcal{V}}(t, 0) \rho_{0}=0$. This equation, however, still requires the knowledge of the full transient propagator. A major simplification arises in DMFT if the system reaches a stationary state, becoming time-translational invariant. Then, the condition for the impurity density matrix simplifies to (see Appendix F)

$$
\left(\hat{\mathcal{L}}_{\text {eff }}(\infty)+\int_{0}^{\infty} d \tau \hat{S}(\tau)\right) \rho_{s}=0
$$

where the self-energy $\hat{S}(\tau)$ depends only on the steady-state propagator $\hat{\mathcal{V}}(\tau)$ and not on the transient dynamics, allowing us to compute $\rho_{s}$ in a steady-state DMFT procedure. Equation (31) is analogous to the well-known condition for the stationary state of Markovian master equations, with an additional contribution of the nonMarkovian bath given by the time integral of the NCA self-energy. In practice, to solve DMFT NCA for the stationary state, we solve the Dyson equation (26) for $\hat{\mathcal{V}}(t)$ starting from an initial ansatz for $\boldsymbol{\Delta}\left(t-t^{\prime}\right), \boldsymbol{\Phi}\left(t^{\prime}\right)$, and $\rho_{s}$. As an initial condition, we usually compute these quantities from the steady-state solution of the single-site problem. Then, we compute the updated stationary density matrix $\rho_{s}$ using Eq. (31) and the updated $\boldsymbol{\Delta}\left(t-t^{\prime}\right), \boldsymbol{\Phi}\left(t^{\prime}\right)$ from Eqs. (11) and (13) and iterate until convergence is reached. We conclude by noting that, in principle, the stationary-state approximation could break down, leading to oscillatory behaviors. It is, therefore, important to study the stability of the steady state, which is encoded in the retarded Green's function as we discuss more in detail in Sec. V C.

\section{DMFT RESULTS FOR A DRIVEN-DISSIPATIVE BOSE-HUBBARD LATTICE}

In this section, we discuss our results for the drivendissipative Bose-Hubbard model introduced in Sec. II C, comparing different impurity solvers (NCA and Hubbard-I approximation) and highlighting the effect of introducing fluctuations beyond Gutzwiller mean-field theory due to the finite lattice connectivity. We start by discussing the properties of the normal phase at low hopping as encoded in its local spectral function (Sec. VA). We then move on to occupation properties of the nonequilibrium normal phase (Sec. V B) from the point of view of the local density and populations of the stationary-state reduced density matrix. In Sec. VC, we discuss the finite-frequency instability of the normal phase, leading to the DMFT NCA phase diagram, and provide a physical interpretation based on hoppinginduced dissipation for the large reduction of the ordered phase found in DMFT with respect to the Gutzwiller meanfield result. In Sec. V D, we connect the phase transition in our driven-dissipative Bose-Hubbard model to the physics of an array of quantum Van der Pol oscillators, in particular, to the onset of many-body synchronization and limit cycles, and discuss their fate at finite lattice connectivity. Finally, in Sec. VE, we discuss the regime of large two-body losses, where quantum-Zeno physics emerges and the qualitative differences between Gutzwiller and DMFT NCA results appear even more clearly.

Unless stated otherwise, we work in the regime where the interaction strength dominates the dissipation scale, i.e., we fix $\eta / U=0.02$, and study the model as a functions of the pump/loss ratio $r$ and the hopping-to-interaction ratio $J / U$. We set $U=5$ and $\omega_{0}=1$, although we note that this latter scale only sets the zero of energy and can be eliminated by going to a rotating frame, so it does not play any role in the physics.

We introduce a cutoff on the local Hilbert space $\operatorname{dim}_{H}$, whose value is specified for each result. We solve DMFT for the normal phase, where $\boldsymbol{\Phi}=0$ and the anomalous (Nambu) Green's function components vanish so that the self-consistent bath retains only Keldysh indexes $\Delta^{\alpha \beta}$. The NCA propagator in the stationary regime $\mathcal{V}(t)$ is obtained, as described in Sec. IV B 1, by propagating in time the derivative of the Dyson equation (26) assuming timetranslational invariance:

$$
\partial_{t} \hat{\mathcal{V}}(t)=\hat{\mathcal{L}}_{0} \hat{\mathcal{V}}(t)+\int_{0}^{t} d t_{1} \hat{S}\left(t-t_{1}\right) \hat{\mathcal{V}}\left(t_{1}\right)
$$

with an implicit second-order Runge-Kutta scheme [66], a propagation time $t_{\max }=10$, and a time step $d t=0.004$. We note that, in the regime under consideration in this work, the dynamics of the Dyson equation is dominated by the non-Markovian bath rather than by the two-particle losses, and, therefore, a $t_{\max }=10=1 / \eta$ is sufficient to reach convergence. Convergence of the implicit Runge-Kutta at each time step is assumed to be reached when $1 /\left(\operatorname{dim}_{H}\right)^{4} \sum_{j k}\left|\mathcal{V}_{j k}^{(i)}(t)-\mathcal{V}_{j k}^{(i-1)}(t)\right|<10^{-5}$, with $i$ the iteration index. The convergence of the DMFT scheme is assessed by checking that $1 /\left(2 t_{\max }\right) \sum_{\alpha} \int_{0}^{t_{\max }}\left|\left(\Delta^{\alpha,-\alpha}\right)^{(i)}(t)-\left(\Delta^{\alpha,-\alpha}\right)^{(i-1)}(t)\right|<10^{-5}$, 
with $i$ the index of the DMFT iteration. We check that our results essentially do not change by decreasing those thresholds or increasing the Hilbert space cutoff.

\section{A. Spectral function in the normal phase}

To characterize the properties of the system, we first focus on the local retarded Green's function defined in Eq. (19). Since in the normal phase all anomalous (Nambu) Green's function components vanish as well as the average of the order parameter, we have only one independent Nambu component $G^{R}(t)=-i \theta(t)\left\langle\left[b(t), b^{\dagger}(0)\right]\right\rangle$. Its imaginary part defines the local spectral function

$$
A(\omega)=-\frac{1}{\pi} \operatorname{Im} G^{R}(\omega)
$$

In Fig. 5, we plot the local spectral function in the low pump regime, $r=0.6$, for different values of $J / U$ and compare the DMFT NCA results with those obtained with Hubbard-I impurity solver and Gutzwiller mean-field theory.

The Gutzwiller mean-field spectral function shows a series of narrow peaks, whose broadening is controlled only by the local dissipation. We remark that in this approach, corresponding to the infinite coordination number limit $z=\infty$, all properties of the normal phase are independent on the hopping and coincide with the singlesite $J=0$ limit. Indeed, as we discuss in Sec. III A, for $z=\infty$ the only feedback from neighboring sites comes

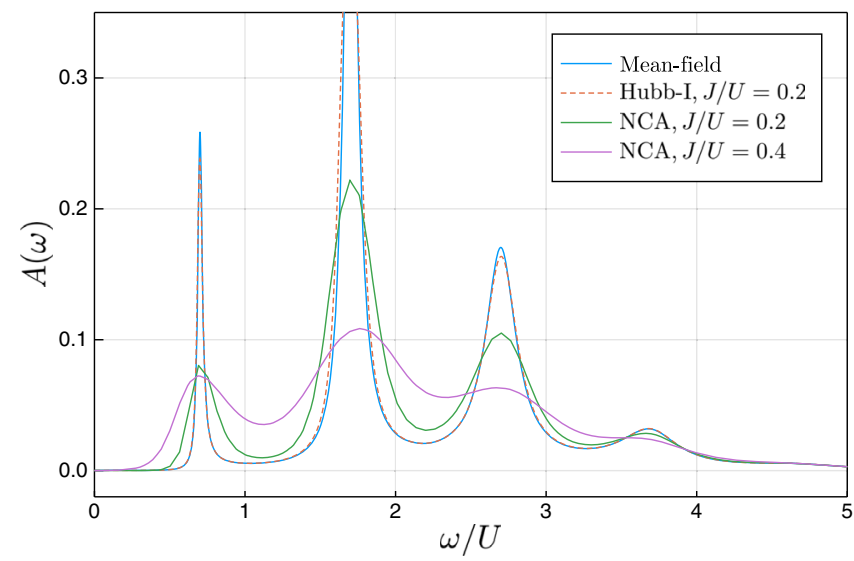

FIG. 5. Local spectral function $A(\omega)$ for different values of $J / U$, as computed within Gutzwiller mean-field theory (blue line), DMFT with Hubbard-I impurity solver (dashed red line) and NCA impurity solver (green and violet full lines), for fixed $z=6$ and $r=0.6$. Gutzwiller results show a series of narrow peaks broadened by the local dissipation only. DMFT instead is able to capture additional broadening processes, already evident for $J / U=0.2$ within Hubbard-I, which, however, largely underestimates the effect of the non-Markovian bath as confirmed by the comparison with the more accurate NCA. Parameters: $\operatorname{dim}_{H}=10$. through the order parameter $\boldsymbol{\Phi}$, which vanishes in this normal phase.

DMFT instead is able to capture the effect of coherent hopping processes, resulting in a further broadening of the resonances. This finite hopping correction to the spectral function reflects the fact that the stationary density matrix in the normal phase is not a tensor product of single-site density matrices, as predicted by Gutzwiller, but rather includes correlations among neighboring sites encoded within DMFT in the non-Markovian bath.

A comparison between Hubbard-I and NCA shows that the former largely underestimates the effect of the bath. Indeed, within NCA the sharp peaks of the isolated singlesite problem are largely broadened already for a moderate value of the hopping rate $J / U=0.2$, a trend that further increases for larger values of $J / U$. At the same time, the location of the poles is found to be weakly dependent on the hopping rate and, at least for $J / U=0.2$, essentially captured already by Hubbard-I and Gutzwiller mean-field theory. This difference can be understood by noticing that there are two main sources of resonance broadening within our DMFT approach, one coming from the bare nonMarkovian bath $\Delta(\omega)$ and the other coming from the Markovian interacting single-site problem, encoded in the self-energy $\Sigma(\omega)$ (see Sec. III C). Within Hubbard-I, the latter is independent of $J$ and set only by pump and losses. NCA, on the other hand, accounts for many-body scattering channels mediated by the bath and results in an imaginary part of $\Sigma(\omega)$, also scaling with the hopping strength and responsible for the larger broadening. We emphasize that, while the main effect of the DMFT bath in this regime is to broaden the resonances, this broadening is not uniform in frequency; i.e., it could not be reproduced by treating the DMFT bath with a Markov approximation. In fact, the self-consistent condition Eq. (13) implies that the spectrum of the DMFT bath is given by the local spectral function itself; namely, it comes with a rich multipeak structure in frequency which prevents the use of a simple Markovian approximation. Overall, the spectral function in this low-drive, low-hopping normal region is very reminiscent of an equilibrium Bose-Hubbard model in the Mott insulating phase [127], with Hubbard bands, describing doublons and holons and multiparticle excitations, which are partially filled by incoherent pump and dissipation. As we show in the next section, increasing the pump strength reveals a spectral feature which is instead unique to interacting driven-dissipative systems.

\section{Negative density of states}

We now discuss how the spectral features of the normal phase evolve upon increasing the strength of the drive/loss ratio $r$. While in the low pump regime all the peaks of the spectral function are positive (see Fig. 5), a novel effect appears at large drives. Above a threshold $r_{\mathrm{NDOS}}$, the lowest 


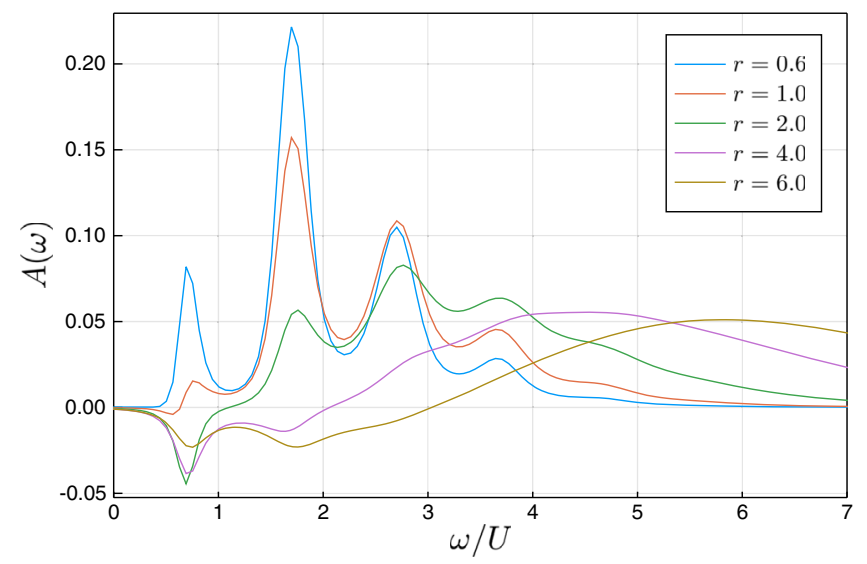

FIG. 6. Local spectral function $A(\omega)$ obtained from DMFT NCA for different values of the pump/loss ratio $r$ at fixed $J / U=0.2$ and $z=6$. Upon increasing $r$, the lowest Hubbard band flips sign and a region of NDOS emerge at positive frequencies, up to $\omega=\Omega_{0}(r)$, where the spectral function vanishes, $A\left(\Omega_{0}\right)=0$. The spectral range of NDOS increases with $r$. Parameters: $\operatorname{dim}_{H}=14$.

Hubbard band flips sign and a region of NDOS appears in a positive frequency range [139].

We show this region in Fig. 6, where we plot the spectral function obtained within DMFT+NCA for different values of drive/loss ratio $r$, at fixed $J / U=0.2$. The region of NDOS extends up to $\omega=\Omega_{0}$, a frequency at which the imaginary part of the retarded Green's function linearly vanishes; i.e., we have

$$
A(\omega)=\gamma\left(\omega-\Omega_{0}\right) \quad \text { for } \omega \simeq \Omega_{0}
$$

with $\gamma>0$, while for $\omega>\Omega_{0}$ the conventional positive sign is recovered. As we show in Fig. 6, the spectral range of NDOS increases with the drive $r$, and so does the frequency $\Omega_{0}(r)$. We stress that a negative spectral function at positive frequency is a genuine nonequilibrium phenomenon that cannot happen for closed systems in thermal equilibrium [113]. It has direct physical consequences on the response of the system to a weak local coherent drive oscillating at frequency $\omega, V(t)=\sum_{i}\left[v_{i}^{*}(t) b_{i}+\right.$ H.c. $]$ with $v_{i}^{*}(t)=v_{0} \delta_{i, 0} e^{i \omega t}$. Indeed, for an open system, the power absorbed from the perturbation, defined as [140-142] $\dot{W}=\operatorname{Tr} \rho(t) \dot{V}$, can be written within linear response theory as (see Appendix D)

$$
\dot{W}=v_{0}^{2} \omega A(\omega) .
$$

This expression highlights how the spectral function at frequency $\omega$ controls the power absorbed by the system under an external drive. A change in sign of this quantity, i.e., a negative absorbed power, signals the onset of energy emission and gain, a condition which is generally associated with optical amplification and lasing [143-146]. As we discuss in Sec. VC, the NDOS effect and the frequency $\Omega_{0}$ play a crucial role in the nonequilibrium phase transition from the normal to the superfluid phase.

We emphasize that the NDOS effect arises already in the single-site problem, i.e., for $J=0$ in our model, above a threshold pump $r_{\mathrm{NDOS}}$ which depends on the strength of Kerr nonlinearity, as discussed in Ref. [113]. As a result, it naturally appears at large drive in the normal-phase spectral function of our lattice model calculated within Gutzwiller mean-field theory as well as DMFT+Hubbard-I, both built out of the exact solution of the single-site problem.

We now discuss the dependence of the NDOS effect from the hopping $J$. Clearly, such a question goes beyond Gutzwiller mean-field theory, which as we stress cannot capture any effect due to coherent hopping within the normal phase. In Fig. 7, we plot the spectral function obtained with DMFT+Hubbard-I and NCA, for increasing values of $J / U$, and compare with the results obtained from Gutzwiller. We find that the NCA spectral function is strongly affected by the hopping, which broadens the sharp high-energy peaks and decreases the strength of the negative peak around $\Omega_{0}$, up to a value of $J / U \simeq 0.8$, at which this peak turns back to positive, washing away the NDOS effect. In other words, NCA is able to capture a renormalization of the scale $\Omega_{0}$ from the hopping $J$. This result is surprising at first, since $J$ is a purely coherent energy scale, while we seen in Fig. 6 that the strength of the peaks and the NDOS is controlled by the dissipative scales, i.e., the pump-to-loss ratio. We interpret this effect as a first example of hopping-induced losses, a mechanism that is unique to open quantum systems and that plays a key role in the physics of our model. Importantly, this effect is

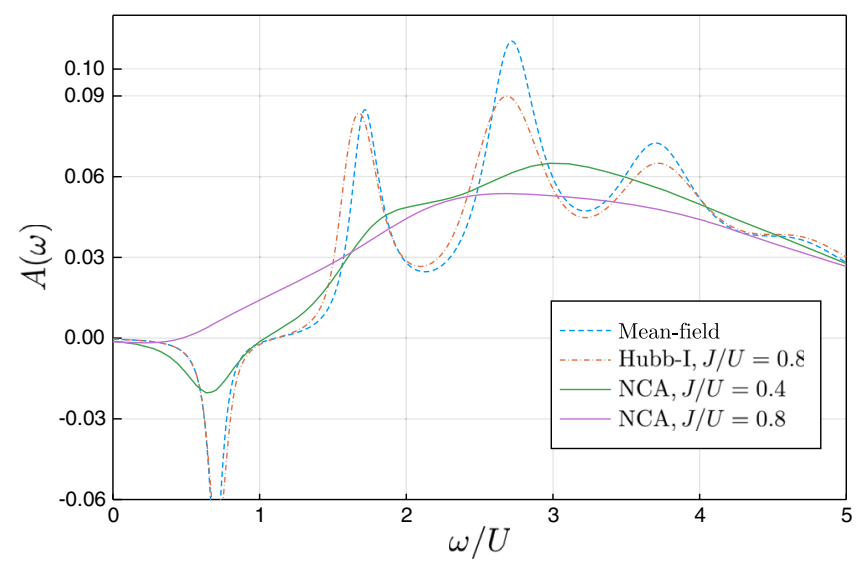

FIG. 7. Local spectral function $A(\omega)$ for different values of $J / U$, as computed within Gutzwiller mean-field theory (dashed blue line), DMFT with Hubbard-I impurity solver (dashed red line), and NCA impurity solver (green and pink full lines), for fixed $z=6$ and $r=2$. Within NCA, we see that increasing the hopping $J$ changes qualitatively the structure of the lowfrequency spectrum, washing out the NDOS and restoring a positive spectrum at $\omega>0$. This effect of the DMFT bath is completely missed by Gutzwiller mean-field as well as Hubbard-I approximations. Parameters: $\operatorname{dim}_{H}=10$. 
completely missed by the simple impurity solver Hubbard-I, whose spectral function, also shown in Fig. 7, changes very little with respect to the Gutzwiller mean-field one [147].

In summary, we see that changing drive and hopping largely affects the spectral properties of the normal phase. In particular, we identify for positive frequencies $0<\omega<$ $\Omega_{0}(r, J / U)$ a region of NDOS emerging above a threshold drive strength $r>r_{\mathrm{NDOS}}(J / U)$. Both these quantities depend within DMFT NCA from the hopping-to-interaction ratio $J / U$, an effect which is completely missed by Gutzwiller mean-field theory as well as by Hubbard-I. As we discuss in Sec. VC, these dependencies of the critical frequency $\Omega_{0}$ and of the threshold drive $r_{\mathrm{NDOS}}$ from the hopping strength $J$ have direct consequences on the phase diagram of the model.

\section{B. Steady-state local density matrix and population inversion}

We now discuss the occupation properties of the stationary-state distribution in the normal phase. For a lattice problem, computing the full many-body density matrix can be done only for very small systems. Nevertheless, within our DMFT NCA approach, describing the thermodynamic limit of infinitely many sites, we can compute the reduced steady-state density matrix of a given site of the lattice, say, site $i=0$, obtained by performing a partial trace on all other sites, namely, $\rho_{s}=\operatorname{tr}_{j \neq 0} \rho_{\text {latt }, s}$. This result corresponds to the steady-state density matrix of the DMFT selfconsistent quantum impurity model (9) and, thus, of the non-Markovian map $\hat{\mathcal{V}}$ (25), and it obeys Eq. (31). This reduced on-site stationary density matrix allows us to study the change of the local populations of bosons due to hopping processes, which is completely missed by Gutzwiller mean-field theory. Also, for open systems, these hopping processes enable new, effective dissipative channels. For example, a particle can be injected from the Markovian environment on one site, hop to another site, and escape the system rather than just being created and annihilated on the same site. Those processes are captured by our DMFT approach and mimicked by the nonMarkovian environment $\Delta$ and unlock interesting new physics which we discuss here.

\section{Local occupation versus J}

From the knowledge of the on-site reduced stationarystate density matrix $\rho_{s}$, we can obtain the average local density $\langle n\rangle=\operatorname{tr}\left(b^{\dagger} b \rho_{s}\right)$. We notice that the local density can be also obtained from the Green's functions, in particular, from the Keldysh component at equal times:

$G^{K}(t, t)=-i\left\langle\left\{b(t), b^{\dagger}(t)\right\}\right\rangle=-i\left[2\left\langle b^{\dagger}(t) b(t)\right\rangle+1\right]$,

which gives consistently the same result in our NCA approach.

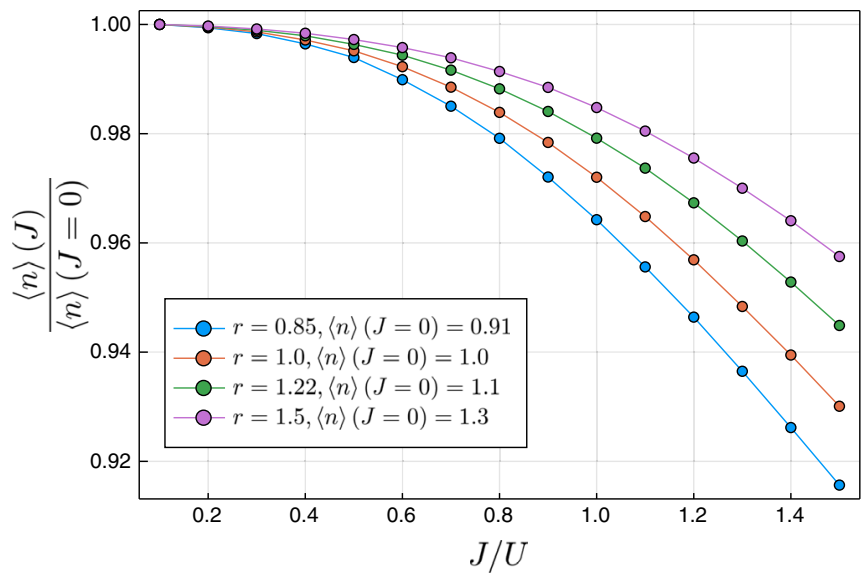

FIG. 8. Local density of particles as a function of the hoppingto-interaction ratio $J / U$, within DMFT NCA, for different values of the pump-to-loss ratio $r$, normalized to the values of the singlesite problem. We see that the density decreases with $J / U$, a specific feature of driven-dissipative lattices with two-body losses (see the main text), which is captured by DMFT. Parameters: $z=50$ and $\operatorname{dim}_{H}=10$. The drive values used are marked on the $y$ axis in Fig. 10.

Within DMFT, the local density acquires a dependence from the hopping $J$, which is obviously missing in Gutzwiller mean-field theory. In Fig. 8, we plot the density as a function of $J / U$ at $z=6$ and for different values of the drive, normalized to the mean-field value $(z=\infty)$. We see that quite generically the density decreases smoothly upon increasing the hopping within the normal phase, i.e., for $J<J_{c}$. This result can be understood as an interplay of twoparticle losses and coherent hopping between neighboring sites, an effect that is further explained by discussing the stationary-state populations in the next section. Interestingly, the rate of decrease of the density with hopping changes quite strongly with the strength of the pump $r$, and, in particular, we notice in Fig. 8 that a large drive seems to make the density more pinned to the single-site value.

The result in Fig. 8 turns out to be a specific feature of dissipative lattices with two-particle losses. In fact, one can generically prove that, for a driven-dissipative BoseHubbard model with only single-particle losses and single-particle drive, the stationary-state density matrix is independent of any Hamiltonian parameter [148], leading to a density of particles independent of $J$ (although not necessarily integer, as it would be in the equilibrium Mott ground state of the Bose-Hubbard model) and set only by drive-loss balance. In Appendix B, we show that this effect is correctly captured by our DMFT NCA approach, a highly nontrivial benchmark for its validity.

\section{Steady-state populations and population inversion}

In this section, we discuss the effect of coherent hopping processes on the steady-state reduced density matrix, which as we show exhibits richer physics than the local 


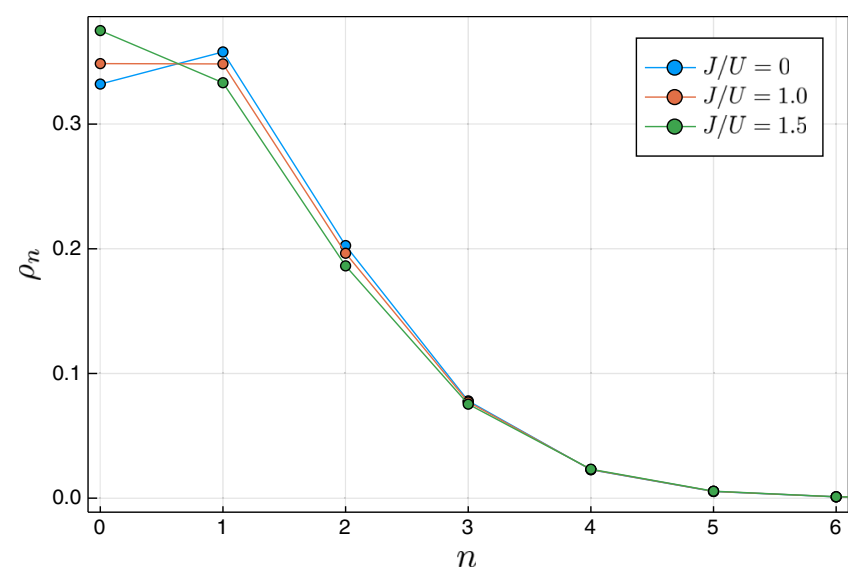

FIG. 9. Steady-state populations of the reduced density matrix $\rho_{n}=\left\langle n\left|\rho_{s}\right| n\right\rangle$, within DMFT NCA, for different values of the hopping-to-interaction ratio $J / U$ and for drive-to-loss ratio $r=1.22$. We see that increasing $J / U$ changes the populations at low values of $n$ and ultimately washes away the population inversion found in the single-site limit $J / U=0$. Parameters: $z=$ 50 and $\operatorname{dim}_{H}=10$. The drive-to-loss ratio value used is marked on the $y$ axis in Fig. 10.

occupancy. In the normal phase, this quantity is diagonal in the basis of Fock states with $n$ bosons per site, and the steady-state populations $\rho_{n}$ are shown in Fig. 9 for different hopping values and for $z=6$ and $r=3$.

First, we observe that the single-site model, corresponding to $J=0$, shows a nonmonotonic behavior of the populations as a function of the number of bosons per site, for drive/loss ratio $r>1$ and any value of the Kerr nonlinearity $U$ (which, in fact, does not affect the stationary state as has been long known [111]). This population inversion at $J=0$ appears clearly in Fig. 9, where the probability of finding $n$ bosons per site is maximum at $n=1$ despite the fact that a finite bosonic occupation costs energy $E_{n}=\omega_{0} n+U n^{2} / 2 \sim U$ and should be, therefore, thermodynamically suppressed.

Increasing the hopping changes the populations at low occupancy while leaving essentially unaffected the tail at large $n$. In particular, the coherent hopping from and into the neighboring sites increases the probability of having an empty site at the expense of finite occupation. This increase is a genuine feature of our dissipative many-body lattice problem with local two-body losses: Starting from a state with average filling $n \sim 1$, hopping processes toward neighboring sites create double occupations which escape at a rate $\eta$, reducing the total occupation. This trend goes on upon further increasing $J$, ultimately suppressing the population inversion above a threshold hopping. This mechanism also explains more in detail the observed overall decrease of average occupation with $J$ (Fig. 8), which we discuss in the previous section.

An interesting question concerns the relation between the NDOS effect discussed in Sec. VA and the population inversion in the reduced stationary density matrix. In closed quantum systems described by unitary evolution, the two concepts are directly related; namely, an NDOS could emerge only in the presence of an inversion of populations where higher-energy states are more occupied than lowerenergy ones. For open quantum systems, the situation is more subtle, and the two concepts are not in one-to-one correspondence [113]. In Fig. 10, we plot the behavior of the threshold for population inversion $r_{\text {inv }}$ and for NDOS $r_{\mathrm{NDOS}}$ as a function of $J / U$. We notice that those thresholds are independent from the hopping within Gutzwiller meanfield theory (see dashed lines, which coincide with the $J=0$ values of DMFT), while they are substantially renormalized in DMFT. In particular, the two scales $r_{\text {NDOS }}<r_{\text {inv }}$ further deviate from each other as the hopping is increased. We note that $r_{\text {inv }}$ increases monotonically with the hopping strength $J$ in DMFT. Based on closed system arguments, this hopping-induced suppression of population inversion would suggest that the NDOS is also always suppressed by hopping, as, for example, Fig. 7 shows. Surprisingly, this suppression is not always the case. Figure 10 shows that $r_{\text {NDOS }}$ has a nonmonotonic behavior with the hopping rate $J$; namely, its behavior changes from small and large hopping values. While for large values of $J$ the NDOS threshold $r_{\mathrm{NDOS}}$ indeed increases following the behavior of the $r_{\text {inv }}$ threshold, as expected from closed system arguments, for small values of $J$ it is actually reduced below the $J=0$ threshold, corresponding to the single site. Namely, for small values of $J$, the nonMarkovian bath $\Delta$ actually generates an NDOS, even in a regime where the single-site model would not present any

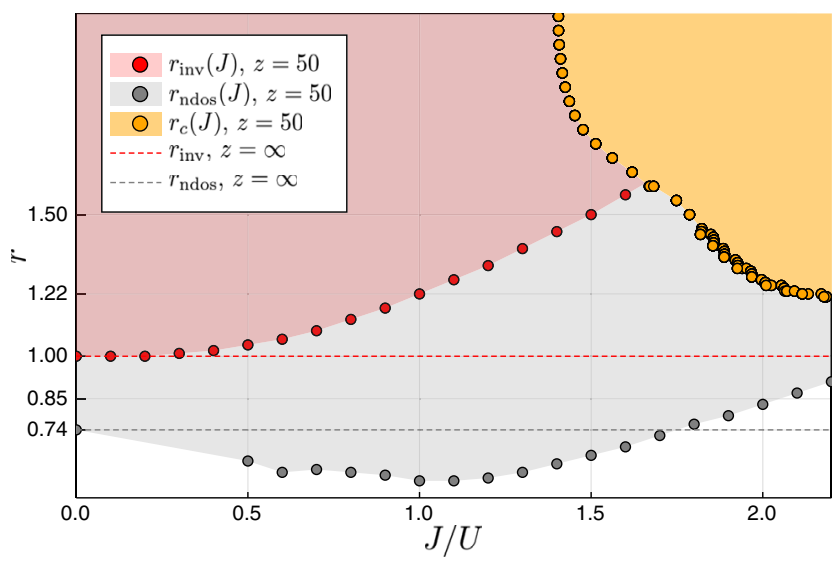

FIG. 10. Threshold drives for NDOS $r_{\text {NDOS }}(J)$ and for population inversion in the density matrix $r_{\text {inv }}(J)$, as a function of the hopping and compared with the critical drive $r_{c}(J)$ (yellow points) for the finite-frequency superfluid transition. The ticks of the $y$ axis correspond to the values of $r$ used for Figs. 8 and 9. We see that for a small value of $r$ the phase transition occurs even in the absence of a population inversion; i.e., it is the NDOS the key effect leading to the instability of the normal phase. Parameters: $z=50$. Red curve: $t_{\max }=10$ and $\operatorname{dim}_{H}=14$. Gray curve: $t_{\text {max }}=20$ and $\operatorname{dim}_{H}=10$. 
signature of this effect. This result is a unique feature of dissipative quantum systems, where an NDOS can be generated even in the absence of population inversion. For Markovian systems, it is shown that this feature can be traced back to the structure of excitations on top of the stationary state, which come with characteristic complex weights, leading to anti-Lorentzian line shapes [113].

\section{Finite-frequency instability of the normal phase}

In this section, we discuss how the peculiar spectral and occupation properties of the normal phase contribute to an instability toward a spontaneous breaking of $\mathrm{U}(1)$ symmetry. We show that the conventional static superfluid transition of the equilibrium Bose Hubbard model as a function of the hopping-to-interaction ratio $J / U$ is pushed to finite frequency as a result of drive and dissipation, leading to an order parameter oscillating in time. We emphasize the role of the NDOS for the onset of the phase transition and compare the DMFT NCA and Gutzwiller phase boundaries. We argue that the effect of finiteconnectivity fluctuations not only is quantitative, but rather underlines a qualitatively new physical mechanism for the onset of an ordered phase in open quantum lattices with two-body losses, which cannot be simply interpreted as the destruction of an ordered phase by thermal fluctuations in an effective equilibrium problem.

\section{DMFT phase boundary}

Within our DMFT approach, we can derive an equation for the phase boundary separating the normal and the broken symmetry phases. We assume to be in the early symmetry-broken phase, where the order parameter $\boldsymbol{\Phi}(t)=$ $\langle\mathbf{b}(t)\rangle$ has just formed and it is small. This assumption implies a small external field $\boldsymbol{\Phi}_{\text {eff }}(t)$ (11) in the DMFT effective action (9). We also assume to be in a stationary regime at long times, such that two-point correlators depend only on time differences and move to Fourier space. The average value of the bosonic field $\boldsymbol{\Phi}(\omega) \equiv$ $\langle\mathbf{b}(\omega)\rangle$ is, to linear order in $\boldsymbol{\Phi}_{\text {eff }}$,

$$
\boldsymbol{\Phi}(\omega)=-\mathbf{G}^{R}(\omega) \boldsymbol{\Phi}_{\mathrm{eff}}(\omega),
$$

where we use the fact that $\langle\mathbf{b}(\omega)\rangle_{\boldsymbol{\Phi}_{\text {eff }}=0}=0$. A key point now is that at finite $z$ the effective field $\boldsymbol{\Phi}_{\text {eff }}(\omega)$ in DMFT has two contributions, one from the local order parameter itself and the other from neighboring sites encoded in the non-Markovian bath; see Eq. (11), which now reads (using $\boldsymbol{\Phi}_{+}=\boldsymbol{\Phi}_{-}$as well as $\left.\boldsymbol{\Phi}_{\text {eff }+}=\boldsymbol{\Phi}_{\text {eff }-}\right)$

$$
\boldsymbol{\Phi}_{\mathrm{eff}}(\omega)=J \boldsymbol{\Phi}(\omega)+\boldsymbol{\Delta}^{R}(\omega) \boldsymbol{\Phi}(\omega) .
$$

Plugging Eq. (38) into Eq. (37) and using the DMFT selfconsistency on the Bethe lattice (13), one finally gets

$$
\boldsymbol{\Phi}(\omega)=\left(-J \mathbf{G}^{R}(\omega)-\frac{J^{2}}{z} \mathbf{G}^{R}(\omega) \mathbf{G}^{R}(\omega)\right) \boldsymbol{\Phi}(\omega) .
$$

The critical coupling $J_{c}$ and critical frequency $\Omega_{c}$ needed for a self-consistent broken-symmetry solution, $\boldsymbol{\Phi}\left(\Omega_{c}\right) \neq 0$ corresponding to an order parameter whose phase oscillates in time $\langle b(t)\rangle \sim e^{-i \Omega_{c} t}$ for $J>J_{c}$, are given by

$$
\frac{1}{J_{c}}+G^{R}\left(\Omega_{c}, J_{c}\right)+\frac{J_{c}}{z}\left[G^{R}\left(\Omega_{c}, J_{c}\right)\right]^{2}=0 .
$$

Equation (40), which to the best of our knowledge is an original result of this paper, is generic for bosonic DMFT theories on the Bethe lattice, and it holds also for equilibrium problems. Its solution, leading to the phase boundary in Figs. 2 and 10, strongly depends on the drivendissipative nature of the problem, as we discuss now. First, Eq. (40) has real and imaginary parts, which both need to vanish simultaneously, resulting in the two conditions

$$
\begin{gathered}
\operatorname{Im} G^{R}\left(\Omega_{c}, J_{c}\right)=0, \\
\frac{1}{J_{c}}+\operatorname{Re} G^{R}\left(\Omega_{c}, J_{c}\right)+\frac{J_{c}}{z}\left[\operatorname{Re} G^{R}\left(\Omega_{c}, J_{c}\right)\right]^{2}=0 .
\end{gathered}
$$

We remark that there is another solution possible, where $\operatorname{Im} G^{R}\left(\Omega_{c}, J_{c}\right) \neq 0$, but this result is never realized in our simulations. In thermal equilibrium, the first condition Eq. (41) can be satisfied only at zero frequency, where fluctuation-dissipation theorem constrains the imaginary part of a bosonic retarded Green's function to vanish, thus allowing for static symmetry-breaking patterns (as in equilibrium superfluids). Far from equilibrium, this situation does not need to be the case $[109,149]$, and indeed we see that the normal phase shows, above a threshold drive $r_{\mathrm{NDOS}}$, a spectral function vanishing at a positive frequency, corresponding to the formation of an NDOS and the onset of gain in the system. The critical frequency $\Omega_{c}$ solving Eq. (41) corresponds, therefore, to the frequency at which the local spectral function of the normal phase vanishes:

$$
\Omega_{c}=\Omega_{0}\left[r, J_{c}(r)\right]
$$

for a critical value of hopping $J_{c}$ determined by jointly solving Eq. (42). The energy scale $\Omega_{0}$ for the NDOS is, therefore, a precursor of the mode that becomes unstable at the transition. We conclude that the NDOS effect discussed in the previous section is a key, necessary condition for a phase transition into the nonequilibrium superfluid phase. This transition is clearly shown in Fig. 10, where we plot the threshold pump $r_{\mathrm{NDOS}}$ for NDOS and the critical drive $r_{c}$ obtained from solving Eq. (40) with DMFT NCA as a function of $J / U$. We see that generically $r_{\mathrm{NDOS}}<r_{c}$; namely, the system first develops gain and then becomes truly unstable toward $\mathrm{U}(1)$ symmetry breaking. On the 
other hand, from Fig. 10, we see that one can obtain an instability even in the absence of population inversion.

\section{Role of finite-connectivity fluctuations and comparison with Gutzwiller}

We now go back to an important aspect mentioned at the beginning of our paper, namely, the large renormalization to the phase boundary obtained within DMFT NCA upon decreasing the connectivity $z$. As we show in Figs. 2 and 11, fluctuations due to the finite number of neighbors shift the phase boundary toward larger values of the hopping $J$ and pump/loss ratio $r$. We now provide a physical interpretation of this effect based on the properties of the normal phase discussed so far.

We start by considering the condition for the normal phase instability obtained within Gutzwiller mean-field theory, which corresponds to the $z \rightarrow \infty$ limit of Eq. (40). In this limit, the problem reduces to a quantum single site, while the feedback from neighboring sites is treated at a purely classical level (see Sec. III A), in terms of a selfconsistent coherent field which reads $\Phi_{\text {eff }}=J_{c}\langle\mathbf{b}(t)\rangle \sim$ $J e^{i \Omega_{c} t}$ near the instability. As such, if we repeat the argument of the previous section, we can obtain a condition for the Gutzwiller phase boundary, which reads

$$
\frac{1}{J_{c}(z=\infty)}+G_{0}^{R}\left[\Omega_{c}(z=\infty)\right]=0,
$$

where the first term is the effective field contribution and $G_{0}^{R}(\omega)$ is the retarded Green's function of the isolated



FIG. 11. Phase diagram in the pump versus hopping plane obtained by DMFT NCA for $z=50$ (yellow points) and Gutzwiller mean-field theory $(z=\infty$, yellow dashed line). We further plot the thresholds for NDOS obtained within the two approaches. We see that fluctuations due to finite connectivity reduce the broken symmetry phase, pushing it toward higher values of hopping and drive. We interpret this effect as a signature of hopping-induced losses, which reduce the local gain and prevent the system from becoming unstable at finite frequency. Parameters: $\operatorname{dim}_{H}=10$. single-site problem and is, therefore, independent from the hopping. Within Gutzwiller mean-field theory, the hopping $J$ has only the role of triggering the symmetry breaking, through the self-consistent field $\Phi_{\text {eff }}$, while the onset of gain is controlled by the pump-to-loss ratio $r$. Indeed, we know that the single-site spectral function develops an NDOS above a constant threshold pump $r_{\text {NDOS }}$ (see the gray dashed line in Fig. 11). The feedback from the neighboring sites acts as a seed for a single site which is on the verge of energy emission [negative absorbed power at $\Omega_{c}$; see Eq. (35)] and leads, above a threshold hopping $J_{c}$ shown in Fig. 11, to amplification of the local coherent field at frequency $\Omega_{c}$ and a spontaneous breaking of $\mathrm{U}(1)$ and time-translation symmetry. Interestingly, the Gutzwiller phase boundary is very close to the line $r_{\mathrm{NDOS}}$ (see Fig. 11), suggesting that at large hopping as soon as the system develops gain the symmetry breaking occurs.

DMFT NCA, on the other hand, accounts for a more subtle effect of neighboring sites, which are encoded in the non-Markovian quantum bath, in addition to the classical coherent field. As we know, this effect provides an increased effective dissipation, due to hopping processes from lossy neighboring sites, which is responsible for wiping out the NDOS region in the local spectral function, a necessary condition for the onset of the instability. This result is clearly shown in Fig. 10, where for large values of $J / U$ the threshold for NDOS eventually becomes larger than the Gutzwiller mean-field one (see the gray dashed line in Fig. 10), leaving a normal phase which would be superfluid at $z=\infty$. These hopping-induced losses provide, therefore, a clear physical mechanism behind the finite-connectivity renormalization of the phase boundary. The picture that emerges from DMFT is one of a single site on the verge of energy emission, coupled to an oscillating seed field which would favor optical amplification and embedded in a non-Markovian bath which is instead able to absorb part of the emitted power from the system, thus reducing the effective gain and requiring a stronger value of pump to trigger the instability. This mechanism is presumably also effective at an intermediate value of the hopping where, as we see in Fig. 11, the DMFT NCA threshold for NDOS remains well below the critical drive responsible for the true many-body instability at intermediate coupling while approaching it at large hopping.

We conclude that this hopping-induced dissipation is a qualitatively new mechanism for the destruction of an ordered phase, which is unique to open systems settings and one of the hallmarks of our DMFT NCA approach. Quite interestingly, this mechanism is completely missed not only by the Gutzwiller mean-field approach, but also by a perturbative solver such as the Hubbard-I approximation. In fact, we discuss (see Fig. 7) how the NDOS is changed very little within this scheme. In Appendix E, we also show that the Hubbard-I phase diagram, obtained using the DMFT equation for the critical point (40), still reduces 


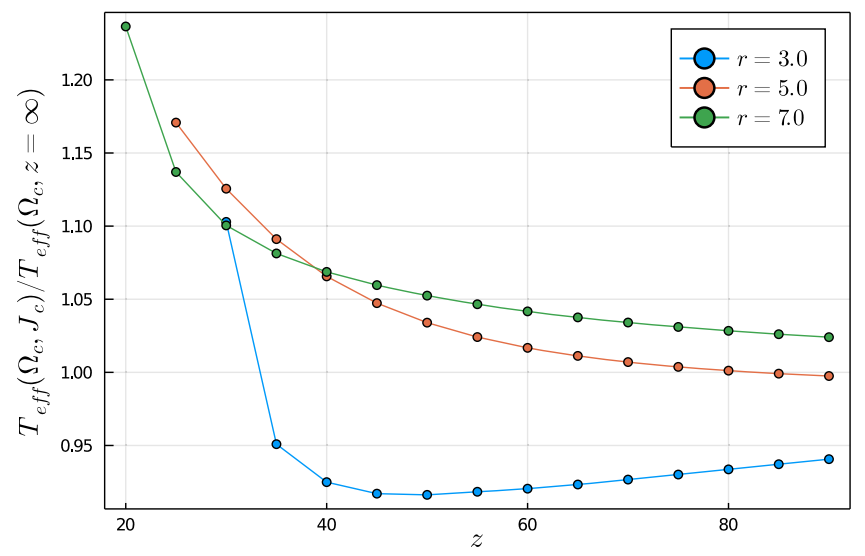

FIG. 12. Change of the effective temperature $T_{\text {eff }}$ at the critical point $\left(\Omega_{c}, J_{c}\right)$ as a function of lattice connectivity $z$, with respect to its mean-field value at $z=\infty$, for different values of the pumpto-loss ratio $r$. We see that the relative change in $T_{\text {eff }}$ remains of the order of a few percent up to connectivity $z=50$ and slightly increases up to $20 \%$ for the lowest connectivity $z=20$. This result should be compared to the DMFT renormalization of the critical hopping for similar values of drive and connectivity, which is instead much stronger as shown in Fig. 2. In particular, for $z=30$ and $r=3$ the critical hopping is pushed to infinity corresponding to a complete destruction of the normal phase. Parameters: $\operatorname{dim}_{H}=14$.

to the Gutzwiller mean-field one. This result further highlights the nonperturbative nature of the fluctuations responsible for the observed renormalization of the NDOS and the importance of using a self-consistent scheme such as our NCA approach.

\section{Thermal versus nonthermal origin of finite-connectivity fluctuations}

A different perspective on the role of finite-connectivity fluctuations on the phase boundary can be obtained by looking at the occupation of single-particle modes at finite frequency, describing excitations on top of the stationary state and their effective thermal character. In fact, in an effective equilibrium picture, one could expect heating to provide an efficient mechanism for the reduction of a broken symmetry phase. To investigate this physics, we look at the Keldysh Green's function, defined in Eq. (36), which heuristically describes the fluctuations of the observable $b$. If the system was in true thermal equilibrium, the quantum fluctuation-dissipation theorem (FDT) would constrain the Keldysh and the retarded components to obey the relation [126]

$$
\frac{G^{K}(\omega)}{-2 \pi i A(\omega)} \equiv F_{\mathrm{eq}}(\omega)=\operatorname{coth}\left(\frac{\omega}{2 T}\right),
$$

where $T$ is the system temperature. At low frequency or high temperatures, $\omega \ll T$, one has $F_{\text {eq }}(\omega) \sim T / \omega$. In a nonequilibrium system, on the contrary, there is no well-defined temperature and the FDT does not hold, in general. Nonetheless, it is useful to use the left-hand side of the FDT relation in Eq. (45) to define an effective distribution function

$$
F_{\text {neq }}(\omega)=\frac{i G^{K}(\omega)}{2 \pi A(\omega)}
$$

and to study its frequency dependence. Within the DMFT and Gutzwiller normal phases, for pump above the threshold for NDOS $r>r_{\text {NDOS }}$, the spectral function $A(\omega)$ vanishes at frequency $\Omega_{0}$, with linear corrections [see Eq. (34)]. On the other hand, we find that the Keldysh component has a finite nonzero value at $\Omega_{0}$, which gives a distribution function of pseudoequilibrium form at least for the modes around $\Omega_{0}$ :

$$
F_{\text {neq }}(\omega) \simeq \frac{T_{\text {eff }}}{\omega-\Omega_{0}} .
$$

From this expression, we can therefore identify an effective temperature $T_{\text {eff }}$, which emerges quite ubiquitously in nonequilibrium quantum systems [52,150-154]. At $z=\infty$, when the normal phase is described as a collection of independent sites, an effective temperature emerges due to the interplay of local drive and Kerr interaction [113]. Within DMFT NCA, one could, in principle, expect important corrections due to the non-Markovian bath, as we see for the NDOS. To assess this point, we sit at the phase boundary $J_{c}(r)$, choose the corresponding critical frequency $\Omega_{0}\left(J_{c}, r\right)=\Omega_{c}$, and plot in Fig. 12 the behavior of $T_{\text {eff }}$, scaled with respect to the $z=\infty$ value, as a function of the lattice connectivity $z$ and for different values of the pump-to-loss ratio $r$. We see that at large values of $r$ the effective temperature slightly increases upon decreasing $z$, while at smaller pumps, i.e., $r=3$, it shows a weak nonmonotonic behavior with $z$. Overall, the relative variation of $T_{\text {eff }}$ with respect to the Gutzwiller mean-field value remains rather moderate. On the other hand, in the same range of variation of $z$, the DMFT critical hopping shows instead strong renormalizations. This result is particularly true for the small drive regime, where already for $r=3$ and $z=30$ the ordered phase is completely washed out. This observation suggests that effective thermal fluctuations and heating are not enough to explain the large renormalization of the phase diagram observed in DMFT, which is instead mainly driven by the reduction of local gain by virtual hopping processes, through the mechanism of hoppinginduced losses.

\section{Nonequilibrium superfluidity, lasing, and many-body synchronization of Van der Pol arrays}

We now discuss how the nonequilibrium superfluid transition in our Bose-Hubbard model is connected to other dynamical phenomena associated with breaking of 
time-translation symmetry, such as nonequilibrium BoseEinstein condensation, lasing, and synchronization of Van der Pol oscillators. To appreciate this point, it is useful to start from the semiclassical limit of our model (see Appendix C), where nonlinear quantum fields contributions are disregarded. The dynamics of the bosonic field at site $j$ takes the form of a Langevin equation:

$i \dot{b}_{j \mathrm{cl}}=\left[\tilde{\omega}_{0}\left(b_{j \mathrm{cl}}\right)+i \gamma\left(b_{j \mathrm{cl}}\right)\right] b_{j \mathrm{cl}}+\frac{J}{z} \sum_{\left\langle j^{\prime}\right\rangle} b_{j^{\prime} \mathrm{cl}}+\xi_{j}(t)=0$

with an effective frequency and damping terms which depend nonlinearly on the field itself, i.e., $\tilde{\omega}_{0}\left(b_{j \mathrm{jl}}\right)=\omega_{0}+$ $U\left|b_{j \mathrm{cl}}\right|^{2} / 2$ and $\gamma\left(b_{j \mathrm{cl}}\right)=f / 2-\eta\left|b_{j \mathrm{cl}}\right|^{2} / 2$, and where $\xi_{j}(t)$ is a zero average white noise $\left\langle\xi_{i}(t) \xi_{j}\left(t^{\prime}\right)\right\rangle=f \delta\left(t-t^{\prime}\right) \delta_{i j}$. In the continuum limit, this form reduces to a complex Gross-Pitaevskii equation $[155,156]$ with pump and nonlinear losses which describes a variety of nonlinear phenomena from exciton polariton condensates to multimode lasers [157]. In the absence of any noise, the spatially uniform stationary state admits a stable limit cycle, i.e., $\beta(t)=|\beta| e^{-i \omega_{\mathrm{vdp}} t}$ for $r>0$ and any $J$ with frequency $\omega_{\mathrm{vdp}}$ :

$$
\omega_{\mathrm{vdp}}=\omega_{0}-J+U|\beta|^{2} / 2
$$

and amplitude set by the incoherent drive, $|\beta|=\sqrt{r}$. Equation (48) is, therefore, the semiclassical version of our condition for finite-frequency instability in the normal phase. A major difference exists between the lasing threshold and our case, namely, that the threshold for the onset of gain and the one for development of full coherence is well separated, while it coincides in the usual lasing regime [149]. This difference can be seen easily by looking at the Green's function which develops an NDOS as soon as the system becomes unstable.

The equation above describe also an array of coupled classical Van der Pol oscillators. Our driven-dissipative Bose-Hubbard model can be, therefore, also seen as a quantum many-body version of the VdP array. From this perspective, the onset of finite-frequency oscillations at $J_{c}(r)$ described in the last section can be seen as a signature of a quantum synchronization [70-82], where above a certain coupling $J$ all quantum $\mathrm{VdP}$ oscillators enter into a collective limit-cycle phase. As we discuss next, these oscillations share qualitative features with the semiclassical solution at least at large drive values, while they deviate significantly for smaller drives where quantum fluctuations are important.

Despite the similarities, the semiclassical limit described above is rather different from the large connectivity limit: In the former case, one has a deterministic nonlinear equation, and desynchronization can appear only due to the noise. In the latter, instead, corresponding to DMFT, one reduces to a quantum VdP oscillator coupled to a self-consistent field and a quantum bath. This difference is responsible for the large separation between the onset of gain and the true instability.

\section{Critical frequency versus drive/loss ratio and Kerr nonlinearity}

In this section, we discuss the behavior of the critical frequency $\Omega_{c}$, signaling the onset of a quantum synchronized phase, as a function of pump/loss ratio $r$ and Kerr nonlinearity $U$. In Fig. 13, we plot this frequency as a function of the incoherent drive amplitude $r$, both for DMFT (for $z=50$ ) and for Gutzwiller mean-field theory $(z=\infty)$. We see that $\Omega_{c}$ scales linearly with the drive at large values of $r$ (see the fit in Fig. 13), a result which is in agreement with the semiclassical result obtained for the VdP array Eq. (48) where $\omega_{\mathrm{vdp}} / U \sim r / 2$.

As the pump is reduced and the number of bosons per site decreases, one expects quantum fluctuations to become more important. Indeed, we see significant deviations from the semiclassical result at small $r$, already captured by Gutzwiller mean-field theory but more pronounced for DMFT. Another interesting aspect is the role played by the Kerr nonlinearity. In Fig. 14, we plot the local density of states for a fixed value of hopping and drive/loss ratio and for different values of the interaction $U$. A first interesting observation is that the frequency $\Omega_{0}$ at which NDOS emerges, related to the mode becoming critical at the synchronization transition [see Eq. (43)], decreases upon reducing the Kerr nonlinearity. A detailed analysis shows $\Omega_{c} \sim U$. We notice the analogy with the semiclassical result (48); nevertheless, we stress that in this picture the

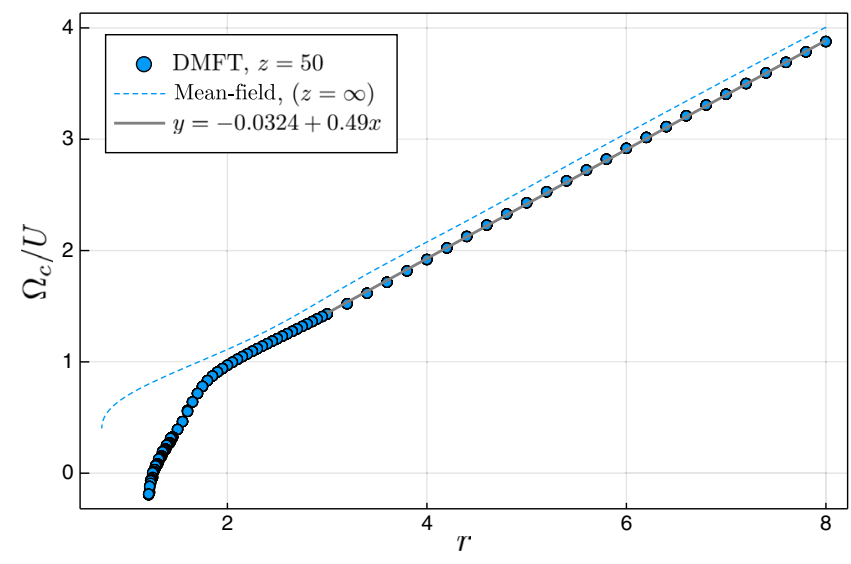

FIG. 13. Critical frequency for quantum synchronization $\Omega_{c}$ as a function of the pump/loss ratio $r$, within DMFT NCA for connectivity $z=50$ and Gutzwiller mean-field theory $(z=\infty)$. In the region of large drive, the fit shows that $\Omega_{c} / U \sim r / 2$, in agreement with the semiclassical result for the limit cycle in the $\mathrm{VdP}$ array. At lower drives, in the regimes of few bosons per site, quantum fluctuations become relevant and the critical frequency is strongly renormalized. Parameters: $\operatorname{dim}_{H}=14$. 




FIG. 14. Local spectral function $A(\omega)$ for increasing values of the Kerr nonlinearity $U$, at a fixed value of hopping $J=\omega_{0}$ and drive-to-loss ratio $r=3$ within DMFT NCA. We see that the frequency $\Omega_{0}$ at which the density of states vanishes decreases with $U$ and eventually disappears for small enough values of $U$. Parameters: $z=6$ and $\operatorname{dim}_{H}=14$.

critical frequency is proportional to the modulus square of the order parameter $|\langle b\rangle|^{2} \neq 0$, while within DMFT we find $\Omega_{c} \sim U$ at the critical point, where by definition $\langle b\rangle=0$. This result further highlights the quantum nature of the synchronization transition considered in this work. Indeed, we see how $\Omega_{c}$ is smoothly connected to the frequency $\Omega_{0}$ where NDOS emerges, a scale that exists already well inside the normal incoherent phase and that is a genuine feature of the quantum impurity model. Furthermore, this result implies that the Kerr nonlinearity is crucial in order to push the transition at finite frequency, implying undamped oscillations of the order parameter. We indeed observe that, for a related model of all-to-all coupled quantum VdP oscillators, recent Gutzwiller mean-field analysis reports a static (first-order) transition in the absence of any Kerr nonlinearity $[70,119]$.

We conclude by noting that an oscillating phase in the order parameter could be, in principle, gauged away by going to an appropriate rotating frame. This gauging, however, could not be done a priori, since as we see the critical frequency $\Omega_{c}$ itself depends on the many-body physics of the problem. We are considering here only the instability of the normal phase rather than the dynamics in the full broken symmetry phase which could show a more complex dynamical behavior, whose description goes beyond the scope of the stationary-state-oriented approach (see Sec. IV B 1) used here.

\section{Limit cycles at finite connectivity}

Limit cycles emerge ubiquitously within mean-field approaches [38,158-160], and, indeed, even in the present problem the Gutzwiller solution at $z=\infty$ predicts one. The role of fluctuations on their stability has been discussed before, in particular, in the context of a coherently driven anisotropic Heisenberg model with spontaneous decay [161]. There, an approach based on self-consistent Mori projection [162] and cluster mean field [51] predicts that limit cycles disappear as the coordination number $z$ is decreased below a threshold value $z^{*}$, which depends on the system parameters.

On the basis of these results, it is particularly interesting to study the fate of our synchronization transition beyond Gutzwiller mean-field theory. In Fig. 15, we plot the behavior of the critical frequency $\Omega_{c}$ obtained from DMFT NCA, as a function of $z$ for different values of $r$. We observe that finite $z$ corrections tend to reduce the value of $\Omega_{c}$ with respect to the mean-field value, which nevertheless remains finite down to the lowest value of connectivity at which, for a given value of drive, a synchronization transition exists, consistently with the phase boundary moving to higher values of $r$ for decreasing $z$ (see Fig. 2). In other words, we find that, within our treatment, finite connectivity does not destroy the limit cycle phase which is pushed only at higher values of the incoherent drive. Indeed, in Fig. 15, we show that, for drive $r=7$, the highest value that we can numerically access given the constraints on the local Hilbert space truncation, a limit cycle exists down to $z=20$. We, therefore, expect that at higher drives the synchronized phase would survive down to low connectivity values. The regime of strong drive is, however, difficult to access within NCA, and we leave this question open for future works. We remark, nevertheless, that our model is different from the one of Ref. [161]. In the present case, the existence of a limit cycle phase is tightly related to a U(1) symmetry present in the original Lindblad problem and spontaneously broken at the transition [109].

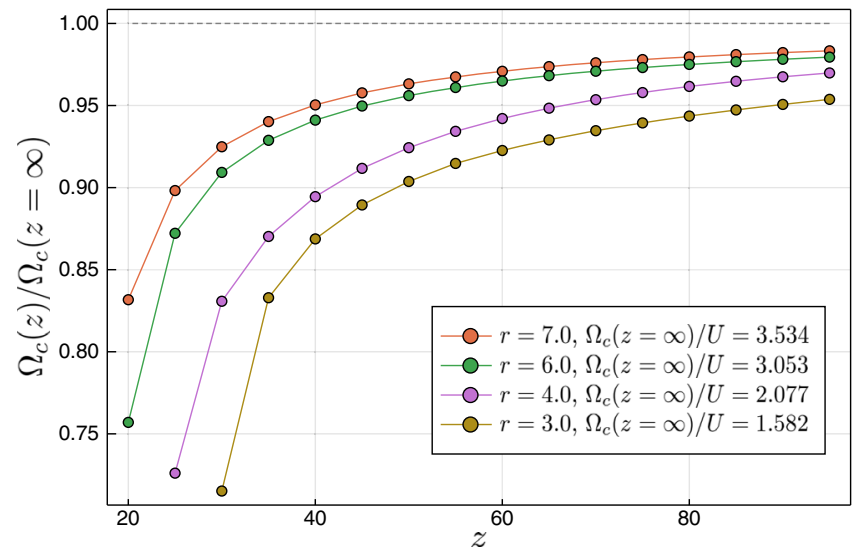

FIG. 15. Critical frequency for quantum synchronization, $\Omega_{c}$, within DMFT NCA, as a function of the lattice connectivity $z$, for different values of the drive-to-loss ratio $r$ and hopping fixed on the phase boundary $J / U=(J / U)_{c}$. For clarity, we normalize $\Omega_{c}$ to the mean-field value obtained for $z=\infty$. We see that decreasing $z$ renormalizes down the frequency but does not destroy the limit cycle. Parameters: $\operatorname{dim}_{H}=14$. 


\section{E. Quantum-Zeno regime}

In the previous sections, we see that the key feature of DMFT is the ability to capture hopping-induced dissipative processes in the normal phase, which are missed by Gutzwiller mean-field theory and ultimately responsible for the large corrections to the phase boundary due to finite connectivity. In this section, we discuss a different parameter regime of our model, corresponding to large twoparticle losses $\eta \gg J$, which allows us to highlight even more the qualitative difference between DMFT and Gutzwiller predictions. In the large two-particle-losses limit, and in the absence of any external drive, a perturbative analysis shows that the dissipative dynamics of the Bose-Hubbard model effectively takes place in the subspace with zero and one boson per site, which are dark states of the local dissipator [120]. These emergent hardcore bosons are subject to next-neighbor losses controlled by the scale

$$
\Gamma_{\text {eff }}=\frac{2(J / z)^{2}}{U^{2}+\eta^{2}} \eta
$$

This effective dissipation is hopping mediated-namely, it is nonzero only at finite hopping $J$-and, remarkably, it shows a nonmonotonic behavior as a function of the physical dissipation $\eta$ : For $\eta \ll U$, it increases linearly with $\eta$. Instead, when $\eta \gg U$, the effective dissipation $\Gamma_{\text {eff }}$ is suppressed by increasing the physical dissipation $\eta$. The latter is a quantum-Zeno regime [121,122], in which the coherent hopping dynamics bringing the system outside of the hard-core bosons subspace is suppressed due to the large coupling to the environment [120]. In the absence of an external pump, the effective loss rate (49) ultimately leads the system to a zero density state, and the Zeno scale (49) manifests itself in the transient dynamics $[120,123]$. Here, instead, we demonstrate how, in the presence of a small pump, the quantum-Zeno regime emerges in the stationary-state properties of the system and how this effect is completely missed by Gutzwiller, even at a qualitative level, while captured by our DMFT NCA approach.

We consider the regime of $\eta \gg J$ and a parametrically small pump rate $f \ll \eta$. In this case, we expect the local occupation of all the states with more than one boson per site to be largely suppressed upon increasing the twoparticle losses, since the small residual pump is not sufficient to counterbalance the losses. This result is indeed shown in Fig. 16, where we plot the occupation probabilities of the on-site reduced density matrix obtained from DMFT NCA and see that only the occupation of the states $n=0,1$ remain of the order of one, while $\rho_{n \geq 1}$ is exponentially suppressed. We emphasize that in this case the long-time limit of the problem remains nontrivial within this subspace, due to the interplay between the small residual pump and the coherent dynamics generated by the Hamiltonian. In Fig. 3, we show that our DMFT NCA



FIG. 16. Quantum-Zeno regime. Occupation probabilities of the DMFT NCA on-site reduced density matrix $\rho_{n}=\left\langle n\left|\rho_{s}\right| n\right\rangle$, as a function of the number of particles per site and for different values of two-particle-loss rates $\eta / J$ and for $z=6, J / U=0.133$ and $U=15$. For large $\eta / J$, we see that probabilities $\rho_{n \geq 2}$ are suppressed exponentially (note the log scale), with a rate that increases with $\eta / J$, while the states with $n=0,1$ bosons, not affected by the losses, retain a population of the order of one.

approach is able to capture the emergence of a stationary Zeno regime, through a nonmonotonic behavior of the steady-state probability of having exactly one bosons per site, namely, $\rho_{1}$, versus $\eta / U$, showing a universal collapse when plotted against $\Gamma_{\text {eff }}$. This result is remarkable, since the dissipative scale in Eq. (49) describes particle losses which are nonlocal in space [120] which a priori could have been expected to be beyond reach for a local approach such as DMFT. Instead, the non-Markovian bath is able to capture this hopping-induced dissipative process. We emphasize that DMFT goes beyond the effective hard-core model in Ref. [120] in that the full crossover from normal to Zeno phase is captured as $\eta / J$ is increased. In this sense, we can see DMFT as providing a nonperturbative solution of the effective hard-core model in the limit of large lattice connectivity.

The presence of Zeno scale $\Gamma_{\text {eff }}$ in the system not only affects the steady-state populations, but is also expected to influence the lifetime of excitations of the steady state, which can be extracted from the Green functions computed in DMFT. In Fig. 17(a), we show the behaviour of the spectral function, defined in Eq. (33), for increasing $\eta / J$. We work in a regime in which $U$ is large so that different resonances in the spectrum, corresponding to singleparticle transitions between states with occupation $n$ and $n+1$, are well separated [113]. By increasing $\eta / J$, all the peaks but the first one decrease in amplitude, as the former are proportional to the occupation of states with $n \geq 2$, which decreases with $\eta$ (see Fig. 16). The amplitude of the lowest-frequency peak, instead, increases with $\eta$ due to the increased probability of being in the subspace with 
(a)

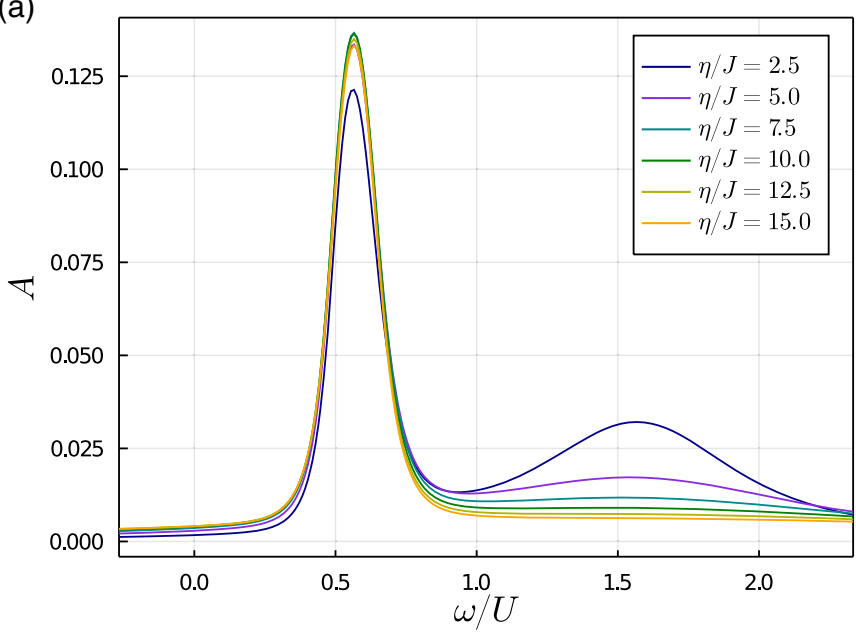



(c)

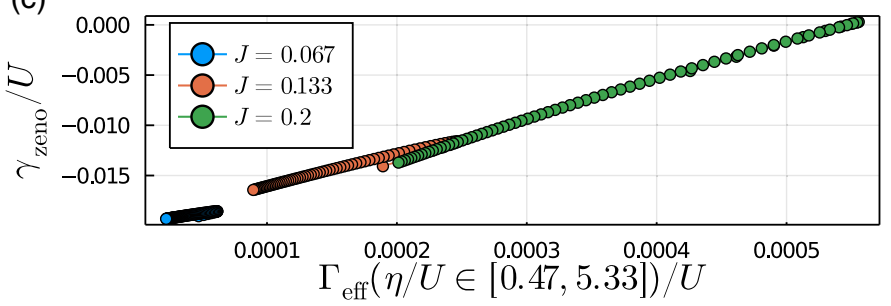

FIG. 17. Quantum-Zeno regime in the spectral function, increasing two-particle-loss rate $\eta$ at fixed drive rate $f / U=r \eta / U=0.013$ and for $z=6, U=15$. Parameters: $t_{\max }=20, d t=0.002$, and $\operatorname{dim}_{H}=6$. (a) Spectral function at increasing $\eta / J$ and for $J / U=0.133$. The first peak corresponds to transitions between states with on-site occupation $n=0$ and $n=1$, while higher-energy peaks are suppressed at large $\eta / J$. We fit the first peak as described in the main text in order to extract a measure of its lifetime $\gamma$. (b) Partial lifetime $\gamma_{\text {Zeno }} / U$ of the first peak of the spectral function obtained by removing the zero hopping and zero drive or dissipation contributions. We show that this quantity has a nonmonotonic behavior, analogous to the populations in Fig. 3, manifesting a quantum-Zeno behavior in the lifetime of excitations of the steady state. (c) $\gamma_{\text {Zeno }} / U$ depends only on the dissipative scale $\Gamma_{\text {eff }} / U$, analogously to the occupation probability $\rho_{1}$ as shown in the inset in Fig. 3. In (b), (c) $J / U=[0.067,0.133,0.2]$.

occupation $n=0,1$. We concentrate on this peak in order to extract its width. In order to do so, we fit the peak with a generalized Lorentzian $A(\omega)=a\left[\left(\omega-\omega_{0}\right) b+\right.$ $1] /\left[\left(\omega-\omega_{0}\right)^{2}+\gamma^{2}\right]$, with fitting parameters $a, b$, and $\gamma$, which exactly describes the shape of the peak at zero hopping and which approximates it at small hopping, whose lifetime is given by the fitting parameter $\gamma$.

We remark that the width $\gamma$ of the first peak has three contributions: $\gamma=\gamma_{\text {diss }}+\gamma_{\text {coh }}+\gamma_{\text {Zeno. }}$. The first term comes from dissipation and pump at zero hopping $\gamma_{\text {diss }}=\gamma(J=0, \eta, f, U)$, and it can be well estimated with perturbation theory [113], which shows it does not depend on $\eta$, as the transition $n=0 \rightarrow n=1$ is unaffected by the two-particle losses at $J=0$. The second contribution to the lifetime is given by the hybridization of the energy levels of different sites at finite hopping, which is a purely coherent effect, independent of drive and dissipation. For example, this effect is responsible for the formation of the holon and doublon bands in the local spectral functions of the isolated Bose-Hubbard model; see, e.g., Ref. [127]. We call this term $\gamma_{\text {coh }}=\gamma(J, \eta=0, f=0, U)$.

Finally, we expect a contribution coming from the hopping-induced decay, which we call $\gamma_{\text {zeno }}$, which is present only for finite hopping and dissipation or drive. In Fig. 17(b), we show that the latter contribution follows a similar nonmonotonic behavior as $\Gamma_{\text {eff }}$ as a function of $\eta / U$, witnessing the presence of quantum-Zeno behavior in steady-state excitations. Analogously to populations, in Fig. 17 (c), we show that, plotting $\gamma_{\text {Zeno }} / U$ as a function of $\Gamma_{\text {eff }} / U$, the data points at different values of $J / U$ all collapse on the same curve, demonstrating that $\gamma_{\text {Zeno }}$ depends on $J$ and $\eta$ only through $\Gamma_{\text {eff }}$.

\section{CONCLUSIONS}

In this paper, we formulate the dynamical mean-field theory for bosonic open quantum many-body systems described by a Lindblad master equation on the lattice. This method is based on a systematic expansion in the limit of large lattice connectivity $z$. Within DMFT, fluctuations due to finite lattice connectivity are treated nonperturbatively through the solution of a self-consistent quantum impurity model, which in our case amounts to a Markovian single-site problem coupled to a coherent field and to a nonMarkovian quantum bath mimicking the rest of the lattice. The non-Markovian bath contains the key new ingredient of DMFT, which makes it different from the Gutzwiller mean-field theory, to which it reduces in the $z=\infty$ limit. In particular, with respect to the former, DMFT is able to capture both coherent and dissipative processes arising from the neighboring sites, the former playing a particularly crucial role in open quantum systems with correlated jump operators.

Using DMFT, together with a nonperturbative bosonic impurity solver based on a superoperator hybridization expansion truncated at the NCA level, we solve a BoseHubbard model in the presence of two-particle losses and single-particle pump, relevant for dissipative ultracold atoms as well as for arrays of superconducting circuits. We show that this model features a dissipative phase 
transition from an incoherent normal phase to a nonequilibrium superfluid, which occurs above a critical hopping or pump strength.

Within DMFT NCA, the phase boundary is strongly renormalized with respect to the Gutzwiller one and pushed toward higher values of the couplings, leading to an increase of the normal phase. We identify a new mechanism for this reduction of the broken symmetry phase which is unique to open quantum systems and arises from the suppression of local gain due to hopping-induced losses. This mechanism is a key feature brought forth by NCA, which treats the non-Markovian DMFT bath nonperturbatively, and it is instead missed by a simpler perturbative DMFT solver based on the Hubbard-I approximation. We further discuss how the increased effective dissipation due to the finite number of lossy neighbors affects all the unusual properties of the DMFT NCA normal phase, from the renormalization of the gain (NDOS) threshold and steady-state populations due to hopping to the emergence of a stationary-state quantum-Zeno regime for large twobody losses. These effects are all qualitatively missed by Gutzwiller mean-field theory.

Finally, we show that the transition into the nonequilibrium superfluid phase occurs at finite frequency, corresponding to a local order parameter that oscillates in time at a frequency $\Omega_{*}$. Within DMFT NCA, this scale depends on both coherent and dissipative couplings, another important aspect which is missed by Gutzwiller mean-field theory. Drawing from the physics of quantum VdP oscillators, we interpret this phenomenon as the onset of quantum manybody synchronization and limit cycles at finite lattice connectivity.

Our DMFT holds the promise to be applied to a variety of driven-dissipative quantum many-body problems. Different bosonic models or driving schemes can be considered and readily studied with the NCA approach developed here, such as the recently introduced quadratically driven Kerr resonator [40], the coherently driven case explored in the context of quantum bistability $[47,54,105$, $107,108]$, or models relevant for the dissipatively stabilized Mott insulators of photons [16]. Interesting directions for the future involve the development of other quantum impurity solvers, made possible by the Markovian structure of the quantum impurity problem. In particular, one could go beyond the noncrossing approximation by including higher-order diagrams or resumming the full hybridization expansion [69] with real-time diagrammatic Monte Carlo methods $[130,132]$. Alternatively, one could map back the non-Markovian Keldysh action into a Lindbladian problem with a finite number of bath levels [86] which could then be solved by exact diagonalization [163], quantum trajectories, through a matrix product operator representations of the density matrix [164,165], or using an extension of the numerical renormalization group [166]. Further extensions of this approach could include the development of a cluster version of DMFT building upon recent developments for Markovian problems [51].

Finally, we notice that a similar DMFT NCA impurity solver could be developed for driven-dissipative fermionic problems [67,167] or quantum spins [168], which are also being actively investigated and relevant for different experimental platforms [169-173].

\section{ACKNOWLEDGMENTS}

This work was supported by the University of Chicago through a FACCTS grant ("France and Chicago Collaborating in The Sciences"), by the CNRS through the PICS-USA-147504, by a grant "Investissements d'Avenir" from LabEx PALM (ANR-10-LABX-0039PALM), and from the ANR grant "NonEQuMat" (ANR19-CE47-0001). A. A. C. acknowledges support from the Air Force Office of Scientific Research MURI program, under Grant No. FA9550-19-1-0399, and from a Simons Foundation Simons Investigator Award (Award No. 669487, AC). R. F. acknowledges partial financial support from the Google Quantum Research Award. R. F. research has been conducted within the framework of the Trieste Institute for Theoretical Quantum Technologies (TQT).

\section{APPENDIX A: DERIVING DMFT FOR OPEN MARKOVIAN QUANTUM SYSTEMS}

We present here a sketch of the derivation of the DMFT action and self-consistency conditions, using the quantum cavity method and following and extending Ref. [68] to the open case. The main idea is to single out a given site of the lattice, $i=0$ in the following, and to write down its effective Keldysh action obtained after integrating out all the other sites:

$$
i \mathcal{S}_{\mathrm{eff}}\left[\bar{b}_{0}, b_{0}\right]=\log \int \prod_{i \neq 0} D\left[\bar{b}_{i}, b_{i}\right] e^{i \mathcal{S}} .
$$

This effective single-site action describes, in principle, all the effects on site $i=0$ due to the coupling to the other sites by the hopping $J$, which, for our assumption of local jump operators and interactions (Sec. II), is the only term responsible for the coupling between different sites. To proceed, we notice that the full action Eq. (7) can be divided into three terms:

$$
\mathcal{S}=\mathcal{S}_{0}+\delta \mathcal{S}+\mathcal{S}_{\mathrm{cav}}^{(0)}
$$

respectively, $\mathcal{S}_{0}=\mathcal{S}\left[\bar{b}_{0}, b_{0}\right]$ containing only the terms in Eqs. (7) and (8) involving fields at site $i=0$, a term $\delta \mathcal{S}$ containing the hopping terms to the $2 z$ fields $\left(\bar{b}_{j}, b_{j}\right)$ at the neighboring sites

$$
\delta \mathcal{S}=\frac{J}{z} \int d t \sum_{\alpha} \alpha \sum_{\langle 0 j\rangle}\left(\bar{b}_{0 \alpha} b_{j \alpha}+\text { H.c. }\right),
$$




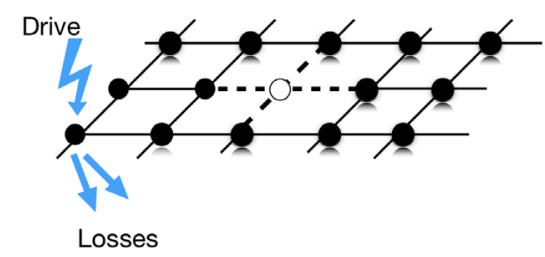

FIG. 18. Sketch of the quantum cavity method to derive the effective action of DMFT for Markovian lattice problems (see the text). A given lattice site is singled out (white dot in the figure), and all the remaining degrees of freedom are integrated out. Because of the coupling between the site and its $2 z$ neighbors (dashed lines), this integration of degrees of freedom reduces to evaluating correlation function of a lattice with a cavity, containing all the remaining sites.

and a term $\mathcal{S}_{\text {cav }}^{(0)}$ describing a lattice with a cavity, namely, including all the degrees of freedom except those at site $i=0$; see Fig. 18 . For an interacting many-body problem on a finite-dimensional lattice, integrating over the neighboring sites can be done only formally, as a cumulant expansion in $\delta S$, and leads to an effective action containing arbitrary powers of the local fields $\bar{b}_{0}$ and $b_{0}$, with coefficients given by the multipoint correlation functions of the cavity problem [62]. In the large connectivity limit $z \gg 1$, one can formally organize this expansion in power of $1 / z$ and obtain

$$
\begin{aligned}
\mathcal{S}_{\mathrm{eff}}\left[\mathbf{b}_{0 \alpha}^{\dagger}, \mathbf{b}_{0 \alpha}\right]= & S_{0}+\int d t \sum_{\alpha= \pm} \alpha \boldsymbol{\Phi}_{\mathrm{eff} \alpha}^{\dagger}(t) \mathbf{b}_{0 \alpha}(t) \\
& +\frac{i^{2}}{2} \int d t d t \sum_{\alpha, \beta= \pm} \alpha \beta \mathbf{b}_{0 \alpha}^{\dagger}(t) \Delta^{\alpha \beta}\left(t, t^{\prime}\right) \mathbf{b}_{0 \beta}\left(t^{\prime}\right),
\end{aligned}
$$

where, in order to allow for condensed phases, we introduce the Nambu-spinor notation

$$
\mathbf{b}_{0}^{\dagger}=\left(b_{0} \bar{b}_{0}\right), \quad \mathbf{b}_{0}=\left(\begin{array}{c}
b_{0} \\
\bar{b}_{0}
\end{array}\right) .
$$

The coefficients entering the effective action (A3) are related to quantum averages over the cavity action $\mathcal{S}_{\text {cav }}^{(0)}$ :

$$
\begin{aligned}
\boldsymbol{\Phi}_{\mathrm{eff} \alpha}^{\dagger}(t) & =J / z \sum_{\langle j 0\rangle}\left\langle\mathbf{b}_{j \alpha}^{\dagger}(t)\right\rangle_{\mathrm{cav}}^{(0)}, \\
\boldsymbol{\Delta}^{\alpha \beta}\left(t, t^{\prime}\right) & =J^{2} / z^{2} \sum_{\langle j 0\rangle\langle k 0\rangle}\left\langle\mathbf{b}_{k \alpha}(t) \mathbf{b}_{j \beta}^{\dagger}\left(t^{\prime}\right)\right\rangle_{\mathrm{cav}}^{(0)} .
\end{aligned}
$$

The final step, in order to obtain the self-consistent conditions, is to relate the average of bosonic fields on the cavity action to those evaluated to the effective action (A3). For what concerns the non-Markovian bath in Eq. (A5), this relation depends on the lattice geometry: It becomes particularly transparent for a Bethe lattice, a lattice with no loops such that, once a cavity is created, two neighbors $j$ and $k$ are completely disconnected, where one gets

$$
\begin{aligned}
\left\langle\mathbf{b}_{k \alpha}(t) \mathbf{b}_{j \beta}^{\dagger}\left(t^{\prime}\right)\right\rangle_{\mathrm{cav}}^{(0)} & =\delta_{k j}\left\langle\mathbf{b}_{j \alpha}(t) \mathbf{b}_{k \beta}^{\dagger}\left(t^{\prime}\right)\right\rangle_{\mathrm{cav}}^{(0)} \\
& =\delta_{k j}\left\langle\mathbf{b}_{j \alpha}(t) \mathbf{b}_{j \beta}^{\dagger}\left(t^{\prime}\right)\right\rangle_{S} \\
& =\delta_{k j}\left\langle\mathbf{b}_{0 \alpha}(t) \mathbf{b}_{0 \beta}^{\dagger}\left(t^{\prime}\right)\right\rangle_{S_{\mathrm{eff}}},
\end{aligned}
$$

where we use the property of the Bethe lattice in the first equality, the fact that in the thermodynamic limit the local property of the cavity action and the original action must be the same in the second equality, and translational invariance in the last step. Plugging this equation into Eq. (A5) gives the self-consistency condition (13). We notice that similar arguments can be used for a different lattice, to relate averages over cavity and effective action; the only difference would be a more complicated self-consistency relation between the bath and local Green's function [62]. Finally, the average of the bosonic field taken on the cavity action can be related to the one on the effective action (A3) as [68]

$$
\begin{aligned}
\left\langle\mathbf{b}_{j \alpha}^{\dagger}\right\rangle_{\mathrm{cav}}^{(0)} & =\left\langle\mathbf{b}_{0 \alpha}^{\dagger}\right\rangle_{S_{\mathrm{eff}}} \\
& +\int d t^{\prime} \sum_{\beta= \pm} \beta\left\langle\mathbf{b}_{0 \beta}^{\dagger}\left(t^{\prime}\right)\right\rangle_{S_{\mathrm{eff}}}\left\langle\mathbf{b}_{k \beta}\left(t^{\prime}\right) \mathbf{b}_{j \alpha}^{\dagger}(t)\right\rangle_{\mathrm{cav}}^{(0)}
\end{aligned}
$$

which can be plugged in Eq. (A4) to give the second selfconsistent condition (11).

\section{APPENDIX B: NCA BENCHMARK: LOCAL OCCUPATION WITH SINGLE-PARTICLE LOSSES}

In this Appendix, we report a benchmark our DMFT NCA approach for driven-dissipative many-body master equations defined by Eqs. (2) and (3). We consider a different model from the main text, namely, the drivendissipative Bose-Hubbard model with single-particle losses and single-particle drive. This model is specified by the same Bose-Hubbard Hamiltonian Eqs. (3) and (4) as in the main text, but with the jump operators

$$
\begin{gathered}
\hat{L}_{i, l}=\sqrt{\kappa} \hat{b}_{i}, \\
\hat{L}_{i, p}=\sqrt{f} \hat{b}_{i}^{\dagger},
\end{gathered}
$$

where there are single-particle losses instead of the twoparticle losses of the main text.

One can prove [148] that the stationary-state density matrix of this model is independent from any Hamiltonian parameter; thus, for example, the on-site occupation is constant with the hopping rate $J$. Obtaining this constant occupation with $J$ is a highly nontrivial benchmark for our DMFT NCA approach. Figure 19 shows that this behavior is correctly reproduced by our approach. Small 


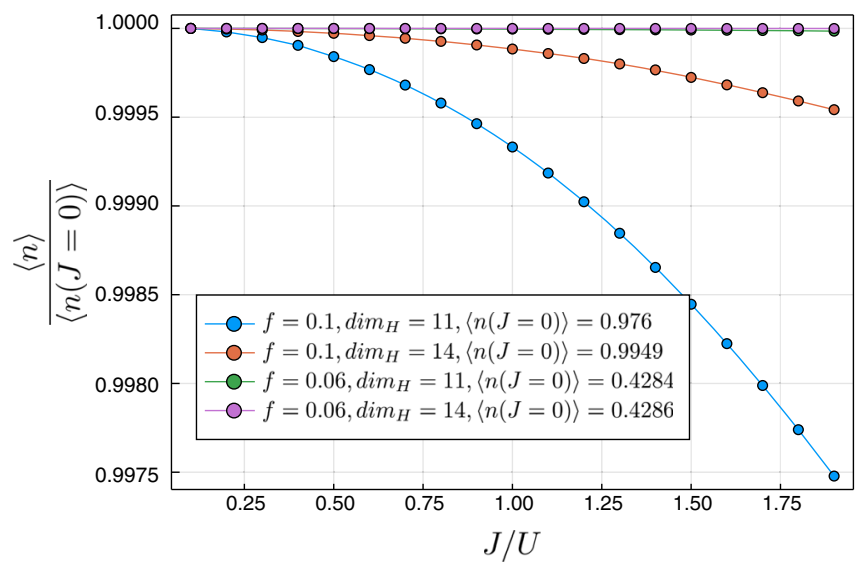

FIG. 19. Local density of particles for the BH model with single-particle losses, as a function of the hopping-to-interaction ratio $J / U$, for different values of the drive amplitude $f$, normalized to the values of the single-site problem. Parameters: $z=30, \kappa=0.2$, and $d t=0.005$.

deviations from constant occupation show that this property is not exactly enforced by our numerical scheme and, thus, is a good test to validate our approach. Deviations from constant occupation are mostly a result of local Hilbert space truncation, which become more severe increasing the drive, but they are reduced by increasing the cutoff $\operatorname{dim}_{H}$.

\section{APPENDIX C: KELDYSH FIELD THEORY AND SEMICLASSICAL LIMIT}

In this Appendix, we write down the Keldysh field theory associated to the Lindblad master equation for our driven-dissipative Bose-Hubbard model in Eqs. (3)-(5), which is done by writing a coherent path-integral representation of the trace over the density matrix $[52,126]$ :

$$
Z=\operatorname{Tr} \rho(t)=\int \prod_{i} D \bar{b}_{\mathrm{icl}} D b_{\mathrm{icl}} D \bar{b}_{i q} D b_{i q} \exp (i \mathcal{S})
$$

in terms of bosonic fields in the basis of classical and quantum fields $b_{j \mathrm{cl} / q}=\left(b_{j+} \pm b_{j-}\right) / \sqrt{2}$, where the Keldysh action reads

$$
\begin{aligned}
S= & \int d t \sum_{j}\left[\bar{b}_{j q}\left(i \partial_{t}-\omega_{0}-i \chi_{-}\right) b_{j \mathrm{cl}}+\text { H.c. }\right] \\
& -\frac{1}{2} \int d t \sum_{j}\left[(U+i \eta)\left(\bar{b}_{j \mathrm{cl}}^{2} b_{j \mathrm{cl}} b_{j q}+\bar{b}_{j q}^{2} b_{j \mathrm{cl}} b_{j q}\right)+\text { H.c. }\right]
\end{aligned}
$$

$$
\begin{aligned}
& +\int d t \sum_{j}\left(2 i \chi_{+}-i \eta\left|b_{j \mathrm{cl}}\right|^{2}\right) \bar{b}_{j q} b_{j q} \\
& -\frac{J}{z} \int d t \sum_{\left\langle j j^{\prime}\right\rangle}\left(\bar{b}_{j q} b_{j / \mathrm{cl}}+\text { H.c. }\right),
\end{aligned}
$$

where the first line describes the local noninteracting contribution, including the dissipative couplings $\chi_{ \pm}=\left(\gamma_{p} \pm \gamma_{l}\right) / 2$ which in the case considered in the main text, where $\gamma_{l}=0$ and $\gamma_{p}=f=r \eta$, reduce to $\chi_{ \pm}=f / 2$. The second line includes nonlinearities due to interaction and two-body losses, while the last term accounts for the hopping between neighboring sites. The semiclassical limit is obtained by disregarding terms with more than two quantum fields, which leaves the action at most quadratic in the quantum fields $b_{j q}$ [52]. Those quadratic terms are decoupled using a classical stochastic field [126], which allows one to write the action as

$$
\begin{aligned}
S= & \int d t \sum_{j}\left\{\bar{b}_{j q}\left[i \partial_{t}-\omega_{0}-i \chi_{-}+\xi_{j}(t)\right] b_{j \mathrm{cl}}+\text { H.c. }\right\} \\
& -(U-i \eta) \int d t \sum_{j} \bar{b}_{j q}\left|b_{j \mathrm{cl}}\right|^{2} b_{j \mathrm{cl}}+\text { H.c. } \\
& -\frac{J}{z} \int d t \sum_{\left\langle j j^{\prime}\right\rangle}\left(\bar{b}_{j q} b_{j^{\prime} \mathrm{cl}}+\text { H.c. }\right) .
\end{aligned}
$$

We can now take the saddle point conditions

$$
\frac{\delta S}{\delta \bar{b}_{j q}}=0=\frac{\delta S}{\delta \bar{b}_{j \mathrm{cl}}}
$$

and obtain the Langevin equation given in Eq. (47) of the main text. We note that the noninteracting retarded Green's function of the problem reads

$$
G_{0}^{R}(\omega)=\frac{1}{\omega-\omega_{0}-i \chi_{-}},
$$

which has NDOS as soon as $\chi_{-} \geq 0$, i.e., $r>0$, which is in the condition for the instability toward an oscillating solution.

\section{APPENDIX D: NDOS AND RESPONSE TO A WEAK COHERENT DRIVE}

In this Appendix, we show that a direct consequence of the NDOS effect is that the average power absorbed from a weak coherent drive becomes negative, indicating the onset of gain and energy emission. We consider our lattice model in the presence of a time-dependent perturbation of the Hamiltonian describing a weak coherent drive with frequency $\omega$ :

$H(t)=H+V(t) \equiv H+\sum_{i}\left(v_{i}^{*} e^{i \omega t} b_{i}+v_{i} e^{-i \omega t} b_{i}^{\dagger}\right)$,

where $H$ is the Bose-Hubbard Hamiltonian defined in Eqs. (3) and (4). The evolution of the density matrix of the system is described by the Lindblad master equation in the presence of $H(t)$ : 
$\partial_{t} \hat{\rho}=-i[H(t), \hat{\rho}]+\sum_{i \mu}\left(\hat{L}_{i \mu} \hat{\rho} \hat{L}_{i \mu}^{\dagger}-\frac{1}{2}\left\{\hat{L}_{i \mu}^{\dagger} \hat{L}_{i \mu}, \hat{\rho}\right\}\right)$.

We notice that the time derivative of the internal energy $E(t)=\langle H(t)\rangle$ in an open quantum system has two contributions, respectively coming from the derivative of the system Hamiltonian and from the time derivative of the density matrix

$$
\dot{E}(t)=\operatorname{Tr} \rho(t) \dot{H}(t)+\operatorname{Tr} \dot{\rho}(t) H(t),
$$

the latter being identified as heat flow which would be zero in a closed system evolving with unitary dynamics. We are interested in the first term, which measures the absorbed power from the external perturbation [142] and can be written as

$\dot{W}(t)=\operatorname{Tr} \rho(t) \dot{H}(t)=\sum_{i}\left(i \omega v_{i}^{*} e^{i \omega t}\left\langle b_{i}\right\rangle+\right.$ H.c. $)$.

Within linear response theory, the average of the bosonic field can be written as

$$
\left\langle b_{i}\right\rangle=\sum_{j} \int d t^{\prime} G_{i j}^{R}\left(t-t^{\prime}\right) v_{j}\left(t^{\prime}\right),
$$

where $G_{i j}^{R}\left(t-t^{\prime}\right)=-i \theta\left(t-t^{\prime}\right)\left\langle\left[b_{i}(t), b_{j}^{\dagger}\left(t^{\prime}\right)\right]\right\rangle$. Plugging this expression into Eq. (D4), we obtain the average absorbed power

$$
\dot{W}=\sum_{i j} v_{i}^{*} v_{j} \omega A_{i j}(\omega)
$$

written in terms of the spectral function $A_{i j}(\omega)=$ $(-1 / \pi) \operatorname{Im} G_{i j}^{R}(\omega)$. For a localized perturbation $v_{i}=v_{0} \delta_{i, 0}$, we obtain the result given in the main text, showing how an NDOS implies a negative absorption rate; namely, the system transfers some of the energy from the perturbation to the emitted radiation.

\section{APPENDIX E: HUBBARD-I IMPURITY SOLVER}

In this Appendix, we compute the impurity Green's functions in the simple Hubbard-I approximation discussed in Sec. IVA and show that the phase diagram computed in Hubbard-I coincides with the mean-field one. We restrict to study the symmetric phase, i.e., $\boldsymbol{\Phi}=0$, where equations involve one Nambu component and to the stationary regime where convolutions turn into product under Fourier transform.

Equation (24) is a closed second-order algebraic equation for $G^{\alpha \beta}(\omega)$, which is easily solved for the retarded and Keldysh components. The retarded Green's function is simply given by taking its retarded component, yielding

$$
J^{2} / z G^{R}(\omega)=\left[G_{0}^{R}(\omega)\right]^{-1}-\left[G^{R}(\omega)\right]^{-1},
$$

which can be solved and gives

$G^{R}(\omega)=\frac{z}{2 J^{2}} G_{0}^{R}(\omega)^{-1}\left(1-\sqrt{1-\frac{4 J^{2}}{z} G_{0}^{R}(\omega)^{2}}\right)$.

The inverse Keldysh Green's function is given by $\left[G^{-1}\right]^{K}=\left[G_{0}^{-1}\right]^{K}-\left(J^{2} / z\right) G^{K}$, that we invert with the standard relation $G^{K}=-G^{R}\left[G^{-1}\right]^{K} G^{A}$, giving

$$
G^{K}(\omega)=\frac{\left|G^{R}(\omega)\right|^{2} G_{0}^{K}(\omega)}{\left|G_{0}^{R}(\omega)\right|^{2}\left(1-\frac{J^{2}}{z}\left|G^{R}(\omega)\right|^{2}\right)} .
$$

We finally show that the Hubbard-I impurity solver gives the same phase boundary and critical frequency as Gutzwiller mean-field theory. In fact, if we plug the equation for the Hubbard-I retarded Green's function [Eq. (E1)] into the equation for the finite-frequency transition in DMFT [Eq. (40)] that we rewrite for convenience here

$$
\frac{1}{J_{c}}+G^{R}\left(\Omega_{c}, J_{c}\right)+\frac{J_{c}}{z}\left[G^{R}\left(\Omega_{c}, J_{c}\right)\right]^{2}=0,
$$

we obtain

$$
G^{R}\left(\Omega_{c}, J_{c}\right)\left(J_{c}+\left[G_{0}^{R}\left(\Omega_{c}\right)\right]^{-1}\right)=0,
$$

where the term in parentheses is exactly the Gutzwiller mean-field condition for critical hopping and frequency [see Eq. (44) or (40) for $z=\infty$ ]. We conclude, therefore, that the reduction of the ordered phase discussed in the main text and the dependence of $\Omega_{c}$ from the hopping are key features of the NCA impurity solver.

\section{APPENDIX F: STATIONARY STATE IN DMFT NCA}

A complete steady-state DMFT NCA procedure requires one to compute the steady-state density matrix of the impurity, which is defined by $\rho_{s}=\hat{\mathcal{V}}(\infty, 0) \rho_{0}$, where we assume a unique steady state. While, in principle, this computation would require one to perform the full transient dynamics from an arbitrary initial condition, here we show how to obtain $\rho_{s}$ directly from the stationary-state propagator $\hat{\mathcal{V}}\left(t-t^{\prime}\right)$. The steady-state density matrix satisfies the condition $\lim _{t \rightarrow \infty} \partial_{t} \rho(t)=\lim _{t \rightarrow \infty} \partial_{t} \hat{\mathcal{V}}(t, 0) \rho_{0}=0$, where $\rho_{0}$ is an arbitrary initial state. Using the Dyson equation (26), this condition translates into the equation

$$
\lim _{t \rightarrow \infty}\left[\hat{\mathcal{L}}_{\text {eff }}(t) \hat{\mathcal{V}}(t, 0)+\int_{0}^{t} d \tau \hat{S}(t, t-\tau) \hat{\mathcal{V}}(t-\tau, 0)\right] \rho_{0}=0
$$


where we perform a change of variable in the convolution integral. The NCA self-energy $\hat{S}(t, t-\tau)$ in the equation above depends on the hybridization function of the bath $\boldsymbol{\Delta}$ [see Eq. (27)], which is itself related to the local Green's function of the system by the DMFT self-consistency. Therefore, the assumption that for $t \rightarrow \infty$ the system reaches a unique steady state, forgetting about its initial condition, translates within DMFT to a requirement on the behavior of the NCA self-energy for large values of its argument. In particular, $\hat{S}(t, t-\tau)$ must vanish for $\tau \sim t \rightarrow \infty$, restricting the time convolution in the Dyson equation to times of the order $\tau \sim \tau_{*} \ll t$. We can, therefore, safely take the long time limit $t \rightarrow \infty$ in Eq. (F1), use the fact that in this limit the self-energy becomes translational invariant, $\hat{S}(t, t-\tau) \rightarrow \hat{S}(\tau)$, and the state $\hat{\mathcal{V}}(t-\tau, 0) \rho_{0}$ approaches the stationary state $\rho_{s}$, and finally send the scale $\tau_{*}$ to infinity, yielding the eigenvalue condition

$$
\left(\hat{\mathcal{L}}_{\text {eff }}(\infty)+\int_{0}^{\infty} d \tau \hat{S}(\tau)\right) \rho_{s}=0
$$

This condition depends only on the stationary-state propagator $\hat{\mathcal{V}}(\tau)$ through the NCA self-energy $\hat{S}(\tau)$, allowing us to compute the stationary density matrix $\rho_{s}$ in the steadystate DMFT procedure.

\section{APPENDIX G: NCA GREEN FUNCTIONS DERIVATION}

In this Appendix, we show how to compute impurity Green functions knowing $\mathcal{V}\left(t, t^{\prime}\right)$. From the impurity action Eq. (9), the trace of the density matrix at infinite time is obtained by

$$
Z=\operatorname{tr} \rho(\infty)=\int \mathcal{D}\left[\bar{b}_{\alpha} b_{\alpha}\right] e^{i S_{\mathrm{loc}}\left[\bar{b}_{\alpha, a}, b_{\beta, b}\right]+i \int_{0}^{\infty} d t \sum_{a} \alpha \bar{\Phi}_{\mathrm{eff} \alpha a}(t) b_{\alpha a}(t)-(i / 2) \int_{0}^{\infty} \int_{0}^{\infty} d t_{1} d t_{2} \sum_{a b} \bar{b}_{\alpha a}\left(t_{1}\right) \alpha \beta \Delta_{a b}^{\alpha \beta}\left(t_{1}, t_{2}\right) b_{\beta b}\left(t_{2}\right)},
$$

where we make explicit both Keldysh $\alpha, \beta \in\{+,-\}$ and Nambu $a, b$ indices, as in Sec. IV B. One-particle Green functions can be obtained as functional derivatives of $Z$ :

$$
G_{a b}^{\alpha \beta}\left(t, t^{\prime}\right)-i \phi_{\alpha a}(t) \phi_{\beta b}^{\dagger}\left(t^{\prime}\right)=-i\left\langle b_{\alpha a}(t) \bar{b}_{\beta b}\left(t^{\prime}\right)\right\rangle=2 \alpha \beta \frac{\delta Z}{\delta \Delta_{b a}^{\beta \alpha}\left(t^{\prime}, t\right)}
$$

We remark that the derivative over $\Delta$ gives both the connected components $G_{a b}^{\alpha \beta}$, as defined in the main text Eq. (14), and the disconnected ones $-i \phi_{\alpha a}(t) \phi_{\beta b}^{\dagger}\left(t^{\prime}\right)$. The density matrix trace can equally be expressed in terms of the evolution superoperator: $Z=\operatorname{tr}[\rho(\infty)]=\operatorname{tr}[\hat{\mathcal{V}}(\infty, 0) \rho(0)]$. Using the Dyson equation $(26)$ for $\hat{\mathcal{V}}(\infty, 0)$, we get

$-i\left\langle b_{\alpha a}(t) \bar{b}_{\beta b}\left(t^{\prime}\right)\right\rangle=2 \alpha \beta \operatorname{tr}\left\{\int_{0}^{\infty} d t_{1} \int_{0}^{t_{1}} d t_{2}\left[\hat{\mathcal{V}}_{0}\left(\infty, t_{1}\right) \frac{\delta \hat{S}\left(t_{1}, t_{2}\right)}{\delta \Delta_{b a}^{\beta \alpha}\left(t^{\prime}, t\right)} \hat{\mathcal{V}}\left(t_{2}, 0\right)+\hat{\mathcal{V}}_{0}\left(\infty, t_{1}\right) \hat{S}\left(t_{1}, t_{2}\right) \frac{\delta \hat{\mathcal{V}}\left(t_{2}, 0\right)}{\delta \Delta_{b a}^{\beta \alpha}\left(t^{\prime}, t\right)}\right] \rho(0)\right\}$

In the last expression, we first drop all $\hat{\mathcal{V}}_{0}$ 's, as they leave the trace unchanged. The second contribution to the trace vanishes, because $\operatorname{tr}\left(\hat{S}\left(t_{1}, t_{2}\right) \bullet\right)=0$ for $t_{1}>t_{2}$, as dictated by the time integrals, as we now show. In order to do this integration, we report here the NCA self-energy Eq. (27) for simplicity:

$$
\hat{S}\left(t_{1}, t_{2}\right)=-\frac{i}{2} \sum_{\substack{\alpha \beta \\ a b}} \alpha \beta\left[\Delta_{b a}^{\beta \alpha}\left(t_{1}, t_{2}\right) \hat{b}_{\beta b}^{\dagger} \hat{\mathcal{V}}\left(t_{1}, t_{2}\right) \hat{b}_{\alpha a}+\Delta_{a b}^{\alpha \beta}\left(t_{2}, t_{1}\right) \hat{b}_{\beta b} \hat{\mathcal{V}}\left(t_{1}, t_{2}\right) \hat{b}_{\alpha a}^{\dagger}\right]
$$

We remark that, for the cyclic property of the trace, it holds that $\operatorname{tr}\left[\hat{X}_{+} \bullet\right]=\operatorname{tr}\left[\hat{X}_{-} \bullet\right], \hat{X}_{\beta}$ being a generic superoperator. Then, we can fix the $\hat{b}_{\beta}^{\dagger}, \hat{b}_{\beta}$ superoperators to be $\hat{b}_{+}^{\dagger}, \hat{b}_{+}$under the trace, getting

$$
\begin{aligned}
\operatorname{tr}\left[\hat{S}\left(t_{1}, t_{2}\right) \bullet\right] & =\sum_{a, b} \sum_{\alpha \beta \in\{+,-\}}-\alpha \beta \frac{i}{2} \operatorname{tr}\left[\Delta_{b a}^{\beta \alpha}\left(t_{1}, t_{2}\right) \hat{b}_{\beta b}^{\dagger} \hat{\mathcal{V}}\left(t_{1}, t_{2}\right) \hat{b}_{\alpha a} \bullet+\Delta_{a b}^{\alpha \beta}\left(t_{2}, t_{1}\right) \hat{b}_{\beta b} \hat{\mathcal{V}}\left(t_{1}, t_{2}\right) \hat{b}_{\alpha a}^{\dagger} \bullet \cdot\right] \\
& =\sum_{a, b} \sum_{\alpha \in\{+,-\}}-\alpha \frac{i}{2} \operatorname{tr}\left\{\sum_{\beta \in\{+,-\}}\left[\beta \Delta_{b a}^{\beta \alpha}\left(t_{1}, t_{2}\right)\right] \hat{b}_{+b}^{\dagger} \hat{\mathcal{V}}\left(t_{1}, t_{2}\right) \hat{b}_{\alpha a} \bullet+\sum_{\beta \in\{+,-\}}\left[\beta \Delta_{a b}^{\alpha \beta}\left(t_{2}, t_{1}\right)\right] \hat{b}_{+b} \hat{\mathcal{V}}\left(t_{1}, t_{2}\right) \hat{b}_{\alpha a}^{\dagger} \bullet\right\} .
\end{aligned}
$$


Finally, we remark that the two sums over $\beta$ in the last line vanish, respectively, because of the identities on the Keldysh components $\Delta_{b a}^{++}\left(t_{1}, t_{2}\right)=\Delta_{b a}^{-+}\left(t_{1}, t_{2}\right), \Delta_{b a}^{+-}\left(t_{1}, t_{2}\right)=\Delta_{b a}^{--}\left(t_{1}, t_{2}\right)$ and $\Delta_{a b}^{++}\left(t_{2}, t_{1}\right)=\Delta_{a b}^{+-}\left(t_{2}, t_{1}\right), \Delta_{a b}^{-+}\left(t_{2}, t_{1}\right)=$ $\Delta_{a b}^{--}\left(t_{2}, t_{1}\right)$, which hold for $t_{1}>t_{2}$ according to the definition of $\Delta$ in terms of bath Green functions: Schematically, its Keldysh components are given by $\Delta^{\beta \alpha}\left(t_{1}, t_{2}\right) \sim \sum \ldots\left\langle T_{C} c\left(t_{1}, \beta\right) c^{\dagger}\left(t_{2}, \alpha\right)\right\rangle$ with $c$ and $c^{\dagger}$ bath operators and $T_{C}$ the contourtime-ordering operator. Going back to Eq. (G3), from its first term we get

$$
G_{a b}^{\alpha \beta}\left(t, t^{\prime}\right)-i \phi_{\alpha a}(t) \phi_{\beta b}^{\dagger}\left(t^{\prime}\right)=2 \alpha \beta \operatorname{tr}\left\{\int_{0}^{\infty} d t_{1} \int_{0}^{t_{1}} d t_{2} \frac{\delta \hat{S}\left(t_{1}, t_{2}\right)}{\delta \Delta_{b a}^{\beta \alpha}\left(t^{\prime}, t\right)} \hat{\mathcal{V}}\left(t_{2}, 0\right) \rho(0)\right\} .
$$

Taking derivatives of the NCA self-energy, we get two contributions of the form $\simeq \operatorname{tr}\left[(\delta \Delta / \delta \Delta) X_{1} \mathcal{V} X_{2}+\Delta X_{1}(\delta \mathcal{V} / \delta \Delta) X_{2}\right]$. The second one vanishes, and the proof is analogous to Eq. (G5), where we show that the trace of the NCA self-energy vanishes. For the first term, we need to remember that $t_{1}>t_{2}$ and, thus, that we have to distinguish the two cases $t>t^{\prime}$ and $t<t^{\prime}$, getting

$$
\frac{\delta \hat{S}\left(t_{1}, t_{2}\right)}{\delta \Delta_{b a}^{\beta \alpha}\left(t^{\prime}, t\right)}=-\frac{i}{2} \alpha \beta\left[\hat{b}_{\alpha a} \hat{\mathcal{V}}\left(t, t^{\prime}\right) \hat{b}_{\beta b}^{\dagger} \theta\left(t-t^{\prime}\right) \delta\left(t_{1}-t\right) \delta\left(t_{2}-t^{\prime}\right)+\hat{b}_{\beta b}^{\dagger} \hat{\mathcal{V}}\left(t^{\prime}, t\right) \hat{b}_{\alpha a} \theta\left(t^{\prime}-t\right) \delta\left(t_{1}-t^{\prime}\right) \delta\left(t_{2}-t\right)\right],
$$

and the Green functions are eventually given by

$$
G_{a b}^{\alpha \beta}\left(t, t^{\prime}\right)-i \phi_{\alpha a}(t) \phi_{\beta b}^{\dagger}\left(t^{\prime}\right)=-i\left\{\operatorname{tr}\left[\hat{b}_{\alpha a} \hat{\mathcal{V}}\left(t, t^{\prime}\right) \hat{b}_{\beta b}^{\dagger} \hat{\mathcal{V}}\left(t^{\prime}, 0\right) \rho(0)\right] \theta\left(t-t^{\prime}\right)+\operatorname{tr}\left[\hat{b}_{\beta b}^{\dagger} \hat{\mathcal{V}}\left(t^{\prime}, t\right) \hat{b}_{\alpha a} \hat{\mathcal{V}}(t, 0) \rho(0)\right] \theta\left(t^{\prime}-t\right)\right\}
$$

that is equivalent to Eq. (30) of the main text. This equation has the same form of the quantum regression formulas for one-particle Green functions for Markovian systems and shows that these generalize to the case of non-Markovian baths within the NCA approximation.

[1] J. I. Cirac and P. Zoller, Quantum Computations with Cold Trapped Ions, Phys. Rev. Lett. 74, 4091 (1995).

[2] J. M. Raimond, M. Brune, and S. Haroche, Manipulating Quantum Entanglement with Atoms and Photons in a Cavity, Rev. Mod. Phys. 73, 565 (2001).

[3] R. J. Schoelkopf and S. M. Girvin, Wiring up Quantum Systems, Nature (London) 451, 664 (2008).

[4] M. Aspelmeyer, T. J. Kippenberg, and F. Marquardt, Cavity Optomechanics, Rev. Mod. Phys. 86, 1391 (2014).

[5] I. Bloch, J. Dalibard, and S. Nascimbène, Quantum Simulations with Ultracold Quantum Gases, Nat. Phys. 8, 267 (2012).

[6] A. Browaeys and T. Lahaye, Many-Body Physics with Individually Controlled Rydberg Atoms, Nat. Phys. 16, 132 (2020).

[7] R. Blatt and C.F. Roos, Quantum Simulations with Trapped Ions, Nat. Phys. 8, 277 (2012).

[8] A. A. Houck, H. E. Türeci, and J. Koch, On-Chip Quantum Simulation with Superconducting Circuits, Nat. Phys. 8, 292 (2012).

[9] Y. Salathé, M. Mondal, M. Oppliger, J. Heinsoo, P. Kurpiers, A. Potočnik, A. Mezzacapo, U. Las Heras, L. Lamata, E. Solano, S. Filipp, and A. Wallraff, Digital Quantum Simulation of Spin Models with Circuit Quantum Electrodynamics, Phys. Rev. X 5, 021027 (2015).
[10] J. P. Martínez, S. Léger, N. Gheeraert, R. Dassonneville, L. Planat, F. Foroughi, Y. Krupko, O. Buisson, C. Naud, W. Hasch-Guichard, S. Florens, I. Snyman, and N. Roch, A Tunable Josephson Platform to Explore Many-Body Quantum Optics in Circuit-QED, npj Quantum Inf. 5, 19 (2019).

[11] I. Carusotto, A. A. Houck, A. J. Kollár, P. Roushan, D. I. Schuster, and J. Simon, Photonic Materials in Circuit Quantum Electrodynamics, Nat. Phys. 16, 268 (2020).

[12] J. M. Fink, A. Dombi, A. Vukics, A. Wallraff, and P. Domokos, Observation of the Photon-Blockade Breakdown Phase Transition, Phys. Rev. X 7, 011012 (2017).

[13] M. Fitzpatrick, N. M. Sundaresan, A. C. Y. Y. Li, J. Koch, and A. A. Houck, Observation of a Dissipative Phase Transition in a One-Dimensional Circuit QED Lattice, Phys. Rev. X 7, 011016 (2017).

[14] T. Fink, A. Schade, S. Höfling, C. Schneider, and A. Imamoglu, Signatures of a Dissipative Phase Transition in Photon Correlation Measurements, Nat. Phys. 14, 365 (2018).

[15] J. Raftery, D. Sadri, S. Schmidt, H. E. Türeci, and A. A. Houck, Observation of a Dissipation-Induced Classical to Quantum Transition, Phys. Rev. X 4, 031043 (2014).

[16] R. Ma, B. Saxberg, C. Owens, N. Leung, Y. Lu, J. Simon, and D. I. Schuster, A Dissipatively Stabilized Mott Insulator of Photons, Nature (London) 566, 51 (2019).

[17] N. Syassen, D. M. Bauer, M. Lettner, T. Volz, D. Dietze, J. J. García-Ripoll, J. I. Cirac, G. Rempe, and S. Dürr, Strong Dissipation Inhibits Losses and Induces Correlations in Cold Molecular Gases, Science 320, 1329 (2008).

[18] T. Tomita, S. Nakajima, I. Danshita, Y. Takasu, and Y. Takahashi, Observation of the Mott Insulator to Superfluid Crossover of a Driven-Dissipative Bose-Hubbard System, Sci. Adv. 3, e1701513 (2017). 
[19] H. P. Lüschen, P. Bordia, S. S. Hodgman, M. Schreiber, S. Sarkar, A. J. Daley, M. H. Fischer, E. Altman, I. Bloch, and U. Schneider, Signatures of Many-Body Localization in a Controlled Open Quantum System, Phys. Rev. X 7, 011034 (2017).

[20] R. Bouganne, M. B. Aguilera, A. Ghermaoui, J. Beugnon, and F. Gerbier, Anomalous Decay of Coherence in a Dissipative Many-Body System, Nat. Phys. 16, 21 (2020).

[21] I. Carusotto and C. Ciuti, Quantum Fluids of Light, Rev. Mod. Phys. 85, 299 (2013).

[22] H. Ritsch, P. Domokos, F. Brennecke, and T. Esslinger, Cold Atoms in Cavity-Generated Dynamical Optical Potentials, Rev. Mod. Phys. 85, 553 (2013).

[23] S. Schmidt and J. Koch, Circuit QED Lattices: Towards Quantum Simulation with Superconducting Circuits, Ann. Phys. (Berlin) 525, 395 (2013).

[24] K. Le Hur, L. Henriet, A. Petrescu, K. Plekhanov, G. Roux, and M. Schiró, Many-Body Quantum Electrodynamics Networks: Non-equilibrium Condensed Matter Physics with Light, C.R. Phys. 17, 808 (2016).

[25] C. Noh and D. G. Angelakis, Quantum Simulations and Many-Body Physics with Light, Rep. Prog. Phys. 80, 016401 (2017).

[26] M. J. Hartmann, Quantum Simulation with Interacting Photons, J. Opt. 18, 104005 (2016).

[27] H. P. Breuer and F. Petruccione, The Theory of Open Quantum Systems, 1st ed. (Oxford University, New York, 2007), Vol. 9780199213.

[28] S. Diehl, A. Micheli, A. Kantian, B. Kraus, H. P. Büchler, and P. Zoller, Quantum States and Phases in Driven Open Quantum Systems with Cold Atoms, Nat. Phys. 4, 878 (2008).

[29] F. Verstraete, M. M. Wolf, and J. I. Cirac, Quantum Computation and Quantum-State Engineering Driven by Dissipation, Nat. Phys. 5, 633 (2009).

[30] K. W. Murch, U. Vool, D. Zhou, S. J. Weber, S. M. Girvin, and I. Siddiqi, Cavity-Assisted Quantum Bath Engineering, Phys. Rev. Lett. 109, 183602 (2012).

[31] Z. Leghtas, S. Touzard, I. M. Pop, A. Kou, B. Vlastakis, A. Petrenko, K. M. Sliwa, A. Narla, S. Shankar, M. J. Hatridge, M. Reagor, L. Frunzio, R. J. Schoelkopf, M. Mirrahimi, and M. H. Devoret, Confining the State of Light to a Quantum Manifold by Engineered Two-Photon Loss, Science 347, 853 (2015).

[32] S. Diehl, A. Tomadin, A. Micheli, R. Fazio, and P. Zoller, Dynamical Phase Transitions and Instabilities in Open Atomic Many-Body Systems, Phys. Rev. Lett. 105, 015702 (2010).

[33] D. Poletti, J.-S. Bernier, A. Georges, and C. Kollath, Interaction-Induced Impeding of Decoherence and Anomalous Diffusion, Phys. Rev. Lett. 109, 045302 (2012).

[34] D. Poletti, P. Barmettler, A. Georges, and C. Kollath, Emergence of Glasslike Dynamics for Dissipative and Strongly Interacting Bosons, Phys. Rev. Lett. 111, 195301 (2013).

[35] M. Ludwig and F. Marquardt, Quantum Many-Body Dynamics in Optomechanical Arrays, Phys. Rev. Lett. 111, 073603 (2013).
[36] L. M. Sieberer, S. D. Huber, E. Altman, and S. Diehl, Dynamical Critical Phenomena in Driven-Dissipative Systems, Phys. Rev. Lett. 110, 195301 (2013).

[37] J. Marino and S. Diehl, Driven Markovian Quantum Criticality, Phys. Rev. Lett. 116, 070407 (2016).

[38] M. Schiró, C. Joshi, M. Bordyuh, R. Fazio, J. Keeling, and H. E. Türeci, Exotic Attractors of the Nonequilibrium Rabi-Hubbard Model, Phys. Rev. Lett. 116, 143603 (2016).

[39] F. Minganti, A. Biella, N. Bartolo, and C. Ciuti, Spectral Theory of Liouvillians for Dissipative Phase Transitions, Phys. Rev. A 98, 042118 (2018).

[40] R. Rota, F. Minganti, C. Ciuti, and V. Savona, Quantum Critical Regime in a Quadratically Driven Nonlinear Photonic Lattice, Phys. Rev. Lett. 122, 110405 (2019).

[41] J. T. Young, A. V. Gorshkov, M. Foss-Feig, and M. F. Maghrebi, Nonequilibrium Fixed Points of Coupled Ising Models, Phys. Rev. X 10, 011039 (2020).

[42] A. J. Daley, Quantum Trajectories and Open Many-Body Quantum Systems, Adv. Phys. 63, 77 (2014).

[43] F. Verstraete, J. J. García-Ripoll, and J. I. Cirac, Matrix Product Density Operators: Simulation of FiniteTemperature and Dissipative Systems, Phys. Rev. Lett. 93, 207204 (2004).

[44] M. Zwolak and G. Vidal, Mixed-State Dynamics in OneDimensional Quantum Lattice Systems: A TimeDependent Superoperator Renormalization Algorithm, Phys. Rev. Lett. 93, 207205 (2004).

[45] D. Kilda and J. Keeling, Fluorescence Spectrum and Thermalization in a Driven Coupled Cavity Array, Phys. Rev. Lett. 122, 043602 (2019).

[46] A. Kshetrimayum, H. Weimer, and R. Orús, A Simple Tensor Network Algorithm for Two-Dimensional Steady States, Nat. Commun. 8, 1291 (2017).

[47] H. Landa, M. Schiró, and G. Misguich, Multistability of Driven-Dissipative Quantum Spins, Phys. Rev. Lett. 124, 043601 (2020).

[48] D. Kilda, A. Biella, M. Schiró, R. Fazio, and J. Keeling, On the Stability of the Infinite Projected Entangled Pair Operator Ansatz for Driven-Dissipative 2D Lattices, arXiv:2012.03095.

[49] S. Finazzi, A. Le Boité, F. Storme, A. Baksic, and C. Ciuti, Corner-Space Renormalization Method for DrivenDissipative Two-Dimensional Correlated Systems, Phys. Rev. Lett. 115, 080604 (2015).

[50] H. Weimer, Variational Principle for Steady States of Dissipative Quantum Many-Body Systems, Phys. Rev. Lett. 114, 040402 (2015).

[51] J. Jin, A. Biella, O. Viyuela, L. Mazza, J. Keeling, R. Fazio, and D. Rossini, Cluster Mean-Field Approach to the Steady-State Phase Diagram of Dissipative Spin Systems, Phys. Rev. X 6, 031011 (2016).

[52] L. M. Sieberer, M. Buchhold, and S. Diehl, Keldysh Field Theory for Driven Open Quantum Systems, Rep. Prog. Phys. 79, 096001 (2016).

[53] M. Biondi, S. Lienhard, G. Blatter, H. E. Türeci, and S. Schmidt, Spatial Correlations in Driven-Dissipative Photonic Lattices, New J. Phys. 19, 125016 (2017). 
[54] F. Vicentini, F. Minganti, R. Rota, G. Orso, and C. Ciuti, Critical Slowing Down in Driven-Dissipative BoseHubbard Lattices, Phys. Rev. A 97, 013853 (2018).

[55] H. Weimer, A. Kshetrimayum, and R. Orús, Simulation Methods for Open Quantum Many-Body Systems, Rev. Mod. Phys. 93, 015008 (2021).

[56] F. Vicentini, A. Biella, N. Regnault, and C. Ciuti, Variational Neural-Network Ansatz for Steady States in Open Quantum Systems, Phys. Rev. Lett. 122, 250503 (2019).

[57] N. Yoshioka and R. Hamazaki, Constructing Neural Stationary States for Open Quantum Many-Body Systems, Phys. Rev. B 99, 214306 (2019).

[58] A. Nagy and V. Savona, Variational Quantum Monte Carlo Method with a Neural-Network Ansatz for Open Quantum Systems, Phys. Rev. Lett. 122, 250501 (2019).

[59] M. J. Hartmann and G. Carleo, Neural-Network Approach to Dissipative Quantum Many-Body Dynamics, Phys. Rev. Lett. 122, 250502 (2019).

[60] W. Metzner and D. Vollhardt, Correlated Lattice Fermions in Infinite Dimensions, Phys. Rev. Lett. 62, 324 (1989).

[61] A. Georges and G. Kotliar, Hubbard Model in Infinite Dimensions, Phys. Rev. B 45, 6479 (1992).

[62] A. Georges, G. Kotliar, W. Krauth, and M. J. Rozenberg, Dynamical Mean-Field Theory of Strongly Correlated Fermion Systems and the Limit of Infinite Dimensions, Rev. Mod. Phys. 68, 13 (1996).

[63] K. Byczuk and D. Vollhardt, Correlated Bosons on a Lattice: Dynamical Mean-Field Theory for Bose-Einstein Condensed and Normal Phases, Phys. Rev. B 77, 235106 (2008).

[64] P. Anders, E. Gull, L. Pollet, M. Troyer, and P. Werner, Dynamical Mean Field Solution of the Bose-Hubbard Model, Phys. Rev. Lett. 105, 096402 (2010).

[65] P. Anders, E. Gull, L. Pollet, M. Troyer, and P. Werner, Dynamical Mean-Field Theory for Bosons, New J. Phys. 13, 075013 (2011).

[66] H. Aoki, N. Tsuji, M. Eckstein, M. Kollar, T. Oka, and P. Werner, Nonequilibrium Dynamical Mean-Field Theory and Its Applications, Rev. Mod. Phys. 86, 779 (2014).

[67] J. Panas, M. Pasek, A. Dhar, T. Qin, A. Geißler, M. HafezTorbati, M. E. Sorantin, I. Titvinidze, and W. Hofstetter, Density-Wave Steady-State Phase of Dissipative Ultracold Fermions with Nearest-Neighbor Interactions, Phys. Rev. B 99, 115125 (2019).

[68] H. U. R. Strand, M. Eckstein, and P. Werner, Nonequilibrium Dynamical Mean-Field Theory for Bosonic Lattice Models, Phys. Rev. X 5, 011038 (2015).

[69] M. Schiro and O. Scarlatella, Quantum Impurity Models Coupled to Markovian and Non-Markovian Baths, J. Chem. Phys. 151, 044102 (2019).

[70] T. E. Lee and H. R. Sadeghpour, Quantum Synchronization of Quantum Van der Pol Oscillators with Trapped Ions, Phys. Rev. Lett. 111, 234101 (2013).

[71] N. Lörch, E. Amitai, A. Nunnenkamp, and C. Bruder, Genuine Quantum Signatures in Synchronization of Anharmonic Self-Oscillators, Phys. Rev. Lett. 117, 073601 (2016).

[72] S. Walter, A. Nunnenkamp, and C. Bruder, Quantum Synchronization of a Driven Self-Sustained Oscillator, Phys. Rev. Lett. 112, 094102 (2014).
[73] S. Walter, A. Nunnenkamp, and C. Bruder, Quantum Synchronization of Two Van der Pol oscillators, Ann. Phys. (Berlin) 527, 131 (2015).

[74] A. Roulet and C. Bruder, Synchronizing the Smallest Possible System, Phys. Rev. Lett. 121, 053601 (2018).

[75] A. Roulet and C. Bruder, Quantum Synchronization and Entanglement Generation, Phys. Rev. Lett. 121, 063601 (2018).

[76] S. Dutta and N. R. Cooper, Critical Response of a Quantum Van der Pol Oscillator, Phys. Rev. Lett. 123, 250401 (2019).

[77] G. L. Giorgi, F. Galve, G. Manzano, P. Colet, and R. Zambrini, Quantum Correlations and Mutual Synchronization, Phys. Rev. A 85, 052101 (2012).

[78] G. Manzano, F. Galve, G. L. Giorgi, E. Hernández-García, and R. Zambrini, Synchronization, Quantum Correlations and Entanglement in Oscillator Networks, Sci. Rep. 3, 1439 (2013).

[79] G.-J. Qiao, H.-X. Gao, H.-D. Liu, and X. X. Yi, Quantum Synchronization of Two Mechanical Oscillators in Coupled Optomechanical Systems with Kerr Nonlinearity, Sci. Rep. 8, 15614 (2018).

[80] S. Sonar, M. Hajdušek, M. Mukherjee, R. Fazio, V. Vedral, S. Vinjanampathy, and L.-C. Kwek, Squeezing Enhances Quantum Synchronization, Phys. Rev. Lett. 120, 163601 (2018).

[81] N. Jaseem, M. Hajdušek, V. Vedral, R. Fazio, L.-C. Kwek, and S. Vinjanampathy, Quantum Synchronization in Nanoscale Heat Engines, Phys. Rev. E 101, 020201(R) (2020).

[82] J. Tindall, C. S. Muñoz, B. Buča, and D. Jaksch, Quantum Synchronisation Enabled by Dynamical Symmetries and Dissipation, New J. Phys. 22, 013026 (2020).

[83] M. P. A. Fisher, P. B. Weichman, G. Grinstein, and D. S. Fisher, Boson Localization and the Superfluid-Insulator Transition, Phys. Rev. B 40, 546 (1989).

[84] A. V. Joura, J. K. Freericks, and T. Pruschke, Steady-State Nonequilibrium Density of States of Driven Strongly Correlated Lattice Models in Infinite Dimensions, Phys. Rev. Lett. 101, 196401 (2008).

[85] J. Li, C. Aron, G. Kotliar, and J. E. Han, Electric-FieldDriven Resistive Switching in the Dissipative Hubbard Model, Phys. Rev. Lett. 114, 226403 (2015).

[86] E. Arrigoni, M. Knap, and W. von der Linden, Nonequilibrium Dynamical Mean-Field Theory: An Auxiliary Quantum Master Equation Approach, Phys. Rev. Lett. 110, 086403 (2013).

[87] I. Titvinidze, M. E. Sorantin, A. Dorda, W. von der Linden, and E. Arrigoni, Charge Redistribution in Correlated Heterostuctures within Nonequilibrium Real-Space Dynamical Mean-Field Theory, Phys. Rev. B 98, 035146 (2018).

[88] A. Matthies, J. Li, and M. Eckstein, Control of Competing Superconductivity and Charge Order by Nonequilibrium Currents, Phys. Rev. B 98, 180502(R) (2018).

[89] Y. Murakami and P. Werner, Nonequilibrium Steady States of Electric Field Driven Mott Insulators, Phys. Rev. B 98, 075102 (2018).

[90] N. Tsuji, T. Oka, and H. Aoki, Correlated Electron Systems Periodically Driven out of Equilibrium: Floquet + DMFT Formalism, Phys. Rev. B 78, 235124 (2008). 
[91] Y. Murakami, N. Tsuji, M. Eckstein, and P. Werner, Nonequilibrium Steady States and Transient Dynamics of Conventional Superconductors under Phonon Driving, Phys. Rev. B 96, 045125 (2017).

[92] T. Qin and W. Hofstetter, Spectral Functions of a TimePeriodically Driven Falicov-Kimball Model: Real-Space Floquet Dynamical Mean-Field Theory Study, Phys. Rev. B 96, 075134 (2017).

[93] J. Li and M. Eckstein, Nonequilibrium Steady-State Theory of Photodoped Mott Insulators, Phys. Rev. B 103, 045133 (2021).

[94] M. Eckstein and P. Werner, Photoinduced States in a Mott Insulator, Phys. Rev. Lett. 110, 126401 (2013).

[95] D. Golež, M. Eckstein, and P. Werner, Dynamics of Screening in Photodoped Mott Insulators, Phys. Rev. B 92, 195123 (2015).

[96] H.-T. T. Chen, G. Cohen, A. J. Millis, and D. R. Reichman, Anderson-Holstein Model in Two Flavors of the Noncrossing Approximation, Phys. Rev. B 93, 174309 (2016).

[97] N. Bittner, D. Golež, H. U. R. Strand, M. Eckstein, and P. Werner, Coupled Charge and Spin Dynamics in a Photoexcited Doped Mott Insulator, Phys. Rev. B 97, 235125 (2018).

[98] F. Peronaci, O. Parcollet, and M. Schiró, Enhancement of Local Pairing Correlations in Periodically Driven Mott Insulators, Phys. Rev. B 101, 161101(R) (2020).

[99] R. Rota, F. Minganti, C. Ciuti, and V. Savona, Quantum Critical Regime in a Quadratically Driven Nonlinear Photonic Lattice, Phys. Rev. Lett. 122, 110405 (2019).

[100] R. Lescanne, M. Villiers, T. Peronnin, A. Sarlette, M. Delbecq, B. Huard, T. Kontos, M. Mirrahimi, and Z. Leghtas, Exponential Suppression of Bit-Flips in a Qubit Encoded in an Oscillator, Nat. Phys. 16, 509 (2020).

[101] M. J. Hartmann, F. G. S. L. Brandão, and M. B. Plenio, Strongly Interacting Polaritons in Coupled Arrays of Cavities, Nat. Phys. 2, 849 (2006).

[102] D. G. Angelakis, M. F. Santos, and S. Bose, PhotonBlockade-Induced Mott Transitions and xy Spin Models in Coupled Cavity Arrays, Phys. Rev. A 76, 031805(R) (2007).

[103] M. J. Hartmann, Polariton Crystallization in Driven Arrays of Lossy Nonlinear Resonators, Phys. Rev. Lett. 104, 113601 (2010).

[104] J. Jin, D. Rossini, R. Fazio, M. Leib, and M. J. Hartmann, Photon Solid Phases in Driven Arrays of Nonlinearly Coupled Cavities, Phys. Rev. Lett. 110, 163605 (2013).

[105] A. Le Boité, G. Orso, and C. Ciuti, Steady-State Phases and Tunneling-Induced Instabilities in the Driven Dissipative Bose-Hubbard Model, Phys. Rev. Lett. 110, 233601 (2013).

[106] J. Lebreuilly, A. Biella, F. Storme, D. Rossini, R. Fazio, C. Ciuti, and I. Carusotto, Stabilizing Strongly Correlated Photon Fluids with Non-Markovian Reservoirs, Phys. Rev. A 96, 033828 (2017).

[107] M. Foss-Feig, P. Niroula, J. T. Young, M. Hafezi, A. V. Gorshkov, R. M. Wilson, and M. F. Maghrebi, Emergent Equilibrium in Many-Body Optical Bistability, Phys. Rev. A 95, 043826 (2017).
[108] M. Biondi, G. Blatter, H. E. Türeci, and S. Schmidt, Nonequilibrium Gas-Liquid Transition in the DrivenDissipative Photonic Lattice, Phys. Rev. A 96, 043809 (2017).

[109] O. Scarlatella, R. Fazio, and M. Schiró, Emergent Finite Frequency Criticality of Driven-Dissipative Correlated Lattice Bosons, Phys. Rev. B 99, 064511 (2019).

[110] V. V. Dodonov and S. S. Mizrahi, Exact Stationary Photon Distributions due to Competition between One- and TwoPhoton Absorption and Emission, J. Phys. A 30, 5657 (1997).

[111] M. Dykman, Heating and Cooling of Local and Quasilocal Vibrations by a Nonresonance Field, Sov. Phys. Solid State 20, 1306 (1978).

[112] We are not limited to the coordination numbers we span in Fig. 2, and we can run DMFT equations for smaller values of $z$, but computing the phase diagram becomes particularly challenging, as it moves toward higher pump-to-loss $r$ values, as shown in Fig. 2, which are numerically hard to access, requiring bigger Hilbert space sizes.

[113] O. Scarlatella, A. A. Clerk, and M. Schirò, Spectral Functions and Negative Density of States of a DrivenDissipative Nonlinear Quantum Resonator, New J. Phys. 21, 043040 (2018).

[114] B. Buča, J. Tindall, and D. Jaksch, Non-stationary Coherent Quantum Many-Body Dynamics through Dissipation, Nat. Commun. 10, 1730 (2019).

[115] F. Iemini, A. Russomanno, J. Keeling, M. Schirò, M. Dalmonte, and R. Fazio, Boundary Time Crystals, Phys. Rev. Lett. 121, 035301 (2018).

[116] S. H. Strogatz and R. E. Mirollo, Stability of Incoherence in a Population of Coupled Oscillators, J. Stat. Phys. 63, 613 (1991).

[117] P. C. Matthews, R. E. Mirollo, and S. H. Strogatz, Dynamics of a Large System of Coupled Nonlinear Oscillators, Physica (Amsterdam) 52D, 293 (1991).

[118] M. C. Cross, A. Zumdieck, Ron Lifshitz, and J. L. Rogers, Synchronization by Nonlinear Frequency Pulling, Phys. Rev. Lett. 93, 224101 (2004).

[119] C. Davis-Tilley, C. K. Teoh, and A. D. Armour, Dynamics of Many-Body Quantum Synchronisation, New J. Phys. 20, 113002 (2018).

[120] J. J. García-Ripoll, S. Dürr, N. Syassen, D. M. Bauer, M. Lettner, G. Rempe, and J. I. Cirac, Dissipation-Induced Hard-Core Boson Gas in an Optical Lattice, New J. Phys. 11, 013053 (2009).

[121] B. Misra and E. C. G. Sudarshan, The Zeno's Paradox in Quantum Theory, J. Math. Phys. (N.Y.) 18, 756 (1977).

[122] A. Beige, D. Braun, B. Tregenna, and P. L. Knight, Quantum Computing Using Dissipation to Remain in a Decoherence-Free Subspace, Phys. Rev. Lett. 85, 1762 (2000).

[123] D. Rossini, A. Ghermaoui, M. B. Aguilera, R. Vatré, R. Bouganne, J. Beugnon, F. Gerbier, and L. Mazza, Strong Correlations in Lossy One-Dimensional Quantum Gases: From the Quantum Zeno Effect to the Generalized Gibbs Ensemble, arXiv:2011.04318.

[124] M. A. Lemonde, N. Didier, and A. A. Clerk, Nonlinear Interaction Effects in a Strongly Driven Optomechanical Cavity, Phys. Rev. Lett. 111, 053602 (2013). 
[125] B. A. Levitan, A. Metelmann, and A. A. Clerk, Optomechanics with Two-Phonon Driving, New J. Phys. 18, 093014 (2016).

[126] A. Kamenev, Field Theory of Non-equilibrium Systems (Cambridge University Press, Cambridge, England, 2011).

[127] H. U. R. Strand, M. Eckstein, and P. Werner, Beyond the Hubbard Bands in Strongly Correlated Lattice Bosons, Phys. Rev. A 92, 063602 (2015).

[128] H. Schoeller and G. Schön, Mesoscopic Quantum Transport: Resonant Tunneling in the Presence of a Strong Coulomb Interaction, Phys. Rev. B 50, 18436 (1994).

[129] L. Mühlbacher and E. Rabani, Real-Time Path Integral Approach to Nonequilibrium Many-Body Quantum Systems, Phys. Rev. Lett. 100, 176403 (2008).

[130] M. Schiró and M. Fabrizio, Real-Time Diagrammatic Monte Carlo for Nonequilibrium Quantum Transport, Phys. Rev. B 79, 153302 (2009).

[131] M. Schiró, Real-Time Dynamics in Quantum Impurity Models with Diagrammatic Monte Carlo, Phys. Rev. B 81, 085126 (2010).

[132] P. Werner, T. Oka, and A. J. Millis, Diagrammatic Monte Carlo Simulation of Nonequilibrium Systems, Phys. Rev. B 79, 035320 (2009).

[133] E. Gull, A. J. Millis, A. I. Lichtenstein, A. N. Rubtsov, M. Troyer, and P. Werner, Continuous-Time Monte Carlo Methods for Quantum Impurity Models, Rev. Mod. Phys. 83, 349 (2011).

[134] N. E. Bickers, Review of Techniques in the Large-N Expansion for Dilute Magnetic Alloys, Rev. Mod. Phys. 59, 845 (1987).

[135] P. Nordlander, M. Pustilnik, Y. Meir, N. S. Wingreen, and D. C. Langreth, How Long Does It Take for the Kondo Effect to Develop?, Phys. Rev. Lett. 83, 808 (1999).

[136] M. Eckstein and P. Werner, Nonequilibrium Dynamical Mean-Field Calculations Based on the Noncrossing Approximation and Its Generalizations, Phys. Rev. B 82, 115115 (2010).

[137] A. Rüegg, E. Gull, G. A. Fiete, and A. J. Millis, Sum Rule Violation in Self-Consistent Hybridization Expansions, Phys. Rev. B 87, 075124 (2013).

[138] A. Erpenbeck, E. Gull, and G. Cohen, Revealing Strong Correlations in Higher-Order Transport Statistics: A Noncrossing Approximation Approach, Phys. Rev. B 103, 125431 (2021).

[139] The spectral function obeys the frequency sum rule $\int_{-\infty}^{\infty} d \omega A(\omega)=A(t=0)=1$, which is exactly enforced by our NCA approximation of Green functions (30). This result is easily verified by using the identity $\hat{\mathcal{V}}(t=0)=\hat{\mathbb{1}}$. In the numerics, the truncation of the bosonic Hilbert space spoils this exact identity, which we therefore use to assess that the Hilbert space cutoff we consider is sufficiently large.

[140] R. Alicki, The Quantum Open System as a Model of the Heat Engine, J. Phys. A 12, L103 (1979).

[141] T. Feldmann and R. Kosloff, Quantum Four-Stroke Heat Engine: Thermodynamic Observables in a Model with Intrinsic Friction, Phys. Rev. E 68, 016101 (2003).

[142] Á. Rivas, Strong Coupling Thermodynamics of Open Quantum Systems, Phys. Rev. Lett. 124, 160601 (2020).
[143] H. E. D. Scovil and E. O. Schulz-DuBois, Three-Level Masers as Heat Engines, Phys. Rev. Lett. 2, 262 (1959).

[144] E. Boukobza and D. J. Tannor, Thermodynamic Analysis of Quantum Light Amplification, Phys. Rev. A 74, 063822 (2006).

[145] E. Boukobza and D. J. Tannor, Three-Level Systems as Amplifiers and Attenuators: A Thermodynamic Analysis, Phys. Rev. Lett. 98, 240601 (2007).

[146] Y.-Y. Liu, J. Stehlik, C. Eichler, M. J. Gullans, J. M. Taylor, and J.R. Petta, Semiconductor Double Quantum Dot Micromaser, Science 347, 285 (2015).

[147] In fact, one can show analytically, from the expression of the retarded Green function in Appendix $E$, that $\Omega_{0}$ in Hubbard-I is independent of the hopping and equivalent to the single-site and mean-field value.

[148] J. Lebreuilly, M. Wouters, and I. Carusotto, Towards Strongly Correlated Photons in Arrays of Dissipative Nonlinear Cavities under a Frequency-Dependent Incoherent Pumping, C.R. Phys. 17, 836 (2016).

[149] J. Keeling, M. H. Szymańska, and P. B. Littlewood, Keldysh Green's Function Approach to Coherence in a Non-equilibrium Steady State: Connecting Bose-Einstein Condensation and Lasing, in Optical Generation and Control of Quantum Coherence in Semiconductor Nanostructures, edited by G. Slavcheva and P. Roussignol (Springer, Berlin, 2010), pp. 293-329.

[150] A. Mitra, S. Takei, Y. B. Kim, and A. J. Millis, Nonequilibrium Quantum Criticality in Open Electronic Systems, Phys. Rev. Lett. 97, 236808 (2006).

[151] A. A. Clerk, M. H. Devoret, S. M. Girvin, . Marquardt, and R. J. Schoelkopf, Introduction to Quantum Noise, Measurement, and Amplification, Rev. Mod. Phys. 82, 1155 (2010).

[152] L. Foini, L. F. Cugliandolo, and A. Gambassi, FluctuationDissipation Relations and Critical Quenches in the Transverse Field Ising Chain, Phys. Rev. B 84, 212404 (2011).

[153] E. G. Dalla Torre, E. Demler, T. Giamarchi, and E. Altman, Dynamics and Universality in Noise-Driven Dissipative Systems, Phys. Rev. B 85, 184302 (2012).

[154] M. Schiró and A. Mitra, Transient Orthogonality Catastrophe in a Time-Dependent Nonequilibrium Environment, Phys. Rev. Lett. 112, 246401 (2014).

[155] L. Pitaevskii and S. Stringari, Bose-Einstein Condensation and Superfluidity, International series of monographs on physics (Oxford University, New York, 2016).

[156] I. S. Aranson and L. Kramer, The World of the Complex Ginzburg-Landau Equation, Rev. Mod. Phys. 74, 99 (2002).

[157] J. Keeling and S. Kéna-Cohen, Bose-Einstein Condensation of Exciton-Polaritons in Organic Microcavities, Annu. Rev. Phys. Chem. 71, 435 (2020), pMID: 32126177.

[158] T. E. Lee, H. Häffner, and M. C. Cross, Antiferromagnetic Phase Transition in a Nonequilibrium Lattice of Rydberg Atoms, Phys. Rev. A 84, 031402(R) (2011).

[159] C.-K. Chan, T. E. Lee, and S. Gopalakrishnan, Limit-Cycle Phase in Driven-Dissipative Spin Systems, Phys. Rev. A 91, 051601(R) (2015).

[160] R. M. Wilson, K. W. Mahmud, A. Hu, A. V. Gorshkov, M. Hafezi, and M. Foss-Feig, Collective Phases of Strongly 
Interacting Cavity Photons, Phys. Rev. A 94, 033801 (2016).

[161] E. T. Owen, J. Jin, D. Rossini, R. Fazio, and M. J. Hartmann, Quantum Correlations and Limit Cycles in the Driven-Dissipative Heisenberg Lattice, New J. Phys. 20, 045004 (2018).

[162] P. Degenfeld-Schonburg and M. J. Hartmann, SelfConsistent Projection Operator Theory for Quantum Many-Body Systems, Phys. Rev. B 89, 245108 (2014).

[163] M. Seclì, M. Capone, and M. Schirò, Signatures of SelfTrapping in the Driven-Dissipative Bose-Hubbard Dimer, arXiv:2102.04076.

[164] A. Strathearn, P. Kirton, D. Kilda, J. Keeling, and B. W. Lovett, Efficient Non-Markovian Quantum Dynamics Using Time-Evolving Matrix Product Operators, Nat. Commun. 9, 3322 (2018).

[165] D. Kilda and J. Keeling, Fluorescence Spectrum and Thermalization in a Driven Coupled Cavity Array, Phys. Rev. Lett. 122, 043602 (2019).

[166] M. Lotem, A. Weichselbaum, J. von Delft, and M. Goldstein, Renormalized Lindblad Driving: A Numerically Exact Nonequilibrium Quantum Impurity Solver, Phys. Rev. Research 2, 043052 (2020).

[167] E. Arrigoni, M. Knap, and W. von der Linden, Nonequilibrium Dynamical Mean-Field Theory: An Auxiliary Quantum Master Equation Approach, Phys. Rev. Lett. 110, 086403 (2013).
[168] J. Otsuki and Y. Kuramoto, Dynamical Mean-Field Theory for Quantum Spin Systems: Test of Solutions for Magnetically Ordered States, Phys. Rev. B 88, 024427 (2013).

[169] J. T. Barreiro, M. Müller, P. Schindler, D. Nigg, T. Monz, M. Chwalla, M. Hennrich, C. F. Roos, P. Zoller, and R. Blatt, An Open-System Quantum Simulator with Trapped Ions, Nature (London) 470, 486 (2011).

[170] T. E. Lee, S. Gopalakrishnan, and M. D. Lukin, Unconventional Magnetism via Optical Pumping of Interacting Spin Systems, Phys. Rev. Lett. 110, 257204 (2013).

[171] E. Haller, J. Hudson, A. Kelly, D. A. Cotta, B. Peaudecerf, G. D. Bruce, and S. Kuhr, Single-Atom Imaging of Fermions in a Quantum-Gas Microscope, Nat. Phys. 11, 738 (2015).

[172] K. Yamamoto, M. Nakagawa, K. Adachi, K. Takasan, M. Ueda, and N. Kawakami, Theory of Non-Hermitian Fermionic Superfluidity with a Complex-Valued Interaction, Phys. Rev. Lett. 123, 123601 (2019).

[173] M. Nakagawa, N. Tsuji, N. Kawakami, and M. Ueda, Dynamical Sign Reversal of Magnetic Correlations in Dissipative Hubbard Models, Phys. Rev. Lett. 124, 147203 (2020).

Correction: A proof change request to the first sentence of the abstract was not implemented properly and has now been rectified. 